\title{
Holographic models for QCD in the Veneziano limit
}

\author{
Matti Järvinen ${ }^{a}$ and Elias Kiritsis ${ }^{a, b}$ \\ ${ }^{a}$ Crete Center for Theoretical Physics, Department of Physics, University of Crete, \\ 71003 Heraklion, Greece \\ ${ }^{b}$ Laboratoire APC - AstroParticule et Cosmologie, Université Paris 7, (UMR du CNRS 7164), \\ Bâtiment Condorcet, F-75205, Paris Cedex 13, France \\ E-mail: mjarvine@physics.uoc.gr, kiritsis@physics.uoc.gr
}

ABSTRACT: We construct a class of bottom-up holographic models with physics comparable to the one expected from QCD in the Veneziano limit of large $N_{f}$ and $N_{c}$ with fixed $x=\frac{N_{f}}{N_{c}}$. The models capture the holographic dynamics of the dilaton (dual to the YM coupling) and a tachyon (dual to the chiral condensate), and are parametrized by the real parameter $x$, which can take values within the range $0 \leq x<\frac{11}{2}$. We analyze the saddle point solutions, and draw the phase diagram at zero temperature and density. The back-reaction of flavor on the glue is fully included. We find the conformal window for $x \geq x_{c}$, and the QCD-like phase with chiral symmetry breaking at $x<x_{c}$, where the critical value $x_{c}$ lies close to four. We also find Miransky scaling as $x \rightarrow x_{c}$ as well as Efimov-like saddle points. By calculating the holographic $\beta$-functions, we demonstrate the "walking" behavior of the coupling in the region near and below $x_{c}$.

KEYWORDS: Gauge-gravity correspondence, Spontaneous Symmetry Breaking, AdS-CFT Correspondence, QCD

ARXiv EPRINT: 1112.1261 


\section{Contents}

1 Introduction $\quad 1$

1.1 Bottom-up models for QCD in the quenched approximation 4

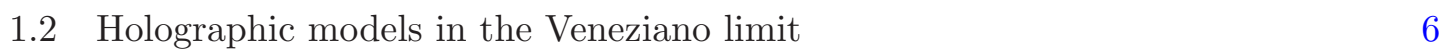

2 QCD in the Veneziano limit $\quad 10$

3 A step back: bottom-up models for large- $\mathrm{N}_{c}$ YM 12

$\begin{array}{lll}3.1 & \text { Finite temperature } & 14\end{array}$

4 Adding flavor $\quad 16$

5 The bottom-up models $\quad 19$

$\begin{array}{ll}5.1 \text { The equations of motion } & 20\end{array}$

6 Conformal fixed-point solutions $\quad 20$

7 Holographic $\boldsymbol{\beta}$-functions $\quad 21$

$\begin{array}{ll}7.1 \text { The UV fixed point } & 23\end{array}$

$\begin{array}{lll}7.2 & \text { Confining IR asymptotics } & 23\end{array}$

$\begin{array}{lll}7.3 & \text { Some simple } \beta \text { functions } & 25\end{array}$

$\begin{array}{ll}7.4 & \text { Numerical solutions for the } \beta \text { and } \gamma \text { functions }\end{array}$

$\begin{array}{lll}8 & \text { The background solutions } & \mathbf{2 7}\end{array}$

$\begin{array}{ll}\text { 8.1 Generic properties of the background } & 27\end{array}$

$\begin{array}{ll}\text { 8.2 Matching UV behavior with the QCD } \beta \text {-functions } & 29\end{array}$

8.3 Condensate dimension and the edge of the conformal window 30

\begin{tabular}{ll}
8.4 & Constructing the background solutions \\
\hline 4
\end{tabular}

8.5 Background solutions at vanishing quark mass 38

$\begin{array}{lll}\text { 8.5.1 Solution with identically vanishing tachyon } & 39\end{array}$

8.5.2 Solutions having nontrivial tachyon dependence 39

$\begin{array}{ll}8.6 & \text { Backgrounds at generic quark masses }\end{array}$

9 The free energy $\quad \mathbf{4 4}$

9.1 The free energy difference of the $m=0$ backgrounds 45

$\begin{array}{ll}10 \text { Scaling below the conformal window } & 47\end{array}$

$\begin{array}{ll}\text { 10.1 BKT scaling of the chiral condensate } & 47\end{array}$

$\begin{array}{ll}10.2 \text { Scaling of the free energy } & 50\end{array}$

10.3 Comparison with numerical results 51

11 Conclusions 
B A coordinate transformation $\quad 56$

C Examples of explicit potential choices $\quad 56$

D UV behavior $\quad \mathbf{5 9}$

$\begin{array}{lll}\text { D.1 Generic behavior } & 60\end{array}$

$\begin{array}{ll}\text { D.1.1 The UV AdS boundary at } \lambda=0 & 60\end{array}$

$\begin{array}{ll}\text { D.1.2 A bounce-back at finite } \lambda & 61\end{array}$

$\begin{array}{lll}\text { D.2 Special case: UV fixed point at finite } \lambda & 62\end{array}$

E IR behavior $\quad \mathbf{6 2}$

$\begin{array}{lll}\text { E.1 Generic cases } & 63\end{array}$

E.1.1 Divergence of the derivative of the tachyon 63

E.1.2 A bounce-back as $d \lambda / d A \rightarrow 0 \quad 63$

E.2 Special IR singularities 64

E.2.1 Generic metric singularity $\quad 64$

$\begin{array}{lll}\text { E.2.2 Special metric singularity } & 65\end{array}$

E.2.3 Tachyon behavior 66

$\begin{array}{lll}\text { E.3 Singularity at the IR fixed point with } T \equiv 0 & 69\end{array}$

$\begin{array}{ll}\text { E.3.1 Generalization to } T \ll 1 & 70\end{array}$

$\begin{array}{lll}\text { F Structure of the background as a function of } x \text { and } T_{0} & 71\end{array}$

$\begin{array}{lll}\text { F.1 Numerical analysis } & 74\end{array}$

$\begin{array}{ll}\text { G Extracting UV coefficients from numerical solutions } & \mathbf{7 7}\end{array}$

$\begin{array}{lll}\text { G.1 Extracting free energy differences } & 77\end{array}$

$\begin{array}{ll}\text { G.2 Extracting the chiral condensate at } m=0 & 77\end{array}$

$\begin{array}{ll}\text { H Details on BKT scaling } & 79\end{array}$

\section{Introduction}

Holographic techniques have been used recently in order to understand the strong dynamics of gauge theories. QCD is one of the obvious targets of such a program, but strongly coupled gauge theories may also emerge in other contexts, namely in the physics beyond the Standard model (non-perturbative electroweak symmetry breaking is an example), as well as in condensed matter contexts.

An interesting mild generalization of QCD, involves $\mathrm{SU}\left(N_{c}\right)$ YM coupled to $N_{f}$ Dirac fermions transforming in the fundamental representation (that we will still call quarks). This is a theory, that can be studied in the $\left(N_{c}, N_{f}\right)$ plane. 
As usual simplifications arise in the large- $N_{c}$ limit. The standard 't Hooft large- $N_{c}$ limit, [1], lets $N_{c} \rightarrow \infty$ keeping $N_{f}$ and $\lambda=g_{\mathrm{YM}}^{2} N_{c}$ finite. In this limit, the effect of the quarks are suppressed by powers of $\frac{N_{f}}{N_{c}} \rightarrow 0$, and therefore it corresponds to the "quenched" limit. In particular interesting dynamical effects as the conformal window, and exotic phases at finite density, driven by the presence of the quarks are not expected to be visible in the 't Hooft large- $N_{c}$ limit.

In [2] Veneziano introduced an alternative large- $N_{c}$ limit in which

$$
N_{c} \rightarrow \infty, \quad N_{f} \rightarrow \infty, \quad \frac{N_{f}}{N_{c}}=x \quad \text { fixed }, \quad \lambda=g_{\mathrm{YM}}^{2} N_{c} \quad \text { fixed }
$$

in order to make the chiral $\mathrm{U}(1)$ anomaly visible to leading order in the $1 / N_{c}$ expansion. This is the large- $N_{c}$ limit we will study in this paper.

There are several interesting issues that are accessible in the Veneziano limit.

- The "conformal window" with an IR fixed point. The window extends from $x=\frac{11}{2}$ to smaller values of $x$, and includes the Banks-Zaks (BZ) weakly-coupled region as $x \rightarrow \frac{11}{2}[3]$.

- The phase transition at a critical $x=x_{c}$ from the conformal window to theories with chiral symmetry breaking in the IR.

- A transition region near and below $x_{c}$, where the theory is expected to exhibit "walking behavior". The theory flows towards the IR fixed point but misses it ending up with chiral symmetry breaking, so that the coupling constant varies slowly over a long range of energies.

- New phenomena at finite density, involving color superconductivity [4] and flavorcolor locking [5].

The point $x=x_{c}$ is the point of a quantum phase transition where the theory passes from an IR Conformal theory (above $x_{c}$ ) to a theory with a non-trivial chiral condensate (below $x_{c}$ ). Such transitions were termed conformal phase transitions in [6], and have been recently argued to be due to the fusion of a UV and IR fixed points [7]. The scaling of the condensate is similar to that of the 2D Berezinskii-Kosterlitz-Thouless (BKT) transition [8], and is also known as Miransky scaling, [9]. Several such quantum phase transitions have been described recently in holographic theories in [10-17].

The location of the lower edge of the conformal window is determined by nonperturbative dynamics. Several estimates of the value for $x_{c}$, and for the critical value of $N_{f}$ at finite $N_{c}$ have been put forward by using different methods [18-28], and the boundary of the conformal window is also being studied actively on the lattice (see, e.g., [29-31]).

The walking region near $x_{c}$ as well as the value of $x_{c}$, have been of interest for a while, due to their potential relevance for the realization of walking technicolor, [32-34]. Technicolor has been used as a generic name for non-perturbative electroweak symmetry breaking mimicking the one induced by QCD, [35-37]. Although non-perturbative effects in a new strongly coupled gauge theory can induce electroweak symmetry breaking, generating 
the quark and lepton masses is an extra problem. A new interaction (extended technicolor) is usually invoked to generate the requisite couplings. However their magnitude (and therefore the Standard model masses) are controlled by the dimension of the scalar operator that breaks the electroweak symmetry. In free field theory its dimension is 3 , as it is a fermion bilinear. However, for the SM masses to have realistic values the dimension must be reduced to at least around 2, i.e., the anomalous dimension of the operator should be at least one. This is indeed expected to be generated by a "walking" theory [18, 19, 38]. Notice that coupling between the technicolor sector and the standard model may decrease the dimension substantially [39].

There are issues that so far have made such non-perturbative approaches to be in apparent conflict with data, like the value of the $S$ parameter, [40]. It was argued that in cases where the strongly coupled theory is near a conformal transition the $S$-parameter can be quite different potentially evading the experimental constraints, ${ }^{1}$ [44].

There have been several bottom-up models of technicolor [45-55], mostly inspired from the hard wall models for mesons, [56, 57]. Lately there have also been top-down holographic models of walking behavior, [15, 58-60]. They use several contexts like flavored MN solutions, [58], $D_{7}-\overline{D_{7}}$ pairs, [60] or $D_{3}-D_{7}$ systems, [15].

A theory that can be compared is $\mathcal{N}=1$ supersymmetric QCD with $N_{f}$ flavors. The ground states of this theory have been found by Seiberg, [61-63], and we understand several issues associated to low energy dynamics, including the Seiberg duality. Such a theory gives already several important hints on the structure expected in non-supersymmetric QCD, [64]. Defining again $x=\frac{N_{f}}{N_{c}}$, we have the following regimes:

- At $x=0$ the theory has confinement, a mass gap and $N_{c}$ distinct vacua associated with a spontaneous breaking of the leftover R symmetry $Z_{N_{c}}$.

- At $0<x<1$, the theory has a runaway ground state.

- At $x=1$, the theory has a quantum moduli space with no singularity. This reflects confinement with chiral symmetry breaking.

- At $x=1+\frac{1}{N_{c}}$, the moduli space is classical (and singular). The theory confines, but there is no chiral symmetry breaking.

- At $1+\frac{2}{N_{c}}<x<\frac{3}{2}$ the theory is in the non-abelian magnetic IR-free phase, with the magnetic gauge group $\mathrm{SU}\left(N_{f}-N_{c}\right)$ IR free.

- At $\frac{3}{2}<x<3$, the theory flows to a CFT in the IR. Near $x=3$ this is the Banks-Zaks region where the original theory has an IR fixed point at weak coupling. Moving to lower values, the coupling of the $\mathrm{IR} \mathrm{SU}\left(N_{c}\right)$ gauge theory grows. However near $x=\frac{3}{2}$ the dual magnetic $\mathrm{SU}\left(N_{f}-N_{c}\right)$ is in its Banks-Zaks region, and provides a weakly coupled description of the IR fixed point theory.

- At $x>3$, the theory is IR free.

\footnotetext{
${ }^{1}$ Recent studies that extrapolate from the Banks-Zaks region suggest that the modification of the Sparameter near the conformal window may be modest, [41-43].
} 
The region $\frac{3}{2}<x<3$ is comparable to the conformal window that is expected in (nonsupersymmetric) QCD. Indeed, the IR coupling of the original gauge theory is becoming stronger as $x$ decreases, but for $\mathrm{x}$ above $3 / 2$, a new set of IR states becomes weakly coupled, namely the magnetic gluons and quarks. These states have been interpreted as the $\rho$ mesons and their supersymmetric avatars, [65]. They are massless and weakly coupled in this region. The regime $1+\frac{2}{N_{c}}<x<\frac{3}{2}$ does not seem to have an analogue in QCD. The IR theory is again an IR non-abelian gauge theory, therefore trivially scale invariant in the far IR, but also free.

It was suggested already in [66] that the presence of a conformal window in $\mathcal{N}=1$ supersymmetric QCD is associated with the violation of the Breitenlohner-Freedman (BF) bound. In [67] a setup reminiscent of the one described here, but simpler was investigated in section 2. In particular, a non-critical $A d S_{5}$ solution was found in 5 dimensions in the presence of backreacting flavor $D_{4}-\bar{D}_{4}$ branes, and the spectrum of the open string tachyon fluctuations were analyzed as a function of $\frac{N_{f}}{N_{c}}$. However, the model was too simple to have a violation of the BF bound. A non-trivial solution was found however in 8 dimensions, using a deformation of the hermitian $K K L$ spaces, [68] generalizing the $\mathrm{N}=1$ example of [66]. A recent attempt to describe related physics was done in [69].

\subsection{Bottom-up models for QCD in the quenched approximation}

To construct a bottom-up holographic model for QCD in the Veneziano limit ${ }^{2}$ we need to first understand pure YM. The simplest bottom model for pure YM in four dimensions is the hard-wall model, first introduced in [70]. Despite its simplicity it could capture a few qualitative features of the strong interaction. More sophisticated models accounted for the running of the YM coupling constant, incorporating therefore the dilaton into the gravitational action, [71-74]. By simply adjusting a dilaton potential they could exhibit many of the properties of large- $N_{c}$ YM including confinement, a mass gap, asymptotic linear trajectories and realistic glueball spectra at zero temperature. Moreover they fared rather well at finite temperature, $[75,76]$, and after the tuning of two phenomenological parameters in the dilaton potential, ${ }^{3}[77]$, they could agree with lattice data both at zero and finite temperature, [78]. The properties of IHQCD at finite temperature were further explored in [79, 80]. Alternative Einstein-dilaton models exhibiting a cross-over rather than a first order deconfining transition, and matching YM finite temperature dynamics were also developed in [81, 82]. Such bottom up models, were used to compute transport properties of YM, like the bulk viscosity and the diffusion properties of heavy quarks, [83-85]. Backgrounds having an IR fixed point or a "walking" region, where the system flows close to an fixed point, were studied within in the IHQCD model in [28, 86-89]. In these studies the fixed point was introduced via the input $\beta$-function, without proper modeling of the dynamics of the quarks, even though $N_{f} / N_{c}$ was large.

To go beyond YM a new ingredient is needed, namely the flavor branes. An important field in this context is the order parameter for chiral symmetry breaking, dual to a complex

\footnotetext{
${ }^{2}$ We will call such a model V-QCD from now on.

${ }^{3}$ The bottom-up Einstein-dilaton model for large- $N_{c}$ YM was termed Improved Holographic QCD (IHQCD).
} 
bifundamental field $T$. In the hard wall $[56,57]$ and soft wall [90] models for mesons, such a field was added using a quadratic action, and chiral symmetry breaking proceeded by giving to such a field a vev by hand.

In [91] it was remarked that as the flavor sector of gauge theories in string theory arises from D-brane-antibrane pairs, the bifundamental field $T$ could be naturally be identified with the brane-antibrane tachyon field that had been studied profusely (around flat space) in string theory by Sen and others, [92]. The non-linear action proposed by Sen, [93] could be therefore used as a well-motivated starting point in order to study the holographic dynamics of chiral symmetry breaking. Several general features of this approach were explored in [91],

- Chiral symmetry breaking is dynamical and is induced/controlled by the tachyon Dirac-Born-Infeld (DBI) action.

- Confining asymptotics of the geometry were shown to trigger chiral symmetry breaking.

- A Gell-Mann-Oakes-Renner relation is generically satisfied.

- The Sen DBI tachyon action induces linear Regge trajectories or mesons.

- The Wess-Zumino (WZ) terms of the tachyon action, computed in string theory [94]-[96], produce the appropriate flavor anomalies, include the axial U(1) anomaly and $\eta^{\prime}$-mixing, and implement a holographic version of the ColemanWitten theorem.

In the context above, the analysis was done in the quenched approximation: the flavor sector does not backreact on the metric and dilaton. Similar results were also obtained by considering tachyon condensation in the Sakai-Sugimoto model [97-100].

In $[101,102]$ an implementation of these ideas was performed by choosing a concrete confining background, that is simple and asymptotically AdS. This was the KupersteinSonnenschein background, [103], with a constant dilaton and an $\mathrm{AdS}_{6}$ soliton. In this background the tachyon DBI action was analyzed with the following results

- The model incorporates confinement in the sense that the quark-antiquark potential computed with the usual AdS/CFT prescription confines. Moreover, magnetic quarks are screened.

- The string theory nature of the bulk fields dual to the quark bilinear currents is readily identified: they are low-lying modes living in a brane-antibrane pair.

- Chiral symmetry breaking is realized dynamically and consistently, because of the tachyon dynamics. The dynamics determines the chiral condensate uniquely as a function of the bare quark mass. It is interesting to note what the "regularity" conditions are for the tachyon that determine the vev (chiral condensate) as a function of the source (bare quark mass). By varying the condensate, the solutions for the tachyon fall into three classes: (a) a two-parameter family of solutions that start at 
the UV and where the tachyon derivative diverges at a finite radial direction (where the tachyon remains finite), (b) a two-parameter family of solutions where the tachyon remains finite in the far IR, and (c) a one parameter family of solutions, in between (a) and (b), where the tachyon diverges in the IR (and therefore reaches its ground state). Only the solutions of the family (c) are regular, and their single parameter can be mapped to the UV mass parameter.

- The mass of the $\rho$-meson grows with increasing quark mass, or, more physically, with increasing pion mass.

- By adjusting the same parameters as in $\mathrm{QCD}\left(\Lambda_{\mathrm{QCD}}, m_{u d}\right)$ a good fit can be obtained of the light meson masses.

\subsection{Holographic models in the Veneziano limit}

To construct V-QCD we will put together the experience from IHQCD and the tachyon implementation in the quenched approximation. Putting the two together we will see that, under reasonable assumptions, we obtain a phase diagram which is qualitatively in agreement with what to expect from QCD in the Veneziano limit. Moreover we will verify that changes of the bulk tachyon and dilaton potentials that are mild give the same qualitative physics. In this sense we can state confidence in our results.

The bulk action we will consider is

$$
S=S_{g}+S_{f}, \quad S_{g}=M^{3} N_{c}^{2} \int d^{5} x \sqrt{g}\left[R-\frac{4}{3} \frac{(\partial \lambda)^{2}}{\lambda^{2}}+V_{g}(\lambda)\right]
$$

with $\lambda$ the 't Hooft coupling (exponential of the dilaton $\phi$ ) and the flavor action is

$$
S_{f}=-x M^{3} N_{c}^{2} \int d^{5} x V_{f}(\lambda, T) \sqrt{\operatorname{det}\left(g_{\mu \nu}+h(\lambda) \partial_{\mu} T \partial_{\nu} T^{\dagger}\right)} .
$$

To find the vacuum (saddle point) solution we must set the gauge fields $A_{\mu}^{L, R}$ to zero, as they are not expected to have vacuum expectation values at zero density. We also take the tachyon field $T$ to be diagonal and suppressed the WZ terms as they also do not contribute to the vacuum solution.

The pure glue potential $V_{g}$ has been determined from previous studies, [77] and we will use the same here. The tachyon potential $V_{f}(\lambda, T)$ must satisfy some basic properties, that are determined by the dual theory or general properties of tachyons in string theory: (a) To provide the proper dimension for the dual operator near the boundary (b) To exponentially vanish like $\log V_{f} \sim-T^{2}+\cdots$ for $T \rightarrow \infty$. The function $h(\lambda)$ captures the transformation from the string frame to the Einstein frame in five dimensions and will be chosen appropriately.

As with IHQCD, we will arrange that the theory is logarithmically asymptotically AdS, and will implement the two-loop $\beta$-function plus one-loop anomalous dimension for the chiral condensate. Although the geometrical picture is not expected to be reliable near the boundary, the renormalization group (RG) flows that emerge are reliable at least in the IR. The UV boundary conditions we choose can be thought of as a convenient way 
of anchoring the theory in UV. We can always define a finite cutoff and evolve the theory from there in the IR.

We first analyze the fixed points of the bulk theory. Choosing a potential that implements the Banks-Zaks fixed point, its presence exists for a range of the parameter $x$. We will make choices where this is the whole range: $0<x<\frac{11}{2}$. In such a fixed point the dilaton is constant and the tachyon vanishes identically. We have also checked that choices of potential for which the fixed point exists for $x_{*}<x<\frac{11}{2}$, have qualitatively similar physics.

We define appropriate $\beta$-functions for the YM coupling and the quark mass. We then rewrite the equations following [71-73] as first order equations that specify the flow of the couplings, as well as non-linear first order equations that determine the $\beta$-functions in terms of the potentials that appear in the bulk action (1.2), (1.3).

We can calculate the dimension of the chiral condensate in the IR fixed point theory from the bulk equations. We find that it decreases monotonically with $x$ for reasonably chosen potentials. It crosses the value 2 at $x=x_{c}$ where $x_{c}$ corresponds to the end of the conformal window as argued in [7]. We make the following observations which are relevant for technicolor studies:

- The lower edge of the conformal window $x_{c}$ lies in the vicinity of 4 . Requiring the holographic $\beta$-functions to match with QCD in the UV, we find that quite in general

$$
3.7 \lesssim x_{c} \lesssim 4.2
$$

which is in good agreement with other estimates [18-28].

- The fact that the dimension of the chiral condensate at the IR fixed point approaches two (and the anomalous dimension approaches unity) as $x \rightarrow x_{c}$ is in line with the standard expectation from field theory approaches $[18,19,38]$. It is also to a large extent independent of the details of the model.

It is important to stress that in the full analysis of this paper, the backreaction of the flavor sector on the glue sector is fully included. This is very important for the "walking" region, in the vicinity of $x=4$, where we expect the backreaction to be important. Indeed we do not expect to see a Conformal Phase Transition in the quenched limit of QCD.

Apart from $x$, there is a single parameter in the theory, namely $\frac{m}{\Lambda_{\mathrm{QCD}}}$ where $m$ is the UV value of the (common) quark mass. For each value of $x$, we solve the bulk equations with fixed sources corresponding to fixed $m, \Lambda_{\mathrm{QCD}}$, and determine the vevs so that the solution is "regular" in the IR. The notion of regularity is tricky even in the case of IHQCD (pure glue), as there is a naked singularity in the far IR. For the dilaton this has been resolved in $[71-73,76]$. For the tachyon the notion of regularity is different and has been studied in detail in [101, 102].

Implementing the regularity condition in the IR and solving the equations from the IR to the UV (this has been done mostly numerically), there is a single parameter that determines the solutions as well as the UV coupling constants and vevs, and this is the a real number $T_{0}$ controlling the value of the Tachyon in the IR. This reflects the single dimensionless parameter $\frac{m}{\Lambda_{\mathrm{QCD}}}$ of the theory.

For different values of $x$ and $m$ we find the following qualitatively different regions: 
- When $x_{c} \leq x<11 / 2$ and $m=0$, the theory flows to an IR fixed point. The IR CFT is weakly coupled near $x=\frac{11}{2}$ and strongly coupled in the vicinity of $x_{c}$. Chiral symmetry is unbroken in this regime (this is known as the conformal window).

- When $x_{c} \leq x<11 / 2$ and $m \neq 0$, the tachyon has a non-trivial profile, and there is a single solution with the given source, which is "regular" in the IR.

- When $0<x<x_{c}$ and $m=0$, there is an infinite number of regular solutions with non-trivial tachyon profile, and a special solution with an identically vanishing tachyon and an IR fixed point.

- When $0<x<x_{c}$ and $m \neq 0$, the theory has vacua with nontrivial profile for the tachyon. For every non-zero $m$, there is a finite number of regular solutions that grows as $m$ approaches zero.

In the region $x<x_{c}$ where several solutions exist, there is a interesting relation between the IR value $T_{0}$ controlling the regular solutions, and the UV parameters, namely $m$. This is determined numerically, and a relevant plot describing the relation between $m$ and $T_{0}$ at fixed $x$ is in figure 6 (left). As $m$ and $-m$ are related by a chiral rotation by $\pi$, we can take $m \geq 0$.

The solutions are characterized by the number of times $n$ the tachyon field changes sign as it evolves from the UV to the IR. For all values of $m$ there is a single solution with no tachyon zeroes. In addition, for each positive $n$ there are two solutions which exist within a finite range $0<m<m_{n}$, where the limiting value $m_{n}$ decreases with increasing $n$, and one solution for $m=0$. In particular, for large enough fixed $m$, we find that only the solution without tachyon zeroes exists.

For $m \neq 0$, out of all regular solutions, the "first" one without tachyon zeroes has the smallest free energy. The same is true for $m=0$, namely the solution with non-trivial tachyon without zeroes is energetically favored over the solutions with positive $n$ as well as over the special solution with identically vanishing tachyon, which appears only for $m=0$ and would leave chiral symmetry unbroken. Therefore, chiral symmetry is broken for $x<x_{c}$.

The multiplicity of regular solutions is closely related to the regime where the IR dimension of the chiral condensate is smaller than 2, and the associated Efimov vacua. They seem to be associated with the fixed point theory that here exists for all values of $x$ but is not reachable by flowing from the UV of QCD for $x<x_{c}$. On the other hand, the presence of a fixed-point theory in the landscape of possible theories does not seem necessary for the appearance of multiple saddle points. Indeed, in [101, 102] which employed the quenched approximation and where no such fixed points exist, a second saddle point was found that provided a regular tachyon solution. It was verified however that this second saddle point was perturbatively unstable as meson fluctuations were tachyonic.

In the region just below $x_{c}$ we find Miransky or BKT scaling for the chiral condensate. As $x \rightarrow x_{c}$, we obtain

$$
\sigma \sim \Lambda_{\mathrm{QCD}}^{3} \exp \left(-\frac{2 \hat{K}}{\sqrt{x_{c}-x}}\right) .
$$


For $x \geq x_{c}$, let $m_{\mathrm{IR}}(x)$ be the mass of the tachyon at the IR fixed point and $\ell_{\mathrm{IR}}(x)$ the IR AdS radius. The coefficient $\hat{K}$ is then fixed as

$$
\hat{K}=\frac{\pi}{\sqrt{\frac{d}{d x}\left[m_{\mathrm{IR}}^{2} \ell_{\mathrm{IR}}^{2}\right]_{x=x_{c}}}} .
$$

We stress that the behavior at and below the BKT transition is to a large extent independent of the details of the model. In particular, we do not need any information on the nonlinear terms in the tachyon EoM and on how the IR boundary conditions are fixed.

The construction of the holographic V-QCD model opens the road for addressing several interesting questions.

1. The calculation of the spectrum of mesons and glueballs. This is in principle a straightforward albeit tedious exercise, [104]. In the Veneziano limit, mixing is expected between glueballs and mesons to leading order in $1 / N_{c}$. This will affect the $0^{++}$glueball that will mix with the $0^{++}$flavor-singlet $\sigma$-mesons. On the other hand the $2^{++}$glueballs, the $1^{--}$and $1^{++}$vector mesons and the $0^{+-}$mesons do not mix, with the exception of the flavor singlet $0^{+-}$meson (analogous to $\eta^{\prime}$ ) that will mix with the $0^{+-}$glueball due to the axial anomaly. A particularly interesting question here is the behavior of the mass of the lightest $0^{++}$state (the technidilaton, [105]) as $x \rightarrow x_{c}$.

2. The structure and phase diagram of the theory at finite temperature, [106]. In the quenched approximation $[101,102]$ the restoration of chiral symmetry was seen above the (first order) deconfinement transition. The expected structure is not clear here and several options exist.

3. The calculation of the energy loss of heavy quarks in a quark-gluon plasma with non-negligible percentage of quarks.

4. The construction of the baryon states in this theory and the calculation of their properties.

5. The structure of the phase diagram at finite density and the search for exotic phases namely color superconductivity and color-flavor locking.

It is plausible that the setup may provide a model for high- $T_{c}$ superconductors by interpreting the $x$ parameter as a "doping" parameter. The reason is that $x$ controls the IR dimension of the "Cooper pair" associated with a quark-antiquark boundstate, charged under the axial charge. As $x$ decreases, the IR dimension of this operator decreases, and the bound state becomes more and more deeply bound. At $x=x_{c}$, there is an onset of "axial" superconductivity (at zero temperature), that persists down to $x=0$.

At finite temperature, this picture suggests that the system might resemble the overdoped regime of strange metals, with $x=x_{c}$ the start of the superconduction dome and $x=0$ the optimal doping. The connection between the value of $x$ and doping in real systems may not be so far fetched as the changes in the system associated with the change of carriers, is accompanied by a change in the effective number of flavors of strongly interacting effective degrees of freedom. 
The structure of this paper is as follows. In section 2 we give a brief review on QCD in the Veneziano limit and its phase structure. In section 3 we review the IHQCD model, and discuss the earlier results on mass spectra and the phase structure at finite temperature. Adding the flavor branes is discussed in detail in section 4. The V-QCD model is finally introduced in section 5. Analysis of the model is started by studying the fixed points in section 6. We go on transforming the equations of motion (EoMs) to equations for the holographic $\beta$-functions, and discuss their UV/IR asymptotics and solutions in section 7 . In section 8 we analyze the background, in particular how the UV expansions map to perturbation theory of QCD, and where the edge of the conformal window appears. We also construct and present the numerical solutions for the background in the physically interesting regions. In section 9 we find the vacua with lowest free energy and check that they support the expected phase diagram. In section 10 we study the system near but below the conformal window, and show that the chiral condensate, as well as many other observables, obey the BKT scaling law. In particular, we check the scaling by comparing numerical results to formulas, that are derived analytically. Finally, we conclude and summarize the main results in section 11. Technical details are presented in appendices A-H.

\section{QCD in the Veneziano limit}

The conventional large- $N_{c}$ limit of QCD involves a large number of colors $N_{c} \rightarrow \infty$, but a fixed number of flavors, $N_{f} \rightarrow$ finite, [1]. In this limit, fermion loops are suppressed, and the dominant diagrams are classified by the genus of the associated Riemann surface. Fundamentals (quarks) are associated with open strings and boundaries, and the number of flavors is measuring the Chan-Paton factors of the open strings.

There is an alternative large- $N_{c}$ in QCD, in which

$$
N_{c} \rightarrow \infty, \quad N_{f} \rightarrow \infty, \quad \frac{N_{f}}{N_{c}}=x \quad \text { fixed }
$$

This was first introduced by Veneziano in [2] in order to have the QCD axial anomaly, of order $\mathcal{O}\left(N_{c} N_{f}\right)$ appear in the leading order in the large- $N_{c}$ expansion.

This alternative large- $N_{c}$ limit is very interesting in order to preserve important effects due to quarks. In the conventional 't Hooft limit such effects are subleading, and this is known as the quenched limit for flavor. Many efforts have been made in the last few years to consider unquenched flavor, in order to estimate the contribution of quarks to the physics of the quark-gluon plasma. Such efforts are summarized in the recent review, [107].

The following effects are not easily visible in the conventional 't Hooft limit:

- The "conformal window" with a non-trivial fixed point, that extends from $x=\frac{11}{2}$ to smaller values of $x$. The region $x \rightarrow \frac{11}{2}$ has an IR fixed point while the theory is still weakly coupled, as was analyzed by Banks and Zaks, [3].

- It is expected that at critical $x_{c}$, the conformal window will end, and for $x<x_{c}$, the theory will exhibit chiral symmetry breaking in the IR. This behavior is expected to persist down to $x=0$. Above $x>x_{c}$ the IR theory is CFT, at strong coupling that progressively becomes weak as $x \rightarrow \frac{11}{2}$. 
- Near and below $x_{c}$, there is the transition region to conventional QCD IR behavior. In this region the theory is expected to be "walking", so that the theory flows towards the IR fixed point but misses it ending up with chiral symmetry breaking. But the approach to the fixed point involves a slow variation of the YM coupling constant for a long range of energies. This has been correlated with a nontrivial dimension for the quark mass operator near two, rather than three (the free field value).

- The existence of this "walking" region makes the theory extremely interesting for applications to strong-couplings solutions to the hierarchy problem (technicolor).

- New phenomena are expected to appear at finite density driven by strong coupling and the presence of quarks. These involve color superconductivity [4] and flavor-color locking [5].

To discuss the structure expected as a function of the finite ratio $x$, defined in (2.1) we write the two-loop QCD $\beta$-function. With $N_{f}$ (non-chiral) flavors in the fundamental, the $\beta$-function reads

$$
\beta(g)=-\frac{g^{3}}{(4 \pi)^{2}}\left\{\frac{11}{3} N_{c}-\frac{2}{3} N_{f}\right\}-\frac{g^{5}}{(4 \pi)^{4}}\left\{\frac{34}{3} N_{c}^{2}-\frac{N_{f}}{N_{c}}\left[\frac{13}{3} N_{c}^{2}-1\right]\right\}+\cdots
$$

Using the 't Hooft coupling, and setting $\frac{N_{f}}{N_{c}} \rightarrow x$ we obtain

$$
\lambda \equiv g^{2} N_{c}, \quad \dot{\lambda}=-b_{0} \lambda^{2}+b_{1} \lambda^{3}+\mathcal{O}\left(\lambda^{4}\right)
$$

with

$$
b_{0}=\frac{2}{3} \frac{(11-2 x)}{(4 \pi)^{2}}, \quad \frac{b_{1}}{b_{0}^{2}}=-\frac{3}{2} \frac{(34-13 x)}{(11-2 x)^{2}} .
$$

The Banks-Zaks region is $x=11 / 2-\epsilon$ with $\epsilon \ll 1$ and positive, [3]. We obtain a fixed point of the $\beta$-function at

$$
\lambda_{*}=\frac{(8 \pi)^{2}}{75} \epsilon
$$

which is trustable in perturbation theory, as $\lambda_{*}$ can be made arbitrarily small.

The infrared fixed point has properties that are computable in perturbation theory. In particular the low-lying operators consist of the conserved stress tensor, $\operatorname{Tr}\left[F^{2}\right]$ that is now slightly irrelevant, and the $L, R$ currents that are still conserved with the exception of the $\mathrm{U}(1)$ axial current that its conservation is broken by the anomaly.

The mass operator, $\bar{\psi}_{L} \psi_{R}$ has now dimension slightly smaller than three, as attested by its perturbative anomalous dimension

$$
\begin{aligned}
-\frac{d \log m}{d \log \mu} & \equiv \gamma=\frac{a_{0}}{4 \pi} g^{2}+\frac{a_{1}}{(4 \pi)^{2}} g^{4}+\cdots \\
a_{0}=\frac{3}{4 \pi} \frac{N_{c}^{2}-1}{N_{c}}, \quad a_{1} & =\frac{1}{2(4 \pi)^{2}}\left[3 \frac{\left(N_{c}^{2}-1\right)^{2}}{2 N_{c}^{2}}-\frac{10}{3} \frac{N_{c}^{2}-1}{N_{c}} N_{f}+\frac{97}{3}\left(N_{c}^{2}-1\right)\right] .
\end{aligned}
$$

At large $N_{c}$ this becomes

$$
\gamma \simeq \frac{3}{(4 \pi)^{2}} \lambda+\frac{(203-10 x)}{12(4 \pi)^{4}} \lambda^{2}+\mathcal{O}\left(\lambda^{3}, N_{c}^{-2}\right) .
$$


One can still perturb this theory by the $\mathrm{U}\left(N_{c}\right)$-invariant mass operator (assuming all quarks have the same mass), and the theory is expected now to flow to the trivial (QCDlike) theory in the IR.

It is believed that there is also a value $x_{c}$ with $0<x_{c}<\frac{11}{2}$ so that for $x<x_{c}$ the theory flows to a trivial theory (with a mass gap) in the IR, with chiral symmetry breaking and physics isomorphic to that of standard YM. For $\frac{11}{2}>x>x_{c}$, the theory is expected to flow to a non-trivial IR fixed point, and chiral symmetry to remain unbroken, as happens in the BZ region. Generically, the IR theory is strongly coupled except in the region $x \rightarrow \frac{11}{2}$ where the fixed point theory is weakly coupled (Banks-Zaks fixed point). For $x>\frac{11}{2}$ the theory is IR free.

\section{A step back: bottom-up models for large- $\mathrm{N}_{c} \mathrm{YM}$}

The holographic dual of large $N_{c}$ Yang-Mills theory, proposed in [71-73], is based on a five-dimensional Einstein-dilaton model, with the action: ${ }^{4}$

$$
S_{5}=-M_{p}^{3} N_{c}^{2} \int d^{5} x \sqrt{g}\left[R-\frac{4}{3}(\partial \Phi)^{2}+V(\Phi)\right]+2 M_{p}^{3} N_{c}^{2} \int_{\partial M} d^{4} x \sqrt{h} K .
$$

Here, $M_{p}$ is the five-dimensional Planck scale and $N_{c}$ is the number of colors. The last term is the Gibbons-Hawking term, with $K$ being the extrinsic curvature of the boundary. The effective five-dimensional Newton constant is $G_{5}=1 /\left(16 \pi M_{p}^{3} N_{c}^{2}\right)$, and it is small in the large- $N_{c}$ limit.

Of the $5 \mathrm{D}$ coordinates $\left\{x_{i}, r\right\}_{i=0 \ldots 3}, x_{i}$ are identified with the $4 \mathrm{D}$ space-time coordinates, whereas the radial coordinate $r$ roughly corresponds to the $4 \mathrm{D}$ RG scale. We identify $\lambda \equiv e^{\Phi}$ with the running 't Hooft coupling $\lambda_{t} \equiv N_{c} g_{\mathrm{YM}}^{2}$, up to an a priori unknown multiplicative factor, ${ }^{5} \lambda=\kappa \lambda_{t}$.

The dynamics is encoded in the dilaton potential, ${ }^{6} V(\lambda)$. The small- $\lambda$ and large$\lambda$ asymptotics of $V(\lambda)$ determine the solution in the UV and the IR of the geometry respectively. For a detailed but concise description of the UV and IR properties of the solutions the reader is referred to section 2 of [76]. Here we will only mention the most relevant information:

1. For small $\lambda, V(\lambda)$ is required to have a power-law expansion of the form:

$$
V(\lambda) \sim \frac{12}{\ell^{2}}\left(1+v_{1} \lambda+v_{2} \lambda^{2}+\ldots\right), \quad \lambda \rightarrow 0 .
$$

The value at $\lambda=0$ is constrained to be finite and positive, and sets the UV AdS scale

$\ell$. The coefficients of the other terms in the expansion fix the $\beta$-function coefficients

\footnotetext{
${ }^{4}$ Similar models of Einstein-dilaton gravity were proposed independently in $[81,82]$ to describe the finite temperature physics of large $N_{c}$ YM. They differ in the UV as the dilaton corresponds to a relevant operator instead of the marginal case we study here. The gauge coupling $e^{\Phi}$ also asymptotes to a constant instead of zero in such models.

${ }^{5}$ This relation is well motivated in the UV, although it may be modified at strong coupling (see [71-73]). The quantities we will calculate do not depend on the explicit relation between $\lambda$ and $\lambda_{t}$.

${ }^{6}$ With a slight abuse of notation we will denote $V(\lambda)$ the function $V(\Phi)$ expressed as a function of $\lambda \equiv e^{\Phi}$.
} 


\begin{tabular}{|c|c|c|c|}
\hline & IHQCD & $N_{c}=3$ & $N_{c}=\infty$ \\
\hline \hline$m_{0^{*++}} / m_{0^{++}}$ & 1.61 & $1.56(11)$ & $1.90(17)$ \\
\hline$m_{2^{++}} / m_{0^{++}}$ & 1.36 & $1.40(4)$ & $1.46(11)$ \\
\hline
\end{tabular}

Table 1. Glueball masses.

for the running coupling $\lambda(E)$. If we identify the energy scale with the metric scale factor in the Einstein frame, denoted by $e^{A}$ below, we obtain [71-73]:

$$
\beta(\lambda) \equiv \frac{d \lambda}{d \log E}=-b_{0} \lambda^{2}+b_{1} \lambda^{3}+\ldots, \quad b_{0}=\frac{9}{8} v_{1}, \quad b_{1}=-\frac{9}{4} v_{2}+\frac{207}{256} v_{1}^{2} .
$$

2. For large $\lambda$, confinement and the absence of bad singularities ${ }^{7}$ require:

$$
V(\lambda) \sim \lambda^{2 Q}(\log \lambda)^{P} \quad \lambda \rightarrow \infty, \quad\left\{\begin{array}{l}
2 / 3<Q<2 \sqrt{2} / 3, \quad P \text { arbitrary } \\
Q=2 / 3, \quad P \geq 0
\end{array}\right.
$$

In particular, the values $Q=2 / 3, P=1 / 2$ reproduce an asymptotically-linear glueball spectrum, $m_{n}^{2} \sim n$, besides confinement. We will restrict ourselves to this case in what follows.

In [71-73], the single phenomenological parameters of the potential was fixed by looking at the zero-temperature spectrum, i.e. by computing various glueball mass ratios and comparing them to the corresponding lattice results. The masses are computed by deriving the effective action for the quadratic fluctuations around the background, [110] and subsequently reducing the dynamics to four dimensions.

The glueball spectrum is obtained holographically as the spectrum of normalizable fluctuations around the zero-temperature background. In IHQCD the relevant fields are the 5D metric, one scalar field (the dilaton), and one pseudoscalar field (the axion that is subleading in $N_{c}$ ). As a consequence, the only normalizable fluctuations above the vacuum correspond to spin 0 and spin 2 glueballs (more precisely, states with $J^{P C}=$ $\left.0^{++}, 0^{-+}, 2^{++}\right)$, each species containing an infinite discrete tower of excited states.

We only compare the mass spectrum obtained in our model to the lattice results for the lowest $0^{++}, 0^{-+}, 2^{++}$glueballs and their available excited states. These are limited to one for each spin 0 species, and none for the spin 2, in the study of $[111,112]$, which is the one we use for our comparison. This provides two mass ratios in the CP-even sector and two in the CP-odd sector.

The glueball masses are computed by first solving numerically Einstein's equations, and using the resulting metric and dilaton to setup an analogous Schrödinger problem for the fluctuations, [71-73]. The results for the parity-conserving sector are shown in table 1, and are in good agreement with lattice data for $N_{c}=3$.

\footnotetext{
${ }^{7}$ For a description of the notion of "bad versus good singularities" and their resolution the reader is referred to [108, 109].
} 
Unlike the various mass ratios, the value of any given mass in AdS-length units (e.g. $m_{0++} \ell$ ) does depend on the choice of integration constants in the UV. Therefore its numerical value does not have an intrinsic meaning. However it can be used as a benchmark against which all other dimension-full quantities can be measured (provided one always uses the same UV boundary conditions). On the other hand, given a fixed set of initial conditions, asking that $m_{0++}$ matches the physical value (in $\mathrm{MeV}$ ) obtained on the lattice, fixes the value of $\ell$ hence the energy unit.

The holographic renormalization of such Einstein-dilaton theories is quite intricate as the AdS boundary conditions on the dilaton is unusual ( $\phi \rightarrow-\infty$ near the boundary). It has been derived recently in [113].

\subsection{Finite temperature}

In the large $N_{c}$ limit, the canonical ensemble partition function of the model just described, can be approximated by a sum over saddle points, each given by a classical solution of the Einstein-dilaton field equations:

$$
\mathcal{Z}(\beta) \simeq e^{-\mathcal{S}_{1}(\beta)}+e^{-\mathcal{S}_{2}(\beta)}+\ldots
$$

where $\mathcal{S}_{i}$ are the euclidean actions evaluated on each classical solution with a fixed temperature $T=1 / \beta$, i.e. with euclidean time compactified on a circle of length $\beta$. There are two possible types of Euclidean solutions which preserve 3-dimensional rotational invariance. In conformal coordinates these are:

1. Thermal gas solution,

$$
d s^{2}=b_{o}^{2}(r)\left(d r^{2}+d t^{2}+d x_{m} d x^{m}\right), \quad \Phi=\Phi_{o}(r),
$$

with $r \in(0, \infty)$ for the values of $P$ and $Q$ we are using;

2. Black-hole solutions,

$$
d s^{2}=b(r)^{2}\left[\frac{d r^{2}}{f(r)}+f(r) d t^{2}+d x_{m} d x^{m}\right], \quad \Phi=\Phi(r),
$$

with $r \in\left(0, r_{h}\right)$, such that $f(0)=1$, and $f\left(r_{h}\right)=0$.

In both cases Euclidean time is periodic with period $\beta_{o}$ and $\beta$ respectively for the thermal gas and black-hole solution, and 3 -space is taken to be a torus with volume $V_{3 o}$ and $V_{3}$ respectively, so that the black-hole mass and entropy are finite. ${ }^{8}$

The black holes are dual to a deconfined phase, since the string tension vanishes at the horizon, and the Polyakov loop has non-vanishing expectation value. On the other hand, the thermal gas background is confining.

\footnotetext{
${ }^{8}$ The periods and 3 -space volumes of the thermal gas solution are related to the black-hole solution values by requiring that the geometry of the two solutions are the same on the (regulated) boundary. See [76] for details.
} 
The thermodynamics of the deconfined phase is dual to the 5D black-hole thermodynamics. The free energy, defined as

$$
\mathcal{F}=E-T S,
$$

is identified with the black-hole on-shell action; as usual, the energy $E$ and entropy $S$ are identified with the black-hole mass, and one fourth of the horizon area in Planck units, respectively.

The thermal gas and black-hole solutions with the same temperature differ at $O\left(r^{4}\right)$ :

$$
b(r)=b_{o}(r)\left[1+\mathcal{G} \frac{r^{4}}{\ell^{3}}+\ldots\right], \quad f(r)=1-\frac{C}{4} \frac{r^{4}}{\ell^{3}}+\ldots \quad r \rightarrow 0,
$$

where $\mathcal{G}$ and $C$ are constants with units of energy. As shown in [76] they are related to the enthalpy $T S$ and the gluon condensate $\left\langle\operatorname{tr} F^{2}\right\rangle$ :

$$
C=\frac{T S}{M_{p}^{3} N_{c}^{2} V_{3}}, \quad \mathcal{G}=\frac{22}{3(4 \pi)^{2}} \frac{\left\langle\operatorname{tr} F^{2}\right\rangle_{T}-\left\langle\operatorname{tr} F^{2}\right\rangle_{o}}{240 M_{p}^{3} N_{c}^{2}} .
$$

Although they appear as coefficients in the UV expansion, $C$ and $\mathcal{G}$ are determined by regularity at the black-hole horizon. For $T$ and $S$ the relation is the usual one,

$$
T=-\frac{\dot{f}\left(r_{h}\right)}{4 \pi}, \quad S=\frac{\text { Area }}{4 G_{5}}=4 \pi\left(M_{p}^{3} N_{c}^{2} V_{3}\right) b^{3}\left(r_{h}\right) .
$$

For $\mathcal{G}$ the relation with the horizon quantities is more complicated and cannot be put in a simple analytic form. However, as discussed in [75], for each temperature there exist only specific values of $\mathcal{G}$ (each corresponding to a different black hole) such that the horizon is regular.

At any given temperature there can be one or more solutions: the thermal gas is always present, and there can be different black holes with the same temperature. The solution that dominates the partition function at a certain $T$ is the one with smallest free energy. The free energy difference between the black hole and thermal gas was calculated in [75] to be:

$$
\frac{\mathcal{F}}{M_{p}^{3} N_{c}^{2} V_{3}}=\frac{\mathcal{F}_{\mathrm{BH}}-\mathcal{F}_{\mathrm{th}}}{M_{p}^{3} N_{c}^{2} V_{3}}=15 \mathcal{G}-\frac{C}{4}
$$

For a dilaton potential corresponding to a confining theory, like the one we will assume, the phase structure is the following [75]:

1. There exists a minimum temperature $T_{\min }$ below which the only solution is the thermal gas.

2. Two branches of black holes ("large" and "small") appear for $T \geq T_{\min }$, but the ensemble is still dominated by the confined phase up to a temperature $T_{c}>T_{\min }$

3. At $T=T_{c}$ there is a first order phase transition to the large black-hole phase. The system remains in the black-hole (deconfined) phase for all $T>T_{c}$.

The holographic mode has also been confronted successfully with recent lattice data [78] at finite temperature, [77]. 


\section{Adding flavor}

A number $N_{f}$ of quark flavors can be included in our setup by adding space-time filling "flavor-branes". In this case they are pairs of space-filling $D_{4}-\overline{D_{4}}$ branes.

To motivate the setup it is important to revisit the low-dimension operators (dimension=3) in the flavor sector and their realization in string theory. At the spin-zero level we have the (complex) mass operator

$$
\bar{\psi}_{R}^{i} \psi_{L}^{j} \leftrightarrow T_{i j}
$$

dual to a complex scalar transforming as $\left(N_{f}, \bar{N}_{f}\right)$ under the flavor symmetry $\mathrm{U}\left(N_{f}\right)_{R} \times$ $\mathrm{U}\left(N_{f}\right)_{L}$. At the spin-one level we have the two classically conserved currents

$$
\bar{\psi}_{L}^{i} \sigma^{\mu} \psi_{L}^{j} \leftrightarrow A_{L, i j}^{\mu}, \quad \bar{\psi}_{R}^{i} \bar{\sigma}^{\mu} \psi_{R}^{j} \leftrightarrow A_{R, i j}^{\mu}
$$

They transform in the adjoint of the $\mathrm{U}\left(N_{f}\right)_{R}$ respectively the $\mathrm{U}\left(N_{f}\right)_{L}$ symmetry. The flavor symmetry is expected to arise in string theory from $N_{f}$ flavor branes (R) and $N_{f}$ flavor antibranes (L). Due to the quantum numbers, the vectors are the lowest modes of the fluctuations of the open strings with both ends on the $\mathrm{D}$ branes $\left(A_{R}^{\mu}\right)$, or the anti-D branes, $A_{L}^{\mu}$.

The bifundamental scalar $T$, on the other hand, is the lowest mode of the $D-\bar{D}$ strings, compatible with its quantum numbers. Its holographic dynamics is dual to the dynamics of the chiral condensate. This is precisely the scalar that in a brane-antibrane system in flat space is the tachyon whose dynamics has been studied profusely in string theory, [93]. It has been proposed that the non-linear DBI-like actions proposed by Sen and others are the proper setup in order to study the holographic dynamics of chiral symmetry breaking, [91]. This dynamics was analyzed in a toy example, [101, 102], improving several aspects of the hard [56, 57], and soft wall models, [90]. We will keep referring to $T$ as the "tachyon", as it indeed corresponds to a relevant operator in the UV.

The tachyon dynamics is captured holographically by the open string DBI+WZ action, which schematically reads, in the string frame,

$$
S\left[T, A^{L}, A^{R}\right]=S_{\mathrm{DBI}}+S_{\mathrm{WZ}}
$$

where the DBI action for the $D-\bar{D}$ pair is

$$
\begin{aligned}
S_{\mathrm{DBI}}= & \int d r d^{4} x \frac{N_{c}}{\lambda} \operatorname{Str}\left[V ( T ) \left(\sqrt{-\operatorname{det}\left(g_{\mu \nu}+D_{\{\mu} T^{\dagger} D_{\nu\}} T+F_{\mu \nu}^{L}\right)}+\right.\right. \\
& \left.\left.+\sqrt{-\operatorname{det}\left(g_{\mu \nu}+D_{\{\mu} T^{\dagger} D_{\nu\}} T+F_{\mu \nu}^{R}\right)}\right)\right]
\end{aligned}
$$

Here $T$ is the tachyon, a complex $N_{f} \times N_{f}$ matrix. $A_{\mu}^{L, R}$ are the world-volume gauge fields of the $\mathrm{U}\left(N_{f}\right)_{L} \times \mathrm{U}\left(N_{f}\right)_{R}$ flavor symmetry, under which the tachyon is transforming as the $\left(N_{f}, \bar{N}_{f}\right)$, a fact reflected in the presence of the covariant derivatives ${ }^{9}$

$$
D_{\mu} T \equiv \partial_{\mu} T-i T A_{\mu}^{L}+i A_{\mu}^{R} T, \quad D_{\mu} T^{\dagger} \equiv \partial_{\mu} T^{\dagger}-i A_{\mu}^{L} T^{\dagger}+i T^{\dagger} A_{\mu}^{R}
$$

\footnotetext{
${ }^{9}$ We are using the conventions of [91].
} 
transforming covariantly under

$$
T \rightarrow V_{R} T V_{L}^{\dagger}, \quad A^{L} \rightarrow V_{L}\left(A^{L}-i V_{L}^{\dagger} d V_{L}\right) V_{L}^{\dagger}, \quad A^{R} \rightarrow V_{R}\left(A^{R}-i V_{R}^{\dagger} d V_{R}\right) V_{R}^{\dagger}
$$

as well as the field strengths $F^{L, R}=d A_{L, R}-i A_{L, R} \wedge A_{L, R}$ of the $A^{L, R}$ gauge fields. $\lambda \equiv e^{\Phi}=N_{c} e^{\phi}$ is as usual the 't Hooft coupling. We have also used the symmetric trace (三Str) prescription although higher order terms of the non-abelian DBI action are not known. It turns out that such a prescription is not relevant for the vacuum structure in the flavor sector (as determined by the classical solution of the tachyon) neither for the mass spectrum. The reason is that we may treat the light quark masses as equal to the first approximation and then in the vacuum, $T=\tau \mathbf{1}$ with $\tau$ real, and this is insensitive to non-abelian ramifications. Expanding around this solution, the non-abelian ambiguities in the higher order terms do not enter at quadratic order. Therefore, for the spectrum we might as well replace $S t r \rightarrow T r$.

The WZ action on the other hand is given by: ${ }^{10}$

$$
S_{\mathrm{WZ}}=T_{4} \int_{M_{5}} C \wedge \operatorname{str} \exp \left[i 2 \pi \alpha^{\prime} \mathcal{F}\right]
$$

where $M_{5}$ is the world-volume of the $\mathrm{D}_{4}-\overline{\mathrm{D}_{4}}$ branes that coincides with the full space-time. Here, $C$ is a formal sum of the RR potentials $C=\sum_{n}(-i)^{\frac{5-n}{2}} C_{n}$, and $\mathcal{F}$ is the curvature of a superconnection $\mathcal{A}$. Note also that str in (4.7) stands for supertrace and not symmetric trace. It acts on the space of $D$ and $\bar{D}$ branes and is defined in appendix $\mathrm{C}$ of [91].

In terms of the tachyon field matrix $T$ and the gauge fields $A^{L}$ and $A^{R}$ living respectively on the branes and antibranes, they are (We will set $2 \pi \alpha^{\prime}=1$ and use the notation of $[95])$ :

$$
i \mathcal{A}=\left(\begin{array}{cc}
i A_{L} & T^{\dagger} \\
T & i A_{R}
\end{array}\right), \quad i \mathcal{F}=\left(\begin{array}{cc}
i F_{L}-T^{\dagger} T & D T^{\dagger} \\
D T & i F_{R}-T T^{\dagger}
\end{array}\right)
$$

The curvature of the superconnection is defined as:

$$
\mathcal{F}=d \mathcal{A}-i \mathcal{A} \wedge \mathcal{A}, \quad d \mathcal{F}-i \mathcal{A} \wedge \mathcal{F}+i \mathcal{F} \wedge \mathcal{A}=0
$$

Note that under (flavor) gauge transformation it transforms homogeneously

$$
\mathcal{F} \rightarrow\left(\begin{array}{cc}
V_{L} & 0 \\
0 & V_{R}
\end{array}\right) \mathcal{F}\left(\begin{array}{cc}
V_{L}^{\dagger} & 0 \\
0 & V_{R}^{\dagger}
\end{array}\right)
$$

In [91] the relevant definitions and properties of this supermatrix formalism can be found.

By expanding we obtain

$$
S_{\mathrm{WZ}}=T_{4} \int C_{5} \wedge Z_{0}+C_{3} \wedge Z_{2}+C_{1} \wedge Z_{4}+C_{-1} \wedge Z_{6}
$$

where $Z_{2 n}$ are appropriate forms coming from the expansion of the exponential of the superconnection. In particular, $Z_{0}=0$, signaling the global cancelation of 4-brane charge,

\footnotetext{
${ }^{10}$ This expression was proposed in [94] and proved in [95, 96] using boundary string field theory.
} 
which is equivalent to the cancelation of the gauge anomaly in QCD. Further, as was shown in [91]

$$
Z_{2}=d \Omega_{1}, \quad \Omega_{1}=i \operatorname{str}\left(V\left(T^{\dagger} T\right)\right) \operatorname{Tr}\left(A_{L}-A_{R}\right)-\log \operatorname{det}(T) d\left(\operatorname{Str} V\left(T^{\dagger} T\right)\right)
$$

This term provides the Stuckelberg mixing between $\operatorname{Tr}\left[A_{\mu}^{L}-A_{\mu}^{R}\right]$ and the QCD axion that is dual to $C_{3}$. Unlike the 't Hooft limit, in the Veneziano limit this mixing happens at leading order in $1 / N_{c},[2]$. Dualizing the full action we obtain

$$
\begin{aligned}
S_{\mathrm{CP}-\mathrm{odd}} & =\frac{M^{3}}{2 N_{c}^{2}} \int d^{5} x \sqrt{g} Z(\lambda)\left(\partial a+i \Omega_{1}\right)^{2} \\
& =\frac{M^{3}}{2 N_{c}^{2}} \int d^{5} x \sqrt{g} Z(\lambda)\left(\partial_{\mu} a+x \zeta \partial_{\mu} V(\tau)-x V(\tau) A_{\mu}^{A}\right)^{2}
\end{aligned}
$$

with

$$
\zeta=\frac{1}{N_{f}} \operatorname{Im} \log \operatorname{det} T, \quad A_{L}-A_{R} \equiv \frac{1}{2 N_{f}} A^{A} \mathrm{II}+\left(A_{L}^{a}-A_{R}^{a}\right) \lambda^{a}
$$

and where we have set the tachyon to its vev $T=\tau \mathbf{1}$. This term is invariant under the $\mathrm{U}(1)_{A}$ transformations

$$
\zeta \rightarrow \zeta+\epsilon, \quad A_{\mu}^{A} \rightarrow A_{\mu}^{A}-\partial_{\mu} \epsilon, \quad a \rightarrow a-x \epsilon V(\tau)
$$

reflecting the QCD U(1) $A$ anomaly. It is this Stuckelberg term together with the kinetic term of the tachyon field that is responsible for the mixing between the QCD axion and the $\eta^{\prime}$. In terms of degrees of freedom, we have two scalars $a, \zeta$ and an (axial) vector, $A_{\mu}^{A}$. We can use gauge invariance to remove the longitudinal components of $A^{A}$. Then an appropriate linear combination of the two scalars will become the $0^{-+}$glueball field while the other will be the $\eta^{\prime}$. The transverse (5D) vector will provide the tower of $\mathrm{U}(1)_{A}$ vector mesons.

The next term in the WZ expansion couples the baryon density to a one-form RR field $C_{1}$. There is no known operator expected to be dual to this bulk form. However its presence and coupling to baryon density can be understood as follows. Before decoupling the $N_{c} D_{3}$ branes, its dual form $C_{2}$ couples to the $\mathrm{U}(1)_{B}$ on the $D_{3}$ branes via the standard $C_{2} \wedge F_{B}$ WZ coupling. This is dual to a free field, the doubleton, living only at the boundary of the bulk. Once we add the probe $D_{4}+\bar{D}_{4}$ branes the free field is now a linear combination of $A^{B}$ and an $N_{f} / N_{c}$ admixture of $A^{V}$ originating on the flavor branes. The orthogonal combination is the baryon number current on the flavor branes and it naturally couples to $C_{1}$. Therefore the $C_{1}$ field is expected to be dual to the topological baryon current at the boundary.

Finally the form of the last term requires some explanation. By writing $Z_{6}=d \Omega_{5}$ we may rewrite this term as

$$
\int F_{0} \wedge \Omega_{5}, \quad F_{0}=d C_{-1}
$$

$F_{0} \sim N_{c}$ is nothing else but the dual of the five-form field strength. This term then provides the correct Chern-Simons form that reproduces the flavor anomalies of QCD. Its explicit form in terms of the gauge fields $A_{L, R}$ and the tachyon was given in equation (3.13) in [91].

The action as described is based on the flat space Sen action for the $D-\bar{D}$ braneantibrane system. In the presence of curvature and other non-trivial background fields, 
like the dilaton we expect corrections to the DBI action. Such corrections may affect the tachyon potential as well as the kinetic terms of the vectors and the tachyon.

However it should be stressed that as shown in [71-73], the glue backgrounds that are describing YM are asymptotically flat in the IR in the string frame. As the Sen action is naturally written in the string frame, the flat space results can be used to describe the asymptotics. Of course here the dilaton is running unlike the flat space case. However we expect that as it happens for the standard string effective action, higher-derivative corrections of the dilaton can be redefined away. Therefore, it is only the integrating out of the non-propagating four-form that will introduce unusual dilaton potential dependence. This is the reason that our ansatz for the tachyon action is that of Sen with dilaton dependent coefficients.

\section{The bottom-up models}

At $x=\frac{N_{f}}{N_{c}}=0$ the IHQCD model, [71-73], is described by the action

$$
S_{g}=M^{3} N_{c}^{2} \int d^{5} x \sqrt{g}\left[R-\frac{4}{3} \frac{(\partial \lambda)^{2}}{\lambda^{2}}+V_{g}(\lambda)\right]
$$

with $\lambda$ the 't Hooft coupling (exponential of the dilaton) and a potential that has the following asymptotics.

$$
\lim _{\lambda \rightarrow 0} V_{g}(\lambda)=\frac{12}{\ell^{2}}\left[1+v_{1} \lambda+v_{2} \lambda^{2}+\cdots\right], \quad \lim _{\lambda \rightarrow \infty} V_{g}(\lambda) \sim \lambda^{\frac{4}{3}} \sqrt{\log \lambda} .
$$

At finite $x$ we must add the flavor sector. For the vacuum structure, and with all masses of the quarks being equal it is enough to add the $\mathrm{U}(1)$ part of the tachyon DBI action,

$$
S_{f}=-x M^{3} N_{c}^{2} \int d^{5} x V_{f}(\lambda, T) \sqrt{\operatorname{det}\left(g_{\mu \nu}+h(\lambda, T) \partial_{\mu} T \partial_{\nu} T^{\dagger}\right)}
$$

where we have set the gauge fields to zero. The total action is $S=S_{g}+S_{f}$. Note that the overall sign of the DBI action is negative. The function $V_{g}$ and its asymptotics has been discussed in detail in [71-73]. We will consider it known, and when needed we will use the form that was in agreement with YM data, [77]. The tachyon potential $V_{f}(\lambda, T)$ should satisfy some basic principles. For flat space D-branes, $V_{s} \sim \frac{1}{\lambda} e^{-\mu^{2} T^{2}}$. In our case, near the boundary, where $T \rightarrow 0, \lambda \rightarrow 0$, we expect, in analogy with $V_{g}$, a regular series expansion in $\lambda, T$

$$
V_{f} \simeq V_{0}(\lambda)+V_{1}(\lambda) T^{2}+\mathcal{O}\left(T^{4}\right)
$$

with $V_{0,1}(\lambda)$ having regular power series expansions in $\lambda$. As we will see later, the functions $V_{0,1}(\lambda)$ may be mapped into the perturbative $\beta$-functions for the gauge coupling constant, and the anomalous dimension of the quark mass operator.

Near the condensation point, $T \rightarrow \infty$ we expect the potential $V_{f}$ to vanish exponentially. This is based on very general arguments due to Sen that guarantee that the brane gauge fields disappear beyond that point.

Finally the function $h(\lambda, T)$ was introduced to accommodate the fact that the action in (5.3) is written in the Einstein frame. In flat space, this factor is unity in the string frame but becomes nontrivial $\left(h \sim \lambda^{-\frac{4}{3}}\right)$ in the Einstein frame. 


\subsection{The equations of motion}

Collecting the action of the glue and flavor sectors together,

$$
\mathcal{L}=\left(M^{3} N_{c}^{2}\right)\left[\sqrt{-g}\left(R-\frac{4}{3} \frac{(\partial \lambda)^{2}}{\lambda^{2}}+V_{g}(\lambda)\right)-x V_{f}(\lambda, T) \sqrt{\operatorname{det}\left(g_{a b}+h(\lambda, T) \partial_{a} T \partial_{b} T\right)}\right] .
$$

We shall take the following Lorentz-invariant Ansatz for the metric:

$$
d s^{2}=e^{2 A}\left(d x_{1,3}^{2}+d r^{2}\right) .
$$

In our Ansatz the warp factor $A$, the scalar $\lambda$ and the tachyon $T$ depend only on the radial coordinate $r$.

The Einstein equations take the form:

$$
R_{a b}-\frac{1}{2} g_{a b} R=T_{a b}^{g}+T_{a b}^{f},
$$

where $T_{a b}^{g}$ and $T_{a b}^{f}$ are the energy momentum tensors of the glue and flavor sectors, respectively. These equations translate into

$$
\begin{aligned}
6 A^{\prime \prime}+6\left(A^{\prime}\right)^{2} & =-\frac{4}{3} \frac{\left(\lambda^{\prime}\right)^{2}}{\lambda^{2}}+e^{2 A} V_{g}(\lambda)-x V_{f}(\lambda, T) e^{2 A} \sqrt{1+e^{-2 A} h(\lambda, T)\left(T^{\prime}\right)^{2}} \\
12\left(A^{\prime}\right)^{2} & =\frac{4}{3} \frac{\left(\lambda^{\prime}\right)^{2}}{\lambda^{2}}+e^{2 A} V_{g}(\lambda)-x V_{f}(\lambda, T) \frac{e^{2 A}}{\sqrt{1+e^{-2 A} h(\lambda, T)\left(T^{\prime}\right)^{2}}}
\end{aligned}
$$

where primes stand for r-derivatives. For $x=0$ these equations agree with [71-73]. Finally, the equations of motion for the dilaton and the tachyon are given by:

$$
\begin{aligned}
\lambda^{\prime \prime}-\frac{\left(\lambda^{\prime}\right)^{2}}{\lambda}+3 A^{\prime} \lambda^{\prime}= & \frac{3}{8} \lambda^{2} e^{2 A}\left[-\frac{d V_{g}}{d \lambda}+x \frac{\partial V_{f}}{\partial \lambda} \sqrt{1+e^{-2 A} h(\lambda, T)\left(T^{\prime}\right)^{2}}\right. \\
+ & \left.\frac{x}{2} \frac{\partial h}{\partial \lambda} \frac{e^{-2 A} V_{f}\left(T^{\prime}\right)^{2}}{\sqrt{1+e^{-2 A} h(\lambda, T)\left(T^{\prime}\right)^{2}}}\right] ; \\
T^{\prime \prime}+e^{-2 A}\left(4 h A^{\prime}+\frac{\partial V_{f}}{\partial \lambda} \frac{h \lambda^{\prime}}{V_{f}}+\right. & \left.\frac{\lambda^{\prime}}{2} \frac{\partial h}{\partial \lambda}\right)\left(T^{\prime}\right)^{3}+\left(\frac{1}{2 h} \frac{\partial h}{\partial T}-\frac{1}{V_{f}} \frac{\partial V_{f}}{\partial T}\right)\left(T^{\prime}\right)^{2}+ \\
& +\left(3 A^{\prime}+\frac{\lambda^{\prime}}{V_{f}} \frac{\partial V_{f}}{\partial \lambda}+\frac{\lambda^{\prime}}{h} \frac{\partial h}{\partial \lambda}\right) T^{\prime}-\frac{e^{2 A}}{h V_{f}} \frac{\partial V_{f}}{\partial T}=0 .
\end{aligned}
$$

\section{Conformal fixed-point solutions}

There are solutions to the equations above that are conformal, and are related to the fixed points of an "effective potential". To find them we must set $\lambda^{\prime}=T^{\prime}=0$, and $\lambda^{\prime \prime}=T^{\prime \prime}=0$ in the equations (5.10), (5.11) which imply that we must be at a critical point of the effective potential,

$$
V_{\mathrm{eff}}=V_{g}(\lambda)-x V_{f}(\lambda, T), \quad \partial_{T} V_{\mathrm{eff}}=\partial_{\lambda} V_{\mathrm{eff}}=0 .
$$


For any solution $\lambda_{*}, T_{*}$ of the above conditions, we obtain an $\mathrm{AdS}_{5}$ space with

$$
\frac{12}{\ell^{2}}=V_{\mathrm{eff}}\left(\lambda_{*}, T_{*}\right)
$$

as is obvious from equations (5.8) and (5.9).

When $V_{f}$ has the standard dependence on the tachyon [93], there are two solutions to the condition $\partial_{T} V_{\text {eff }}=0$, namely $T=0$ (chiral symmetry unbroken) and $T \rightarrow \infty$, (chiral symmetry broken).

- $T=0$. In this case the second condition of extremality $(6.1)$ is $\partial_{\lambda}\left(V_{g}(\lambda)-x V_{f}(\lambda, 0)\right)=$ 0 . In the UV, $\lambda \rightarrow 0$, this potential is constructed to emulate the perturbative $\beta$ function, and therefore has a free-field theory fixed point. This is the UV theory. In the IR, it will also have a fixed point at $\lambda=\lambda_{*}$. In the $\mathrm{BZ}$ region $\lambda_{*} \ll 1$. A priori there are two possibilities.

1. The fixed point disappears for $x \leq x_{*}$ for a given $x_{*}$.

2. The fixed point exists for all $0<x<\frac{11}{2}$.

We will discuss these two options later on in this paper.

- $T \rightarrow \infty$. In this case as $\lim _{T \rightarrow \infty} V_{f}(\lambda, T)=0$ we obtain that the second extremality condition is $\partial_{\lambda} V_{g}=0$. This is equivalent to the existence of a fixed point in large-N YM theory. It has been argued however in [71-73] that this is not true. Therefore, there is no fixed point with $T \rightarrow \infty$.

\section{$7 \quad$ Holographic $\beta$-functions}

In holographic theories we may define non-perturbative $\beta$-functions that capture the dependence of coupling constants on the RG scale. This concept was developed and used first in [71-73], in order to explore the physics of Einstein dilaton theories and their holographic relation to YM theory. In particular in [71-73] it was shown that the $\beta$ function is intimately related to the generalized superpotential. It was shown later in [76] that such defined $\beta$-functions are indeed related to the quantum breaking of scale invariance, and appear in the trace of the stress-tensor. Such relations were shown in full generality in [113] and have been confirmed also in [114].

To define the $\beta$-functions we need a notion of energy scale. At the two-derivative level, such a function is the scale factor $e^{A}$, and indeed near the AdS boundary it can be identified as the energy scale. It remains always a decreasing function, and becomes zero in the ultimate IR. It can therefore be taken as the energy scale in the whole of the bulk space.

We therefore define the $\beta$-function and "anomalous" dimension $\gamma \operatorname{as}^{11}$

$$
\frac{d \lambda}{d A} \equiv \beta(\lambda, T), \quad \frac{d T}{d A} \equiv \gamma(\lambda, T) .
$$

\footnotetext{
${ }^{11}$ Notice that, as we shall see later, it is the ratio $\gamma(\lambda, T) / T$ (rather than $\gamma(\lambda, T)$ ) which corresponds closely to the anomalous dimension of the quark mass in QCD. Excluding the extra $T$ in the definition simplifies many of the equations below.
} 
The equations of motion provide equations for the $\beta$-functions. To obtain them we must convert radial derivatives to derivatives with respect to $A$,

$$
\lambda^{\prime}=A^{\prime} \beta, \quad T^{\prime}=A^{\prime} \gamma, \quad \lambda^{\prime \prime}=\beta A^{\prime \prime}+\left(\gamma \beta_{T}+\beta \beta_{\lambda}\right) A^{\prime 2}, \quad T^{\prime \prime}=\gamma A^{\prime \prime}+\left(\gamma \gamma_{T}+\beta \gamma_{\lambda}\right) A^{2}
$$

where $\beta_{T} \equiv \frac{\partial \beta}{\partial T}$ etc. Substituting in (5.9) we obtain

$$
12\left(1-\frac{\beta^{2}}{9 \lambda^{2}}\right) \frac{A^{\prime 2}}{e^{2 A}}=V_{g}-\frac{x V_{f}}{\sqrt{1+h \gamma^{2} \frac{A^{\prime 2}}{e^{2 A}}}}
$$

which can in principle be solved for $\frac{A^{\prime 2}}{e^{2 A}}$ as a function of $\lambda, V_{g}, V_{f}, h, \beta, \gamma$. In general, the solution is not unique. ${ }^{12}$ Similarly from (5.8) we obtain

$$
\frac{A^{\prime \prime}}{e^{2 A}}=-\left(1+\frac{2 \beta^{2}}{9 \lambda^{2}}\right) \frac{A^{\prime 2}}{e^{2 A}}+\frac{V_{g}-x V_{f} \sqrt{1+h \gamma^{2} \frac{A^{\prime 2}}{e^{2 A}}}}{6} .
$$

Then (5.10) and (5.11) become

$$
\begin{aligned}
& \left(\gamma \frac{\partial \beta}{\partial T}+\beta \frac{\partial \beta}{\partial \lambda}-\frac{\beta^{2}}{\lambda}\right) \frac{A^{\prime 2}}{e^{2 A}}+\frac{\beta}{6}\left(2 V_{g}-\frac{x V_{f}}{\sqrt{1+h \gamma^{2} \frac{A^{\prime 2}}{e^{2 A}}}}-x V_{f} \sqrt{1+h \gamma^{2} \frac{A^{\prime 2}}{e^{2 A}}}\right) \\
& =\frac{3}{8} \lambda^{2}\left[-\frac{\partial V_{g}}{\partial \lambda}+x \frac{\partial V_{f}}{\partial \lambda} \sqrt{1+h \gamma^{2} \frac{A^{\prime 2}}{e^{2 A}}}+\frac{x}{2} \frac{\partial h}{\partial \lambda} \frac{V_{f} \gamma^{2} e^{-2 A} A^{\prime 2}}{\sqrt{1+h \gamma^{2} \frac{A^{\prime 2}}{e^{2 A}}}}\right] \\
& -\frac{1}{h} \frac{\partial \log V_{f}}{\partial T}+\left(V_{g}-x V_{f} \sqrt{1+h \gamma^{2} \frac{A^{\prime 2}}{e^{2 A}}}\right) \frac{\gamma}{6}+\left(4+\beta \frac{\partial \log V_{f}}{\partial \lambda}+\frac{\beta}{2} \frac{\partial \log h}{\partial \lambda}\right) h \gamma^{3} \frac{A^{\prime 4}}{e^{4 A}}(7.6) \\
& +\left[\gamma \frac{\partial \gamma}{\partial T}+\beta \frac{\partial \gamma}{\partial \lambda}+\left(2\left(1-\frac{\beta^{2}}{9 \lambda^{2}}\right)+\beta \frac{\partial \log \left(h V_{f}\right)}{\partial \lambda}\right) \gamma+\left(\frac{1}{2} \frac{\partial \log h}{\partial T}-\frac{\partial \log V_{f}}{\partial T}\right) \gamma^{2}\right] \frac{A^{\prime 2}}{e^{2 A}}=0
\end{aligned}
$$

where we eliminated $A^{\prime \prime}$ by using (7.4). This is a system of two first-order partial differential equations for $\beta, \gamma$, with inputs $V_{f}, V_{g}, h$. The equations are highly non-linear. Setting $x=0$, the system reduces to

$$
\begin{gathered}
12\left(1-\frac{\beta^{2}}{9 \lambda^{2}}\right) \frac{A^{\prime 2}}{e^{2 A}}=V_{g} \\
\left(\gamma \frac{\partial \beta}{\partial T}+\beta \frac{\partial \beta}{\partial \lambda}-\frac{\beta^{2}}{\lambda}\right) \frac{A^{\prime 2}}{e^{2 A}}=-\frac{3}{8} \lambda^{2} \frac{\partial V_{g}}{\partial \lambda}-\frac{V_{g}}{3} \beta .
\end{gathered}
$$

In this case $\frac{\partial \beta}{\partial T}=0$ and (7.7) can be rewritten as

$$
\frac{\partial \beta}{\partial \lambda}=\frac{\beta}{\lambda}-\left(4+\frac{9 \lambda^{2}}{2 \beta} \frac{\partial \log V_{g}}{\partial \lambda}\right)\left(1-\frac{\beta^{2}}{9 \lambda^{2}}\right) .
$$

These equations are similar to those in the probe (quenched) limit.

Note also that although the equations of motion are linear in $x$, the $\beta$-system (7.5), (7.6) is non-linear in $x$, with the understanding that $\frac{A^{\prime 2}}{e^{2 A}}$ is to be eliminated using (7.3).

\footnotetext{
${ }^{12}$ Standard "linear" scalar theories with action given by a kinetic term plus a potential have a unique solution for $\frac{A^{\prime 2}}{e^{2 A}}$.
} 


\subsection{The UV fixed point}

Near the boundary where $\lambda, T \rightarrow 0$, we expect that $\gamma \rightarrow 0$, so that equation (7.3) becomes

$$
12\left(1-\frac{\beta^{2}}{9 \lambda^{2}}\right) \frac{A^{\prime 2}}{e^{2 A}}=V_{g}-x V_{f}(\lambda, 0)+\cdots \quad \rightarrow \quad \frac{A^{\prime 2}}{e^{2 A}}=\frac{V_{g}-x V_{f}(\lambda, 0)}{12\left(1-\frac{\beta^{2}}{9 \lambda^{2}}\right)}+\cdots
$$

According to the discussion of section 3 , the potentials are expected to be regular at $\lambda=0$,

$$
\begin{aligned}
V_{g} & =V_{0}+V_{1} \lambda+V_{2} \lambda^{2}+\mathcal{O}\left(\lambda^{3}\right) \\
x V_{f} & =W_{0}+W_{1} \lambda+W_{2} \lambda^{2}+\mathcal{O}\left(\lambda^{3}\right)+\left(Z_{0}+Z_{1} \lambda+Z_{2} \lambda^{2}\right) T^{2}+\cdots \\
h & =h_{0}+h_{1} \lambda+h_{2} \lambda^{2}+\mathcal{O}\left(\lambda^{3}\right)+\mathcal{O}\left(T^{2}\right) .
\end{aligned}
$$

We also take the following Ansatz for the $\beta$-functions:

$$
\beta=-b_{0} \lambda^{2}+b_{1} \lambda^{3}+\cdots+\mathcal{O}\left(T^{2}\right)+\cdots, \quad \gamma=\left(\gamma_{0}+\gamma_{1} \lambda+\gamma_{2} \lambda^{2}\right) T+\cdots
$$

Inserting these into the equations (7.5), (7.6), we find

$$
\begin{aligned}
& b_{0}=\frac{9}{8} \frac{V_{1}-W_{1}}{V_{0}-W_{0}}, \quad b_{1}=\frac{207}{256} \frac{\left(V_{2}-W_{2}\right)^{2}}{\left(V_{0}-W_{0}\right)^{2}}-\frac{9}{4} \frac{V_{1}-W_{1}}{V_{0}-W_{0}} \\
& \gamma_{0}^{2}+4 \gamma_{0}-\frac{24 Z_{0}}{h_{0} W_{0}\left(V_{0}-W_{0}\right)}=0 \\
& \gamma_{1}=\frac{12 Z_{0}}{\left(\gamma_{0}+2\right) h_{0} W_{0}\left(V_{0}-W_{0}\right)}\left(\frac{Z_{1}}{Z_{0}}-\frac{W_{1}}{W_{0}}-\frac{h_{1}}{h_{0}}-\frac{V_{1}-W_{1}}{V_{0}-W_{0}}\right) .
\end{aligned}
$$

To leading order the solutions around the UV fixed point are

$$
\frac{1}{\lambda}=\frac{1}{\lambda_{0}}+b_{0} A+\cdots, \quad T=T_{0} e^{\gamma_{0} A} .
$$

In order for $T$ to have the proper UV dimension we must have $\gamma_{0}=-1$. In the massless case, $T$ is dominated by the vev, and we have to choose $\gamma_{0}=-3$. These solve eq. (7.14) if

$$
\frac{24 Z_{0}}{h_{0} W_{0}\left(V_{0}-W_{0}\right)}=-3
$$

As we shall point out below, the combination $\gamma(\lambda, T) / T$ is mapped to the anomalous dimension of the quark mass in QCD (2.8). Remarkably, the solution (7.12) is consistent with QCD perturbation theory: $\gamma(\lambda, T) / T$ has a series expansion in $\lambda$. The leading term is fixed according to the UV dimension, and the correction terms are identified with the anomalous dimension.

\subsection{Confining IR asymptotics}

In the IR, a confining asymptotic has the property that $\frac{A^{\prime 2}}{e^{2 A}} \rightarrow \infty$. There are two possibilities for the tachyon: 
1. If $T=0, \gamma=0$ and then $V_{g} \rightarrow V_{g}-x V_{f}(T=0)$. In this case

$$
\frac{A^{\prime 2}}{e^{2 A}}=\frac{V_{g}-x V_{f}(\lambda, 0)}{12\left(1-\frac{\beta^{2}}{9 \lambda^{2}}\right)}+\cdots
$$

2. $T \rightarrow \infty$ in the IR, $V_{f} \rightarrow 0$ exponentially, and we obtain the pure YM case in the IR. Here

$$
\frac{A^{\prime 2}}{e^{2 A}}=\frac{V_{g}}{12\left(1-\frac{\beta^{2}}{9 \lambda^{2}}\right)}+\cdots
$$

If we assume that $V_{f}(\lambda, 0)$ does not grow faster than $V_{g}(\lambda)$ in the IR, the solution in both cases is the same as in pure YM $(x=0),[71-73]$,

$$
\beta \simeq-\frac{3 \lambda}{2}\left[1+\frac{3}{8} \frac{1}{\log \lambda}+\cdots\right]
$$

This non-perturbative $\beta$-function indicates that the scaling dimension of the dual operator $\operatorname{tr}\left(F^{2}\right)$ in the IR is $\Delta=\frac{5}{2}$ read from its linear term. However this does not imply there is a scaling regime in the IR, as the metric is far from AdS.

The tachyon equation becomes

$$
\begin{aligned}
& -\frac{\partial \log V_{f}}{\partial T}+h\left(V_{g}-x V_{f} \sqrt{1+h \gamma^{2} \frac{A^{\prime 2}}{e^{2 A}}}\right) \frac{\gamma}{6}+\left(4+\beta \frac{\partial \log V_{f}}{\partial \lambda}+\frac{\beta}{2} \frac{\partial \log h}{\partial \lambda}\right) \frac{h^{2} V_{g}^{2} \gamma^{3}}{144\left(1-\frac{\beta^{2}}{9 \lambda^{2}}\right)^{2}} \\
& +\left[\gamma \frac{\partial \gamma}{\partial T}+\beta \frac{\partial \gamma}{\partial \lambda}+\left(2\left(1-\frac{\beta^{2}}{9 \lambda^{2}}\right)+\beta \frac{\partial \log \left(h V_{f}\right)}{\partial \lambda}\right) \gamma+\left(\frac{1}{2} \frac{\partial \log h}{\partial T}-\frac{\partial \log V_{f}}{\partial T}\right) \gamma^{2}\right] \\
& \times \frac{h V_{g}}{12\left(1-\frac{\beta^{2}}{9 \lambda^{2}}\right)}=0 .
\end{aligned}
$$

In the IR, for a class of potentials $\gamma \rightarrow-\infty$ and $\sqrt{1+h \gamma^{2} \frac{A^{\prime 2}}{e^{2 A}}} \rightarrow-\sqrt{\frac{h V_{g}}{12\left(1-\frac{\beta^{2}}{9 \lambda^{2}}\right)}} \gamma$. We expect that $V_{f} \sqrt{1+h \gamma^{2} \frac{A^{\prime 2}}{e^{2 A}}} \rightarrow 0$ as $V_{f}$ vanishes exponentially while $\gamma$ increases polynomially with increasing $T$. We also expect $h V_{g}$ to be approximately constant and $\left(1-\frac{\beta^{2}}{9 \lambda^{2}}\right) \rightarrow \frac{3}{4}$. We also expect $\frac{\partial \log h}{\partial T} \rightarrow 0$ and $\beta \frac{\partial \gamma}{\partial \lambda}$ to be subleading.

Therefore the leading terms in equation (7.20) are expected to be

$$
\left(4+\beta \frac{\partial \log V_{f}}{\partial \lambda}+\frac{\beta}{2} \frac{\partial \log h}{\partial \lambda}\right) \frac{h V_{g} \gamma^{3}}{9}+\gamma \frac{\partial \gamma}{\partial T}-\frac{\partial \log V_{f}}{\partial T} \gamma^{2}=0 .
$$

If we define $\delta=\frac{1}{\gamma}, \delta$ satisfies a linear equation

$$
\frac{\partial \delta}{\partial T}+\frac{\partial \log V_{f}}{\partial T} \delta=\frac{h V_{g}}{9}\left(4+\beta \frac{\partial \log \left(V_{f} \sqrt{h}\right)}{\partial \lambda}\right)
$$

with solution

$$
\delta=\frac{C}{V_{f}}+\frac{h V_{g}}{9 V_{f}} \int_{T_{*}}^{T} V_{f}\left(4+\beta \frac{\partial \log \left(V_{f} \sqrt{h}\right)}{\partial \lambda}\right) d T
$$


with $T^{*}$ large enough so that we are in the IR regime. For $\gamma$ we obtain

$$
\gamma=\frac{V_{f}}{C+\frac{h V_{g}}{9} \int_{T_{*}}^{T} V_{f}\left(4+\beta \frac{\partial \log \left(V_{f} \sqrt{h}\right)}{\partial \lambda}\right) d T} .
$$

Note that if $h \sim \lambda^{-\frac{4}{3}}$, then $\beta \frac{\partial \log (\sqrt{h})}{\partial \lambda} \simeq 1$. If we also assume that $V_{f}$ has factorized dependence, $V_{f}=V_{f \lambda}(\lambda) V_{f T}(T)$ we obtain

$$
\gamma=\frac{V_{f}}{C+\frac{h V_{g}}{9}\left(5+\beta \frac{\partial \log \left(V_{f \lambda}\right)}{\partial \lambda}\right) \int_{T_{*}}^{T} V_{f} d T} .
$$

Note that from the whole one-family of solutions above, only one is diverging for large $T$ and this is the only reliable solution.

For a tachyon potential of the form $V_{f T}=e^{-a T^{2}}$ the diverging solution is

$$
\gamma \simeq-\frac{18 a}{h V_{g}\left(5+\beta \frac{\partial \log \left(V_{f \lambda}\right)}{\partial \lambda}\right)} T+\cdots .
$$

For the type of potentials we will be using $h V_{g} / \sqrt{\log \lambda}$ approaches a constant which we denote by $b$, and $V_{f \lambda} \sim \lambda^{2}$ so that

$$
\gamma \simeq-\frac{18 a}{b \sqrt{\log \lambda}\left(2-\frac{9}{8} \frac{1}{\log \lambda}+\cdots\right)} T+\cdots \simeq-\frac{9 a}{b \sqrt{\log \lambda}} T+\cdots
$$

Note that if we assume a more elaborate tachyon potential of the form $V_{f}=$ $V_{f 0}(\lambda) e^{-a(\lambda) T^{2}}$ with $a(\lambda)$ increasing with $\lambda$ in the IR then the asymptotic behavior of $\gamma$ changes and it vanishes in the IR. To capture this behavior from (7.20) we take $\gamma \rightarrow 0$ in the IR and $\sqrt{1+h \gamma^{2} \frac{A^{\prime 2}}{e^{2 A}}} \rightarrow 1$. We also again expect that $h V_{g}$ is approximately constant, $\left(1-\frac{\beta^{2}}{9 \lambda^{2}}\right) \rightarrow \frac{3}{4}$, and $\frac{\partial \log h}{\partial T} \rightarrow 0$. We also expect that the derivative terms $\gamma \frac{\partial \gamma}{\partial T}$ and $\beta \frac{\partial \gamma}{\partial \lambda}$ are subleading.

The two leading terms in (7.20) are then expected to be

$$
-\frac{\partial \log V_{f}}{\partial T}+\frac{h V_{g} \beta}{9} \frac{\partial \log \left(h V_{f}\right)}{\partial \lambda} \gamma=0 .
$$

The solution behaves for large $\mathrm{T}$ as

$$
\gamma \simeq \frac{18 a(\lambda)}{\beta h V_{g} a^{\prime}(\lambda)} \frac{1}{T}+\cdots \simeq-\frac{12}{b \frac{d \log a}{d \log \lambda} \sqrt{\log \lambda}} \frac{1}{T}+\cdots
$$

where $b$ is defined as above.

\subsection{Some simple $\beta$ functions}

We may engineer a $\beta$ function that interpolates between the one-loop perturbative YM $\beta$-function, and the non-perturbative one in (7.19). We could also have a $\gamma$ function interpolating between an operator with dimension $\Delta_{\mathrm{UV}}$ in the UV, and $\Delta_{\mathrm{IR}}$ in the IR:

$$
\beta(\lambda)=-b_{0} \frac{\lambda^{2}}{1+\frac{2 b_{0}}{3} \lambda}, \quad \gamma=\left(\Delta_{\mathrm{UV}}-4\right) T \frac{1+\left(\Delta_{\mathrm{IR}}-4\right) T^{2}}{1+\left(\Delta_{\mathrm{UV}}-4\right) T^{2}} .
$$



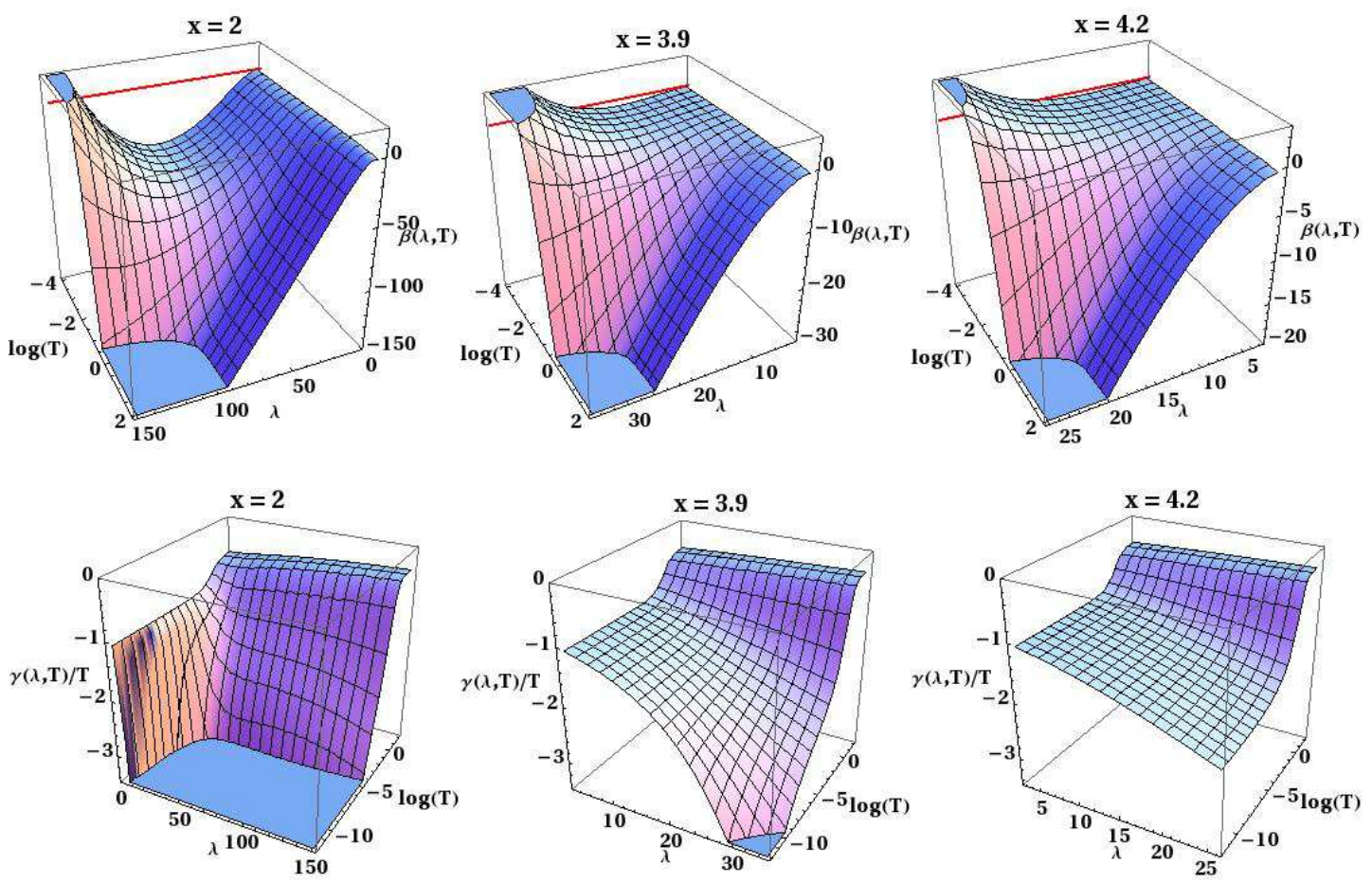

Figure 1. The solutions for the $\beta$ (top row) and $\gamma$ (bottom row) functions for $x=2$ (left), $x=3.9$ (middle), and $x=4.2$ (right). We added the red lines on the top row at $\beta=0$ in order to show the location of the fixed point. See the text for a detailed explanation.

The flow equations can be integrated to

$$
\frac{1}{\lambda}-\frac{2 b_{0}}{3} \log \lambda=b_{0} A, \quad \frac{\log T}{\left(\Delta_{\mathrm{UV}}-4\right)}+\frac{\Delta_{\mathrm{UV}}-\Delta_{\mathrm{IR}}}{2\left(\Delta_{\mathrm{UV}}-4\right)\left(\Delta_{\mathrm{IR}}-4\right)} \log \left(1+\left(\Delta_{\mathrm{IR}}-4\right) T^{2}\right)=A .
$$

Such $\beta$ functions can be converted into potentials $V_{g}, V_{f}$, via the equations (7.5), (7.6).

\subsection{Numerical solutions for the $\beta$ and $\gamma$ functions}

The equations (7.3), (7.5), and (7.6) for the $\beta$ and $\gamma$ functions can be solved numerically for fixed potentials by combining solutions evaluated along the RG flow, as detailed in appendix A. Figure 1 shows the results for various values of $x$. We used the potentials of scenario I from appendix $\mathrm{C}$ and required that the solutions flow to the good IR singularity, as explained in appendix A. Notice that the values $x=3.9$ and 4.2 were chosen to lie slightly above and below the edge of the conformal window, which is at $x_{c} \simeq 3.9959$ for the potentials used in the plots.

The plots of the $\beta$-functions show a smooth transition as $T$ evolves from small $\ll 1$ to large $\gtrsim 1$ values, reflecting the expectation of section 6 . For small tachyon, the $\beta$-function has a fixed point corresponding to the maximum of the effective potential $V_{\text {eff }}$, which moves to lower values of $\lambda$ as $x$ is increased, whereas for large $T$ the $\lambda$-dependence of the $\beta$-function approaches the Yang-Mills form. The $\gamma$ functions show a transition between the small and 
large $T$ regions as well. For large $T$ and $\lambda$, the solutions agree with eq. (7.27). For small $T$, the structure is richer. For $x=2$ the $\gamma$ tends to a constant value as $T \rightarrow 0$ (except for very small $\lambda$ ) so that $\gamma(\lambda, T) / T$, which is plotted in figure 1 , diverges. This behavior is pushed for larger $\lambda$ as we increase $x$ to 3.9 , and has disappeared for $x=4.2$, and the $\gamma$-function is instead linear in $T$ in accordance with eq. (7.12). These changes are related to the tachyon changing sign during the flow. If the tachyon has a zero, we hit the $T=0$ line before reaching the UV singularity. Then $\gamma$ approaches a constant value as $T \rightarrow 0$, and eq. (7.12) does not apply. As will be discussed in detail below, tachyon zeroes are indeed expected for $x<x_{c}$. Notice also that $\gamma / T$ tends to -1 as $\lambda \rightarrow 0$ with fixed $T$ at least for small $T$, reflecting the expected value of $\gamma_{0}$ in eq. (7.12).

\section{The background solutions}

\subsection{Generic properties of the background}

We start by discussing the symmetries and integration constants of the equations of motion of V-QCD, (5.8)-(5.11). We shall assume the exponential Ansatz

$$
V_{f}(\lambda, T)=V_{f 0}(\lambda) \exp \left[-a(\lambda) T^{2}\right]
$$

for the potential of the tachyon DBI action. Then the background EoMs have the following symmetries

$$
\begin{aligned}
& \text { 1. } A \rightarrow A+\log \Lambda, \quad V_{g} \rightarrow \Lambda^{-2} V_{g}, \quad V_{f 0} \rightarrow \Lambda^{-2} V_{f 0} \\
& T \rightarrow \Lambda T, \quad a \rightarrow \Lambda^{-2} a \text {; } \\
& \text { 2. } \quad T \rightarrow \Lambda T, \quad a \rightarrow \Lambda^{-2} a, \quad h \rightarrow \Lambda^{-2} h ; \\
& \text { 3. } \quad r \rightarrow \Lambda\left(r-r_{0}\right), \quad A \rightarrow A-\log \Lambda .
\end{aligned}
$$

The first symmetry can be used to fix the value of the UV AdS radius $\ell$, and is usually associated with the units of energy in the boundary theory. The second one will be used to fix the normalization of $h$ in the UV. The third one is essentially different from the first two, since it does not involve the potentials. It will therefore remain as a true symmetry of the background solutions.

We may choose a set of independent equations of motion which contains one first order and two second order differential equations. Therefore, their general solution includes five integration constants. These can be identified as the coefficients of the UV expansions of the fields as follows (assuming that the solution has the standard UV singularity with $\lambda \rightarrow 0$, see appendix D). The tachyon UV expansion has the usual free constants related to the normalizable and non-normalizable solutions, identified as the quark mass $m$ and the vacuum expectation value $\sigma$ of the $\bar{q} q$ operator, respectively.

In close analogy, the solution for $\lambda$ involves two constants, identified as the UV scale $\Lambda=\Lambda_{\mathrm{UV}}$ of the expansions, and another constant $\hat{A}$ which we will define in section 9 , related to the gluon condensate and the free energy of the system. The fifth integration constant is simply the location of the UV singularity which can take to be $r=0$ by the translation symmetry of eq. (8.4). We shall require that the system has a repulsive, "good" kind of IR singularity, which fixes the values of the condensates $\sigma$ and $\hat{A}$ in terms of $m$ and $\Lambda_{\mathrm{UV}}$. 
In addition, we still have the scaling symmetry of eq. (8.4), which can be used to vary the units of all constants, and reflects the corresponding scale transformation of the dual field theory. Therefore, the single parameter which characterizes all nontrivially linked physical backgrounds (for fixed potentials and, in particular fixed $x=N_{f} / N_{c}$ ) is the ratio of the "source" coefficients $m / \Lambda_{\mathrm{UV}} \cdot{ }^{13}$

For the choices of the functions $h, V_{g}$, and $V_{f}$ of interest to us, the tachyon typically decouples from the other fields asymptotically in the UV and in the IR. The UV and IR asymptotics are discussed in detail in appendices $\mathrm{D}$ and $\mathrm{E}$, and we shall repeat only the main features of the physically interesting possibilities here. The physically relevant UV asymptotics are restricted. As pointed out in [71-73], the fields $A$ and $\lambda$ can be expanded in $-1 / \log r$ at the UV boundary $r=0$ in the probe limit.

Similar expansions work also at finite $x=N_{f} / N_{c}$. The tachyon is required to vanish linearly $T(r) \simeq m r$ (or faster if $m=0$ ) in $r$ in the UV. Taking $\epsilon=-1 / \log r \rightarrow 0$, the tachyon $T \simeq m \exp (-1 / \epsilon)$ vanishes exponentially while $A$ and $\lambda$ have power-like behavior in $\epsilon$. Since, in addition, the functions $h$ and $V_{f}$ must be regular in the UV (see section 8.2 below), the tachyon can be set to zero in the leading order action. We find that $A$ and $\lambda$ satisfy their probe limit equations of motion, but with the dilaton potential $V_{g}$ replaced by $V_{\text {eff }}(\lambda)=V_{g}(\lambda)-x V_{f}(\lambda, T=0)$, which also verifies that the UV expansions of $\lambda$ and $A$ have the same form as in the probe limit.

We shall only discuss cases where the tachyon indeed decouples asymptotically in the IR. This is the case, if we take a tachyon potential having an exponential $T$ dependence, $V_{f}(\lambda, T) \propto \exp \left(-a T^{2}\right)$, and the tachyon has a power-law (or faster) divergence as $r \rightarrow \infty$. This is indeed what is suggested by tachyon condensation in string theory. Therefore, the flavor part of the action is exponentially suppressed in the IR. Consequently, the tachyon decouples asymptotically from $A$ and $\lambda$, and their asymptotic expansions in the IR have exactly the same form as in the probe limit, and are determined by the potential $V_{g}(\lambda)$.

In summary, even though all fields couple nontrivially for general values of the coordinate, the probe limit description will be valid in the UV and IR. In particular, this guarantees that the interpretation of the integration constants is the same for finite $x$ as in the probe limit. The decoupling of the tachyon in the IR leads to the system having similar "good" IR singularities as in the probe case.

An important difference with respect to the probe limit discussion is that the potentials which characterize the backgrounds in the UV and IR regions will be, in general, qualitatively different. As discussed above in section 6, the potential $V_{\text {eff }}(\lambda)=V_{g}(\lambda)-x V_{f}(\lambda, T=0)$ which controls the UV behavior will be chosen such that it admits a fixed point (extremum of the potential) at least for large values of $x$ as required by the Banks-Zaks analysis.

The fixed point of $V_{\text {eff }}$ will play an important role in the dynamics in the intermediate region between UV and IR. In particular, for identically vanishing tachyon, the background simply flows from the UV fixed point at $\lambda=0$ to the IR fixed point at finite $\lambda$. Adding, a

\footnotetext{
${ }^{13}$ Due to practical reasons we shall often use a parameter $T_{0}$ linked to the tachyon behavior in the IR instead of the quark mass to characterize different backgrounds.
} 
tiny quark mass (or chiral condensate), the solution in the UV region will not be changed drastically. However, no matter how small the quark mass is, the tachyon will eventually become large and start coupling to $\lambda$ and $A$, which will drive the system away from the IR fixed point.

We can make two observations. First, the special case of zero tachyon will be essentially different from all other solutions. This solution has vanishing quark mass and chiral condensate, and is therefore identified as the solution corresponding to conserved chiral symmetry. We will discuss this special case separately below.

Second, there is a possibility to have solutions which come very close to the IR fixed point, but are eventually driven away by the increasing tachyon. Such solutions will be identified with quasiconformal or "walking" dynamics of the dual field theory, where the coupling constant remains approximately fixed over a large range of energies. We shall see below how the phase structure of QCD in the Veneziano limit, which includes a quasiconformal region, arises in our class of models.

\subsection{Matching UV behavior with the QCD $\beta$-functions}

We shall now discuss the most important links of the potential functions to the physics of the dual field theory. We start by an analysis of the UV region, where the behavior of the system can be mapped to the QCD $\beta$-functions [71-73] as already discussed in section 7 . In particular, in the probe limit $\left(x \rightarrow 0\right.$ limit), the UV behavior is controlled by $V_{g}(\lambda)$, that has the expansion

$$
V_{g}(\lambda)=V_{0}+V_{1} \lambda+V_{2} \lambda^{2}+\cdots
$$

Here $V_{0}>0$ can be freely chosen and it fixes the AdS scale for $x=0$ as $V_{0}=12 / \ell_{0}^{2}$. The other coefficients can be mapped to the Yang-Mills $\beta$-function. At one-loop order we have [71-73]

$$
V_{g}(\lambda)=\frac{12}{\ell_{0}^{2}}\left[1+\frac{8}{9} b_{0}^{\mathrm{YM}} \lambda+\cdots\right]
$$

where $b_{0}{ }^{\mathrm{YM}}$ is the one-loop coefficient of the $\beta$-function, from which $V_{1}$ can be solved.

Moreover, as discussed above, at finite $x$ the UV behavior is similar to the probe limit, but the role of $V_{g}$ is taken by the potential $V_{\text {eff }}(\lambda)=V_{g}(\lambda)-x V_{f}(\lambda, T=0)$. Therefore, we take

$$
V_{f}(\lambda, T=0)=W_{0}+W_{1} \lambda+W_{2} \lambda^{2}+\cdots
$$

and the relation to the QCD $\beta$-function reads

$$
V_{\text {eff }}(\lambda)=V_{g}(\lambda)-x V_{f}(\lambda, 0)=\frac{12}{\ell^{2}}\left[1+\frac{8}{9} b_{0} \lambda+\cdots\right]
$$

where $b_{0}$ is the leading coefficient of the $\beta$-function in the Veneziano limit.

Similarly to $V_{0}$, the coefficient $W_{0}$ can be freely chosen, and the other coefficients can be solved from eq. (8.8). However, there are constraints: the AdS scale must remain 
positive for all $0<x<11 / 2$, and $W_{0}$ should also be positive (see appendix C). These boil down to

$$
0<W_{0}<\frac{2}{11} V_{0}
$$

If we allow $W_{0}$ to depend on $x$, the upper limit is modified to $V_{0} / x$.

In addition, as pointed out in section 7 , we can map the UV behavior of the tachyon action to the (anomalous) dimensions of the quark mass and the chiral condensate of the dual field theory. For definiteness, we parametrize $V_{f}(\lambda, T)=V_{f 0}(\lambda) \exp \left(-a(\lambda) T^{2}\right)$ and assume that $h$ depends only on $\lambda$. Then the dimension of the quark mass constrains the UV behavior of $h(\lambda)$ and $a(\lambda)$. A detailed analysis is done in appendix D.1.1.

Remarkably, assuming that the potential functions have analytic expansions at $\lambda=0$ is consistent with QCD perturbation theory. Indeed, requiring the dimension of the quark mass to approach one in the deep UV fixes the leading terms as

$$
\frac{h(\lambda)}{a(\lambda)}=\frac{2 \ell^{2}}{3}\left(1+h_{1} \lambda+\cdots\right)
$$

and the next-to-leading coefficient $h_{1}$ can be matched with the one-loop anomalous dimension of the quark mass $\gamma_{m}(\lambda)$.

By using eq. (D.10) from appendix, we obtain

$$
-\gamma_{0}=\frac{9}{8}\left[\frac{4}{3} \frac{8}{9} b_{0}+\frac{4}{3} h_{1}\right]
$$

where $\gamma_{0}$ is the leading coefficient of the anomalous dimension, $-d \log m / d \log \mu=\gamma_{m}(\lambda)=$ $\gamma_{0} \lambda+\cdots$. Notice that, for example, the non-normalizable term in the tachyon solution (D.9) reads after this identification

$$
\frac{1}{\ell} T(r)=m r(-\log (r \Lambda))^{-\gamma_{0} / b_{0}}\left[1+\mathcal{O}\left(\frac{1}{\log (r \Lambda)}\right)\right]
$$

where the logarithmic correction is consistent with the one-loop solution for the running quark mass in QCD.

\subsection{Condensate dimension and the edge of the conformal window}

The most important new feature of the system discussed in this article is the description of the phase diagram of QCD as a function of $x=N_{f} / N_{c}$ in the Veneziano limit. We shall now indicate which potentials lead to the desired structure. This constraint to a large extent independent of the one discussed above that was set by the UV expansions.

The phase structure is linked to the dimension of the chiral condensate at the IR fixed point which is found at the maximum of $V_{\mathrm{eff}}=V_{g}-x V_{f 0}$ in the conformal window (in the limit of small tachyon background).

We denote the value of the coupling at the fixed point by $\lambda_{*}$ so that

$$
V_{\mathrm{eff}}^{\prime}(\lambda)=V_{g}^{\prime}\left(\lambda_{*}\right)-x V_{f 0}^{\prime}\left(\lambda_{*}\right)=0 \text {. }
$$


From the action (5.5) we can calculate the IR AdS scale

$$
\frac{12}{\ell_{\mathrm{IR}}^{2}}=V_{\mathrm{eff}}\left(\lambda_{*}\right)
$$

To calculate the dimension we need the mass of $T$ in the IR fixed point, which can be extracted from the action (5.3) and reads

$$
m_{\mathrm{IR}}^{2}=-\frac{2 a\left(\lambda_{*}\right)}{h\left(\lambda_{*}\right)}
$$

where we again parametrized $V_{f}(\lambda, T)=V_{f 0}(\lambda) \exp \left(-a(\lambda) T^{2}\right)$. From this we obtain

$$
\Delta_{\mathrm{IR}}\left(4-\Delta_{\mathrm{IR}}\right)=-m_{\mathrm{IR}}^{2} \ell_{\mathrm{IR}}^{2}=\frac{24 a\left(\lambda_{*}\right)}{h\left(\lambda_{*}\right) V_{\mathrm{eff}}\left(\lambda_{*}\right)} .
$$

The desired phase diagram is only obtained if the expression $\Delta_{\mathrm{IR}}\left(4-\Delta_{\mathrm{IR}}\right)=-m_{\mathrm{IR}}^{2} \ell_{\mathrm{IR}}^{2}$ has a certain dependence on $x$. It must start at a value smaller than 4 at the end of the BZ region (which is guaranteed as the standard UV boundary conditions fix it to 3 there), grow as $x$ decreases, and become 4 at some value of $x$ so that the BF bound [115] is saturated [7]. Indeed, the solutions near the edge of the conformal window stabilize such that the critical $x_{c}$, defining the location of the conformal phase transition for massless quarks, is determined by

$$
\left.\Delta_{\mathrm{IR}}\left(4-\Delta_{\mathrm{IR}}\right)\right|_{x=x_{c}}=4 .
$$

We shall discuss why this is the case in detail later on. Note that in addition to the explicit dependence on $x$ in the factor $x V_{f 0}$, eq. (8.16) depends on $x$ through $\lambda_{*}$ due to the definition of eq. (8.13).

We stress that the saturation of the BF bound means that for theories near the critical $x_{c}, \Delta_{\mathrm{IR}} \rightarrow 2$, or in other words, the anomalous dimension of the quark mass at the fixed point $\gamma_{*} \rightarrow 1$. That is, our model reproduces the standard assumption for the energy dependence of the chiral condensate near the edge of the conformal window. Recall that this is extremely important for realizations of walking technicolor.

If the potentials are matched with the UV physics of QCD, the expression (8.16) can be further simplified. Fixing the tachyon mass in the deep UV we obtain $a(0) / h(0)=3 /\left(2 \ell^{2}\right)$. In the limit $T \rightarrow 0$, the $\beta$-function $\beta(\lambda)=d \lambda / d A$ satisfies a first order differential equation which depends on $V_{\text {eff }}(\lambda)$, as can be immediately concluded by comparing our action to the probe limit one [71-73] for $T=0$. In terms of the phase function $X$, defined by

$$
\frac{d \lambda}{d A}=\beta(\lambda)=3 \lambda X(\lambda)
$$

we find $[71-73]$

$$
\lambda \frac{d X(\lambda)}{d \lambda}=-\left(8 X(\lambda)+3 \lambda \frac{d \log V_{\mathrm{eff}}(\lambda)}{d \lambda}\right) \frac{1-X^{2}(\lambda)}{6 X(\lambda)} .
$$

Solving this, we can parametrize the potential in terms of the $\beta$-function:

$$
V_{\text {eff }}(\lambda)=V_{g}(\lambda)-x V_{f 0}(\lambda)=\frac{12}{\ell^{2}}\left(1-\frac{\beta(\lambda)^{2}}{9 \lambda^{2}}\right) \exp \left[-\frac{8}{9} \int_{0}^{\lambda} \frac{d \hat{\lambda} \beta(\hat{\lambda})}{\hat{\lambda}^{2}}\right] .
$$


The IR dimensions now satisfy

$$
\begin{aligned}
\Delta_{\mathrm{IR}}\left(4-\Delta_{\mathrm{IR}}\right) & =24 \frac{a\left(\lambda_{*}\right)}{h\left(\lambda_{*}\right)} \frac{\ell^{2}}{12}\left(1-\frac{\beta\left(\lambda_{*}\right)^{2}}{9 \lambda_{*}^{2}}\right)^{-1} \exp \left[\frac{8}{9} \int_{0}^{\lambda_{*}} \frac{d \lambda \beta(\lambda)}{\lambda^{2}}\right] \\
& =3 \frac{h(0)}{h\left(\lambda_{*}\right)} \frac{a\left(\lambda_{*}\right)}{a(0)} \exp \left[\frac{8}{9} \int_{0}^{\lambda_{*}} \frac{d \lambda \beta(\lambda)}{\lambda^{2}}\right] .
\end{aligned}
$$

where in the second line we used the fact that $\beta\left(\lambda_{*}\right)=0$. If we do the matching with perturbative QCD results in the UV, the $\beta$-function can be identified as the QCD $\beta$ function in the Veneziano limit. Then the exponential factor is small in the sense that it is roughly proportional to $b_{0} \lambda_{*}$ where both the one-loop coefficient of the $\beta$-function $b_{0}$ and the coupling at the fixed point $\lambda_{*}$ vanish in the BZ limit $x \rightarrow 11 / 2$. Therefore we expect that $\Delta_{\mathrm{IR}}\left(4-\Delta_{\mathrm{IR}}\right)$ depends on $x$ mostly through the functions $h$ and $a$. In the two-loop approximation of the $\beta$-function we obtain

$$
\frac{8}{9} \int_{0}^{\lambda_{*}} \frac{d \lambda \beta(\lambda)}{\lambda^{2}}=-\frac{4}{9} b_{0} \lambda_{*}
$$

In figure 2 we plot the dependence of $\Delta_{\mathrm{IR}}\left(4-\Delta_{\mathrm{IR}}\right)$ on $x$ for the two scenarios of potential choices of appendix C. The solid lines give the results for the value $W_{0}=12 / 11$ used in the appendix, and the dashed lines show the sensitivity of the result for the choice of $W_{0}$ as this parameter is varied over its allowed range. In all cases, $\Delta_{\mathrm{IR}}\left(4-\Delta_{\mathrm{IR}}\right)$ intersects the value of 4 , shown as the horizontal dotted line in the plot. This suggests that the class of potentials that has the desired phase structure quite in general includes those ones that are matched with the UV behavior of QCD. Moreover, the critical value of $x$ is found within a quite narrow band

$$
3.7 \lesssim x_{c} \lesssim 4.2
$$

and the largest source of uncertainty is the choice of $W_{0} \cdot{ }^{14}$ We stress that there is no strict bound on the allowed values of $x_{c}$, but the numbers in eq. (8.23) rather give the expected magnitude for the variation of $x_{c}$ within natural potential choices.

It is also instructive to show the dependence of the anomalous dimension of the quark mass at the IR fixed point on $x$ (see figure 3 ). Within our model the anomalous dimension is defined by $\gamma_{*}=\Delta_{\mathrm{IR}}-1$ where $\Delta_{\mathrm{IR}}$ is the smaller of the two roots. The result for the potentials of scenario I of appendix $\mathrm{C}$ is shown as the solid blue line in figure 3 , and as in figure 2, the dashed lines show the maximal variation as the parameter $W_{0}$ is varied over its allowed range.

Our result is very similar to the prediction obtained by calculating the anomalous dimension from Dyson-Schwinger equations in the rainbow approximation [116, 117], and evaluating the result at the zero of the perturbative two-loop $\beta$-function (2.3) (dotted red curve of figure 3 ). As in our model, the conformal window ends at the point where $\gamma_{*}$ reaches one and becomes complex in this approach. The deviation from the simple perturbative estimate (dot-dashed magenta curve), which was obtained by using the two-loop anomalous

\footnotetext{
${ }^{14}$ We have checked this also for some additional potentials that are not discussed in this article.
} 


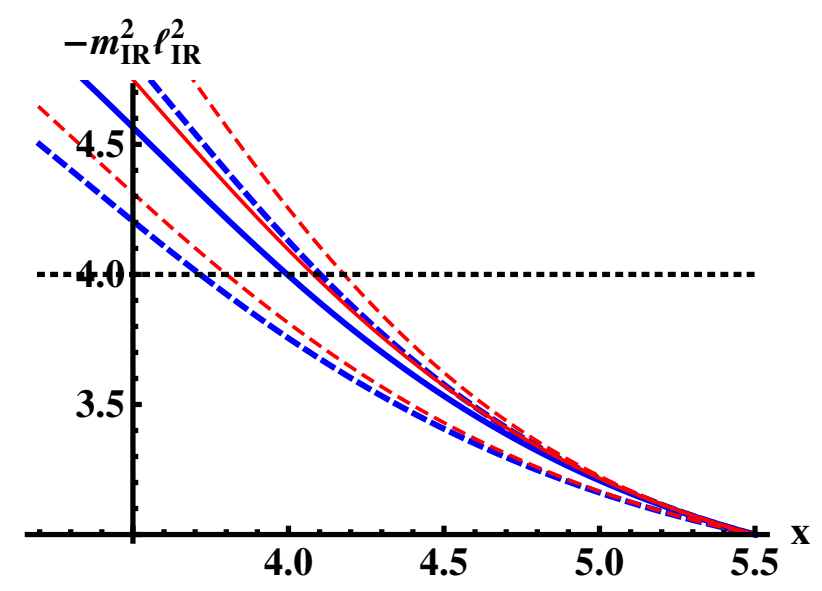

Figure 2. The squared tachyon mass at the IR fixed point $\Delta_{\mathrm{IR}}\left(4-\Delta_{\mathrm{IR}}\right)=-m_{\mathrm{IR}}^{2} \ell_{\mathrm{IR}}^{2}$ as a function of $x$ for the two scenarios described in appendix C. Thick blue, and thin red curves are the results for the scenarios I and II of appendix C, respectively. The dashed lines show the maximal changes as $W_{0}$ is varied from 0 (upper curves) to 24/11 (lower curves).

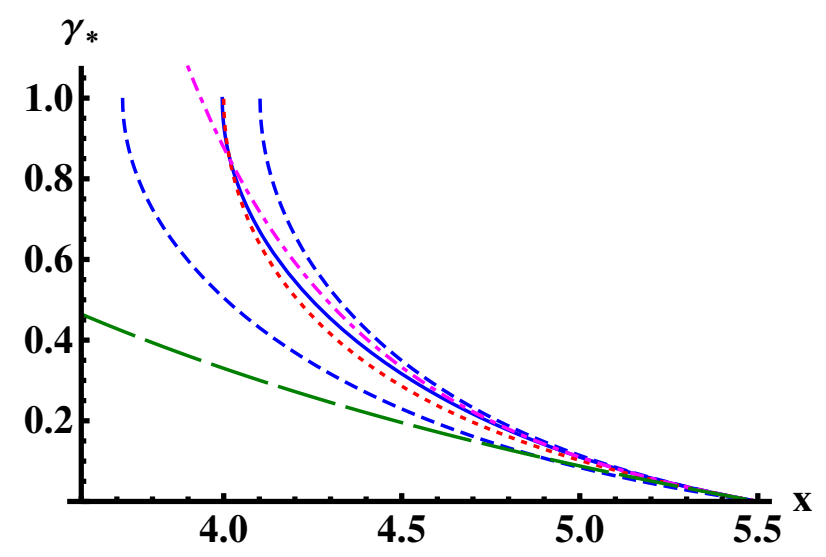

Figure 3. The anomalous dimension of the quark mass at the IR fixed point as a function of $x$ within the conformal window in various approaches. The solid blue curve is our result for the scenario I of appendix C. The dashed blue lines show the maximal change as $W_{0}$ is varied from 0 (upper curve) to 24/11 (lower curve). The dotted red curve is the result from a Dyson-Schwinger analysis, the dot-dashed magenta curve is the prediction of two-loop perturbative QCD, and the long-dashed green curve is based on an all-orders $\beta$-function.

dimension (2.8) instead, is also small. However, an all-orders $\beta$-function [118] (long-dashed green curve) predicts much smaller values of $\gamma_{*}$ for low values of $x$.

In figure 4 (left) we plot the ratio $\frac{\ell_{U V}}{\ell_{\mathrm{IR}}}$ comparing the AdS scales of the IR and UV CFTs. This ratio by unitarity is always larger than one, and reflects the loss of degrees of freedom during the flow. The right hand plot shows the ratio $\frac{1}{\left(1+\gamma_{*}\right)}$ in the conformal window, which controls the dependence of the ratio of the UV and IR scales on the quark mass (see also section 8.6 below). 

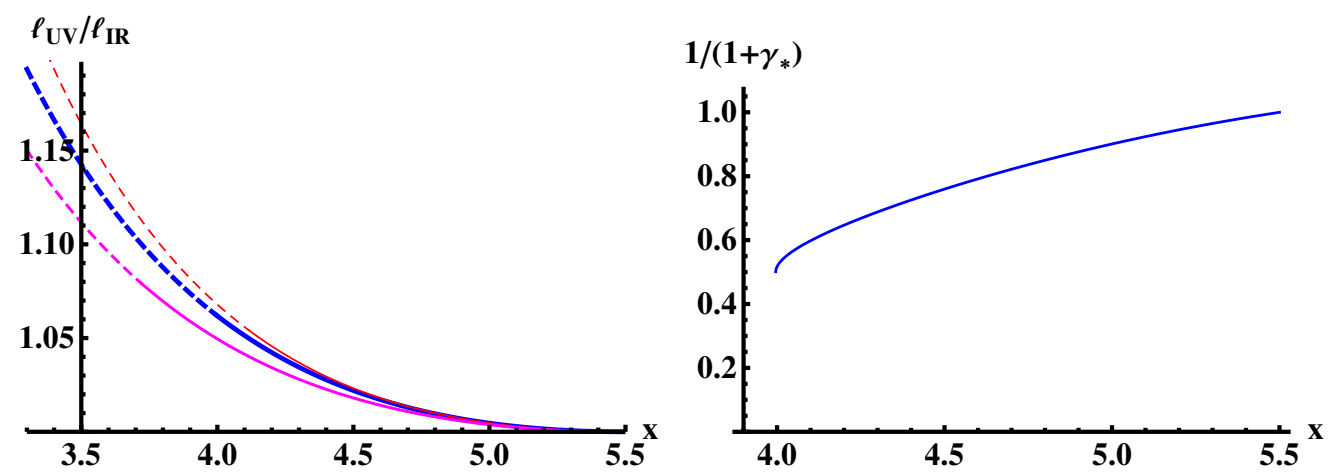

Figure 4. Left: The ratio $\frac{\ell_{\mathrm{UV}}}{\ell_{\mathrm{IR}}}$ comparing the AdS scales of the IR and UV CFTs. This ratio reflects the loss of degrees of freedom during the flow. The blue line was drawn with the potential I, and $W_{0}=\frac{11}{12}$. The other lines correspond to varying the parameter $W_{0}$ in the potential between its extreme values $W_{0}=0$ (red) and $W_{0}=\frac{24}{11}$ (magenta). Right: The ratio $\frac{1}{\left(1+\gamma_{*}\right)}$ as a function of $x$ in the conformal window. It controls the ratio of the UV and IR characteristic scales as $\frac{\Lambda_{\mathrm{IR}}}{\Lambda_{\mathrm{UV}}} \sim\left(\frac{m}{\Lambda_{\mathrm{UV}}}\right)^{\frac{1}{\left(1+\gamma_{*}\right)}}$.

\subsection{Constructing the background solutions}

We will select concrete potentials for evaluating the background numerically. These potentials must satisfy the constraints of the previous two subsections in order to produce the desired phase structure of QCD in the Veneziano limit as well as the perturbative UV physics. In addition, the system needs to have an acceptable IR singularity where the tachyon diverges, which adds extra requirements to the large $\lambda$ behavior of the potentials. However, as we shall demonstrate briefly, different choices for the IR behavior do not change the qualitative features of the background.

The construction of explicit potentials is detailed in appendix C, where also the IR behavior is fixed by using the generic analysis of appendix E. For clarity, we repeat the final result here. The potentials of the scenario I in appendix $\mathrm{C}$ read

$$
\begin{aligned}
V_{g}(\lambda) & =12+\frac{44}{9 \pi^{2}} \lambda+\frac{4619}{3888 \pi^{4}} \frac{\lambda^{2}}{\left(1+\lambda /\left(8 \pi^{2}\right)\right)^{2 / 3}} \sqrt{1+\log \left(1+\lambda /\left(8 \pi^{2}\right)\right)} \\
V_{f}(\lambda, T) & =V_{f 0}(\lambda) e^{-a(\lambda) T^{2}} \\
V_{f 0}(\lambda) & =\frac{12}{11}+\frac{4(33-2 x)}{99 \pi^{2}} \lambda+\frac{23473-2726 x+92 x^{2}}{42768 \pi^{4}} \lambda^{2} \\
a(\lambda) & =\frac{3}{22}(11-x) \\
h(\lambda) & =\frac{1}{\left(1+\frac{115-16 x}{288 \pi^{2}} \lambda\right)^{4 / 3}} .
\end{aligned}
$$

We shall use this choice when calculating the background numerically, unless stated otherwise.

Recall that only three of the equations of motion (5.8)-(5.11) are independent. We choose a set of three second-order equations for the numerical calculations, and treat the 
remaining first order equation as an extra constraint. As usual, we choose boundary conditions that satisfy the constraint, which is then automatically satisfied for all $r{ }^{15}$

When solving the equations numerically, it turns out that shooting from the IR is numerically stable. ${ }^{16}$ We shall first discuss backgrounds where the tachyon has a nontrivial profile. We fix the boundary conditions in the deep IR by using the asymptotic IR expansions at the "good" IR singularity at $r=\infty$ (see appendix E.2.2). For the functions given above, the asymptotics becomes

$$
\begin{aligned}
A(r) & =-\frac{r^{2}}{R^{2}}+\frac{1}{2} \log \frac{r}{R}-\log R+\frac{13}{8}+\log \left[\frac{276^{1 / 4}}{\sqrt{4619}}\right]-\frac{173 R^{2}}{3456 r^{2}}+\cdots \\
\log \lambda(r) & =\frac{3}{2} \frac{r^{2}}{R^{2}}-\frac{39}{16}+\log 8 \pi^{2}-\frac{151 R^{2}}{2304 r^{2}}+\cdots \\
T(r) & \sim T_{0} \exp \left[\frac{813^{5 / 6}(115-16 x)^{4 / 3}(11-x)}{8129442^{1 / 6}} \frac{r}{R}\right] .
\end{aligned}
$$

Here we already used the translation symmetry of eqs. (8.4) to set $r_{0}$ in the formulas (E.18) and (E.20) to zero, ${ }^{17}$ and we can further use the scaling symmetry to fix $R=1$. After this, the solution (for fixed $x$ ) depends nontrivially on only one free parameter $T_{0}$ in the IR, as is characteristic for a good IR singularity.

To continue, we choose an IR cutoff $R_{\mathrm{IR}}$ such that the tachyon is large, and therefore decoupled from the fields $A$ and $\lambda$. With the above choices of potentials, a sufficient value turns out to be $T\left(R_{\mathrm{IR}}\right)=70$ : with this choice, the tachyon has decoupled to a good precision, and $R_{\mathrm{IR}} / R$ is large enough for the asymptotic expansions to work. We use the asymptotic expansions to fix the values of $A, \lambda, T, A^{\prime}$, and $T^{\prime}$ at $r=R_{\mathrm{IR}}$, and solve $\lambda^{\prime}$ from the constraint (the first order EoM). The solution is then obtained by numerically solving the set of second order equations of motion toward the UV, until some of the UV asymptotics described in appendix D are reached.

The obtained UV behavior is depicted in figure 5 (left) as a function of the only remaining free parameters, $T_{0}$ and $x$. For comparison, we also present the same plot for the scenario II of appendix $\mathrm{C}$ on the right hand side, where $T_{0}$ is replaced by the parameter $r_{1}$ of eq. (C.21). For clarity, we shall only refer to the variable $T_{0}$ in the discussion below. Because of the invariance of the Lagrangian under $T \rightarrow-T$, scanning over positive $T_{0}$ is enough to catalog all possible solutions. We shall explain the notation and the results here,

\footnotetext{
${ }^{15}$ In all numerical calculations, we shall do the coordinate transformation from $r$ to $A$ discussed in appendix B, because after this, the UV structure of the solutions is reproduced more accurately. However, as this transformation is straightforward, we shall continue to discuss the solution in the " $r$-space".

${ }^{16}$ There is a potential instability related to the constraint, as it is exactly satisfied only near the IR cutoff due to numerical effects. For general $r$ there is an error which may grow exponentially as we solve the system towards the UV. However, we have the freedom of modifying the system of second order equations, which is used to calculate the solution, by adding multiples of the constraint to some of the equations. In this way the error can be made to decrease exponentially instead of growing towards the UV, so that the instability is removed.

${ }^{17}$ Notice that with this choice of fixing the translation invariance, the UV boundary of the final solution will not be at $r=0$. After obtaining the solution, we can relax this condition and use translation invariance again to move the UV singularity at $r=0$, if desired.
} 

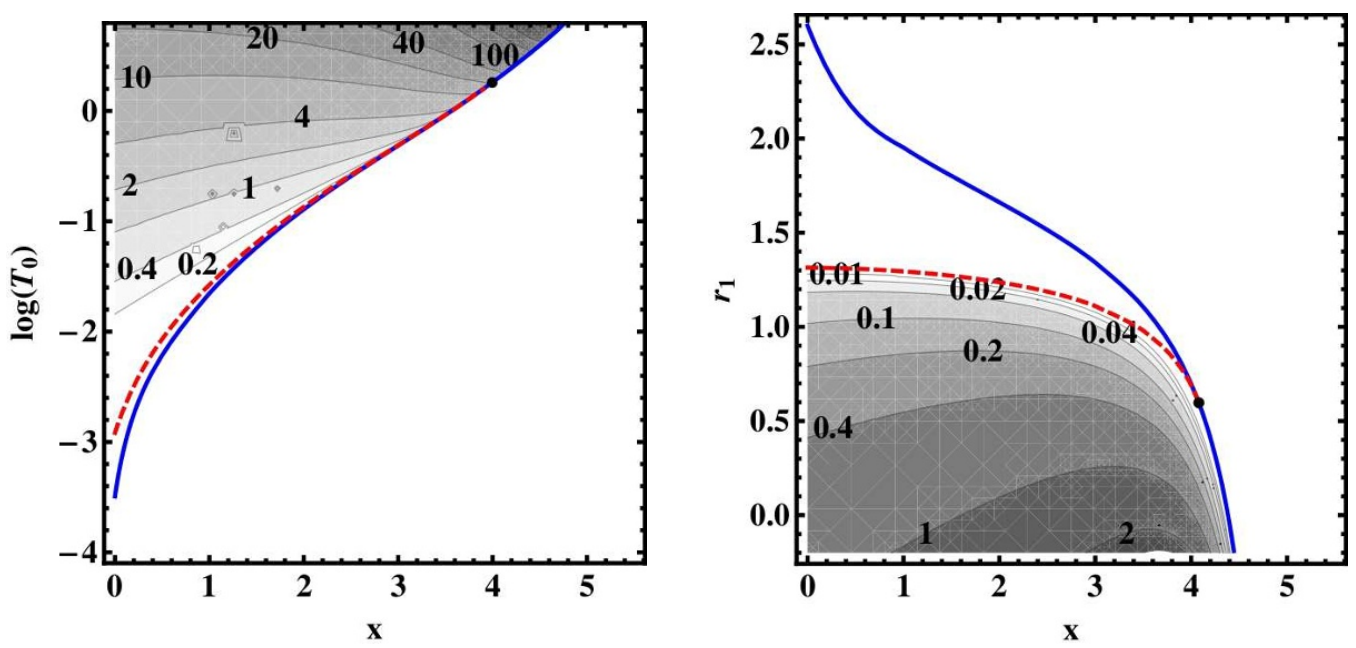

Figure 5. The UV behavior of the background solutions with good IR singularity for the scenario I (left) and scenario II (right) defined in appendix C. The thick blue curve represents a change in the UV behavior, the red dashed curve has zero quark mass, and the contours give the quark mass. The black dot where the zero mass curve terminates lies at the critical value $x=x_{c}$. For scenario I (II) we have $x_{c} \simeq 3.9959$ ( $\left.x_{c} \simeq 4.0797\right)$. See the text for detailed explanation.

and discuss at qualitative level how the structure arises, whereas the details are discussed in appendix F. We shall also show concrete examples of the backgrounds as well as their $\beta$ - and $\gamma$-functions along the holographic RG flow below.

The solid blue line represents a change in the UV asymptotics. The standard UV asymptotics of appendix D.1.1 is obtained left of the blue line, whereas right of the blue line the solution "bounces back" at finite $\lambda$, as discussed in appendix D.1.2. In the bounceback region the $\beta$-function evaluated along the RG flow becomes zero at finite $\lambda$, after which $\lambda$ starts growing toward the UV, and the standard UV boundary is not reached. To the left of the blue solid curve, the "standard" tachyon UV expansion of appendix D.1.1 defines the quark mass and the chiral condensate, which are not defined right of, or on, the blue line. The red dashed line has $m=0$. This line exists only for small $x$ and terminates at a critical value $x_{c} \simeq 3.9959$ (in scenario I), which matches with the definition of eq. (8.17).

We stress that the solution with zero quark mass does not exist for $x \geq x_{c}$. Right of the red dashed line, the quark mass takes positive values and is monotonic in $T_{0}$. The contours give the quark mass, obtained by fitting the deep UV behavior of the tachyon solution to the expansion of appendix D. ${ }^{18}$ The backgrounds in the contoured region will be identified as the physical ones, having lowest free energy.

The value of the mass goes to zero when the solid blue (for $x>x_{c}$ ) or the red dashed (for $x<x_{c}$ ) curves are approached from within the contoured region as shown in figure 6 , which is not evident from figure 5 due to limited resolution. Between the blue solid and red dashed curves, the quark mass is small, but depends on $x$ and $T_{0}$ in a complicated manner, as we shall discuss below.

\footnotetext{
${ }^{18}$ The mass here is given in IR units, i.e., we actually plot $m R=m / \Lambda_{\mathrm{IR}}$, since we fixed the scale $R$ of the IR expansions to unity in the numerics.
} 

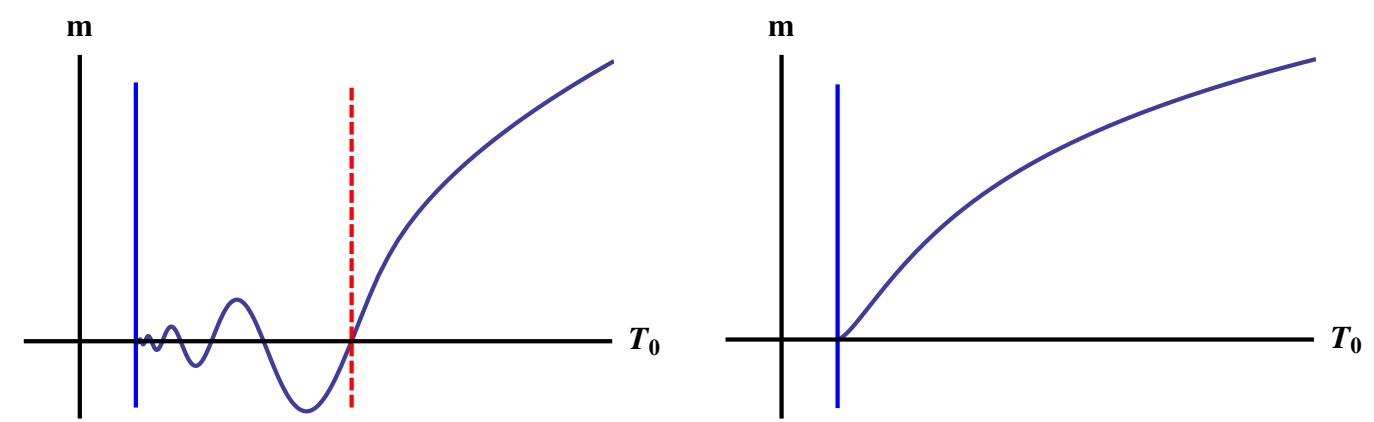

Figure 6. Left figure: Plot of the UV Mass parameter $m$, as a function of the IR $T_{0}$ scale, for $x<x_{c}$. Right figure: Similar plot for $x \geq x_{c}$. The vertical solid blue and dashed red lines show where corresponding lines are intersected in figure 5 . While these plots are for model functions, similar plots on how the bare quark mass $m$ depends on $T_{0}$, for different $x$ for the potentials of scenario I, can be found in figure 17 in appendix F.

The right hand plot in figure 5, which was obtained after modifying the potentials in the IR, shows similar qualitative features as the left hand plot. This is the case because the structure seen in figure 5 arises from the behavior of the solutions in the UV region and close to it, which is analytically tractable and almost independent of the change of the potentials, if, e.g., we keep the quark mass fixed (see the discussion in appendix F). However, the mapping to the IR asymptotics (in particular to $T_{0}$ or $r_{1}$ ) is completely different in the two scenarios, which causes the differences between the plots.

Let us then discuss how the structure of figure 5 arises from the background solutions (see appendix $\mathrm{F}$ for a detailed analysis). The main point is that the closer we are to the thick blue curve (when approaching the curve from the right), the closer the background is to reaching the IR fixed point, when the tachyon finally grows large and drives the system away from it. Therefore the backgrounds near the blue curve will be quasiconformal, or "walking", so that the coupling $\lambda$ is approximately constant over a large range of energies. The mass dependence can be then understood be studying the tachyon EoM and in particular the tachyon mass at the IR fixed point. Notice that as the red dashed curve of solutions with zero quark mass ends on the blue curve as $x \rightarrow x_{c}$, quasiconformal behavior is expected in this region.

To understand the behavior near the blue and red curves it is important to plot the UV parameter, $m$, versus the IR parameter $T_{0}$. We show this in figure 6 for $x<x_{c}$ (left) and $x \geq x_{c}$ (right). ${ }^{19}$ For $x \geq x_{c}$, there is a unique saddle point (regular classical solution) for each value of the quark mass.

The situation for $x<x_{c}$ is more complex and reflects the fact that the chiral condensate operator violates the BF bound in the (potential) IR fixed point. ${ }^{20}$ We see from the left of figure 6 that for each $m>0$, there is a finite number of regular classical solutions that we

\footnotetext{
${ }^{19}$ As $m$ and $-m$ are related by a chiral rotation by $\pi$, we expect that we can take $m \geq 0$. The chiral rotation is reflected in the background solutions in the symmetry $T \rightarrow-T$. Consequently, we can turn the negative mass solutions of figure 6 to solutions with positive mass $|m|$ by changing $T_{0} \rightarrow-T_{0}$.

${ }^{20}$ Notice that we have chosen potentials where the fixed point exist even at arbitrary small positive values of $x$. For another choice of potentials where the fixed point exist only up to a positive limiting value $x_{*}$ (see section 6), the structure is expected to be the one described here at least for $x_{*}<x<x_{c}$, and the structure in the region $x \leq x_{*}$ can be analyzed numerically.
} 
will label, by an integer $n=1,2, \ldots$, with $n=1$ being the rightmost solution in the figure (the one having the maximum $T_{0}$ ).

Understanding of the qualitative shape of the left figure 6 is given by the fact that for $x<x_{c}, T \ll 1$ and $\lambda$ near the fixed point value $\lambda_{*}$, the approximate solution of the tachyon is given by $T \sim r^{2} \sin [k \log r+\phi]$. Note that the constant $k$ is fixed for fixed $x$ but the normalization and $\phi$ are determined by the boundary conditions and IR regularity. Therefore the tachyon starts at the boundary, evolves into the sinusoidal form for a while, and then at end diverges. Different solutions differ in the region in which they are sinusoidal, and it is this region that controls their number of zeros. This is explained in more detail in appendix $\mathrm{F}$.

For the solutions with high label $n$, the tachyon changes sign several times before diverging in the IR. As we move to the left toward the vertical blue line in figure 6 (left), a new zero in the tachyon solution appears every time the mass curve crosses the horizontal axis. For $m=0$ we expect an infinite number of regular solutions for all positive integers $n \geq 1$. The presence of several such solutions reflect the violation of the BF bound, and are reflecting the Efimov minima seen in other contexts (see $[7,10,11])$. This agrees also with similar recent observations in [15]. The mass $m_{n}$ below which there are $n$ regular solutions (from figure 6) scales as $m_{n} \sim e^{-\frac{2 \pi n}{k}}$ with $k$ a constant given in (F.2), as shown in appendix F.

The hint of such multiple regular solutions/saddle points was seen already in [101, 102] that treated the flavor sector in the quenched approximation. Indeed, a second regular solution was seen beyond the dominant one. In that case a calculation of the spectrum of mesons in this second solution indicated that this saddle point was unstable, as the spectra were tachyonic.

It is interesting to point out that the presence of the Efimov-like tower of regular solutions is not tied uniquely to the existence of an IR fixed point solution in the landscape of the bulk theory that violates the BF bound. Even modifications of the potentials that do not allow this IR fixed point may still have the Efimov tower. The qualitative reason is that once the bulk theory has a regime that is near critical, this is enough to trigger the presence of such multiple saddle points. This is indeed the case in $[101,102]$ where in the quenched approximation a second solution exists even though there are no such IR fixed point. We have checked that exactly the same happens here in the probe limit $x \rightarrow 0$, where the fixed point is absent, if the potentials of scenario I are used. Moreover, for the scenario II the full Efimov tower remains even in the probe limit.

There is a more detailed analysis of the regular solutions in appendix F. The comparison of the free energies of the various regular solutions is made in section 9.

\subsection{Background solutions at vanishing quark mass}

To summarize the results of the above analysis, we identified the solutions of the contoured region of figure 5 as the physical ones. For $x<x_{c}$ we found solutions for all $m \geq 0$, whereas for $x \geq 0$ we found that $m>0$ so that the solution for $m=0$ was absent. 
We will discuss now how the background solutions vary as we move around figure 5 (left). We will start with the massless case, where also an additional special background exist (for all $x$ ), for which the tachyon is identically zero.

\subsubsection{Solution with identically vanishing tachyon}

Let us start by analyzing the special solution with vanishing tachyon, which is not included in figure 5. In the absence of the tachyon, the system is otherwise the one studied in [71-73], but with the dilaton potential $V_{g}$ replaced by $V_{\text {eff }}=V_{g}-x V_{f 0}$. This potential is guaranteed to have a maximum, corresponding to an IR fixed point, in the Banks-Zaks region since we matched it with the QCD $\beta$-function. For the choice of eqs. (8.24)-(8.28) the fixed point actually exists for all $0<x<11 / 2 .^{21}$

There is a single solution to the equations of motion that reaches the IR fixed point, described in section E.3 of appendix E. It is similar to the backgrounds studied in [86] where a $\beta$-function inspired by supersymmetry was used. Similar backgrounds were also studied at finite temperature in [87]. We identify this special solution as the background corresponding to the chiral symmetry conserving phase, as the vev $\sigma$ vanishes. It is easy to construct the background numerically by shooting from the vicinity of the IR fixed point, e.g., by using the expansions of appendix E.3.

We plot the background for $x=2$ and 4 in figure 7, where we fixed the scale $R$ of the IR expansions (E.48) and (E.49) to one. The geometry is expected to asymptote to AdS both in the UV and in the IR, reflecting the flow from the IR fixed point to the standard UV one. Actually $A$ is very closely linear in $\log r$ for all $r$, so that the deviation from AdS is not visible in the plots. Similar observation was made in [86]. While the solution with vanishing tachyon exist for all $x$, we will show in the next section that the other massless solution which involves a nontrivial tachyon profile and therefore chiral symmetry breaking (red dashed line in figure 5) has lower free energy whenever it exist, i.e., for $x<x_{c}$. Therefore, this background which correspond to a field theory flowing to an IR fixed point, is the physical one (for massless quarks) only for $x \geq x_{c}$, and $x_{c}$ is indeed the edge of the conformal window.

\subsubsection{Solutions having nontrivial tachyon dependence}

The backgrounds having vanishing quark mass lie on the red dashed line in figure 5 . We plot the corresponding background as a function of $r$ for a few values of $x$ in figure 8 . As we matched with the IR expansions and chose their scale $R$ to be unity, the IR scale is approximately fixed to $\mathcal{O}(1)$. The changing of the background as the critical value $x_{c} \simeq 3.9959$ is approached, is best visible in the solutions of $\lambda$ (the solid red curves).

The dependence of the solutions on $x$ meets the expectations from field theory. For $x=2$ the solution is "running": $\lambda$ has simple and smooth dependence on $\log r$. As $x$ is increased to 3 , a small distortion appears which becomes better visible for $x=3.5$. The solution of $\lambda$ is developing a plateau at $\lambda \simeq 25$, as it approaches a fixed point. Indeed for

\footnotetext{
${ }^{21}$ This is also the case for the potential associated to scenario II, described in appendix C. We could also slightly modify the potential so that the fixed point, disappears for $x<x_{*}<x_{c}$. We have also analyzed such a case and find only minor differences from those analyzed in detail in this paper.
} 

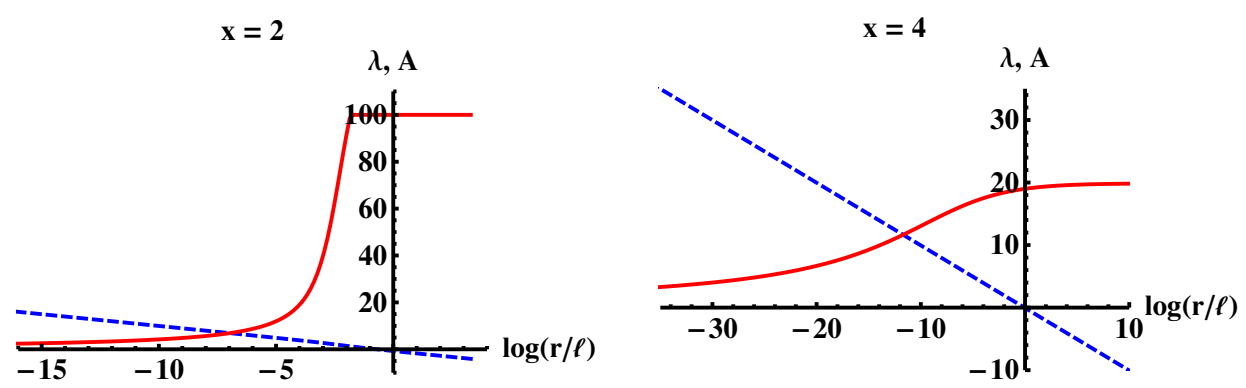

Figure 7. The backgrounds with identically vanishing tachyon for $x=2$ (left) and $x=4$ (right). The red solid, and blue dashed curves are the values of $\lambda$, and $A$ as functions of $\log (r / \ell)$, respectively.
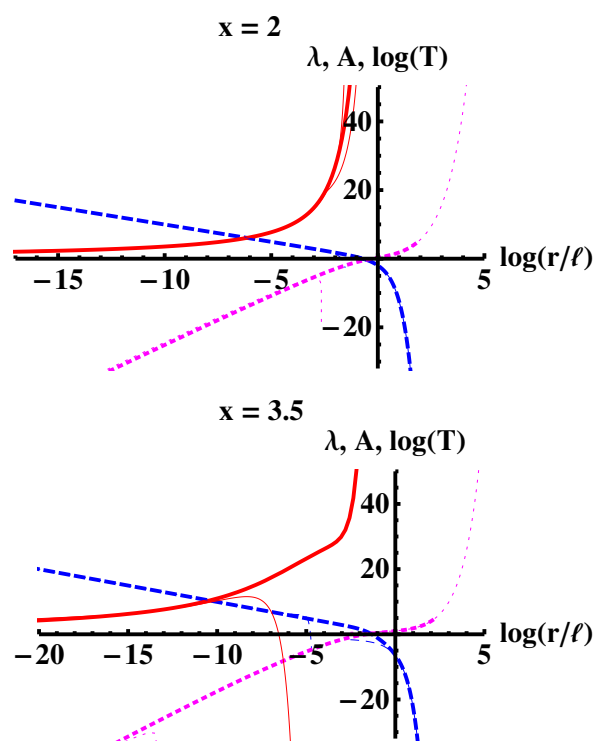

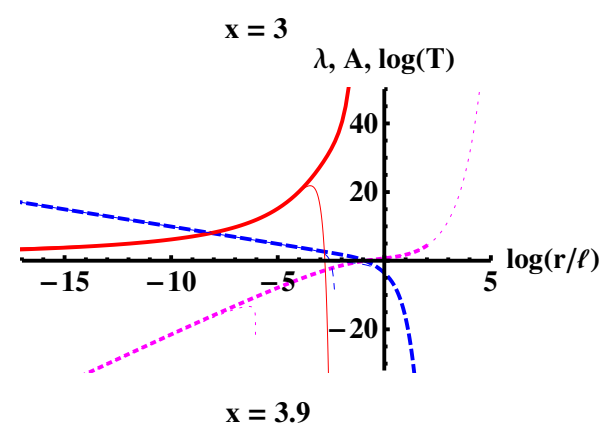

$\lambda, \mathrm{A}, \log (\mathrm{T})$

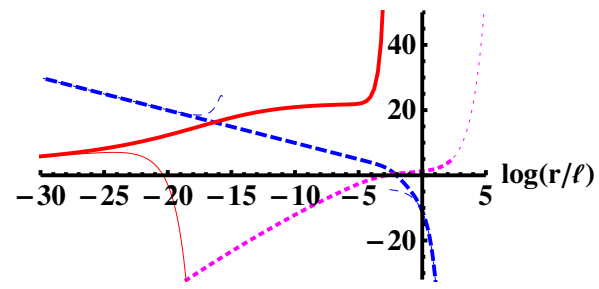

Figure 8. The background for vanishing quark mass for various values of $x$ (see the labels). The red solid, blue dashed, and magenta dotted curves are the values of $\lambda, A$, and $\log T$ as functions of $\log (r / \ell)$, respectively. The thin lines are the UV and IR expansions of the solutions.

$x=3.9$ the coupling constant $\lambda$ "walks": it takes an approximately constant value as $r$ changes by a few orders of magnitude. Such walking backgrounds have been studied in the context of IHQCD by using a model $\beta$-function in the probe limit in $[88,89]$.

We also note that $A$ (dashed blue curves) depends linearly on $\log r$ up to the IR region so that approximately $A \simeq-\log (r / \ell)$, and the metric is thus very close to the AdS one, even over the quasiconformal region where $\lambda$ walks. The tachyon (dotted magenta curves) is small and decoupled from the evolution of $A$ and $\lambda$ in the UV and in the walking region. $^{22}$ It becomes $\mathcal{O}(1)$ (the curve crosses zero) only after the coupling has already

\footnotetext{
${ }^{22}$ It is difficult to obtain solutions with the value of mass close enough to zero to produce the (quadratically vanishing) tachyon dependence of the massless solutions when shooting from the IR due to limitations from numerical precision. Therefore, we matched the background solution at $\log r \sim-8$ with a tachyon solution that was obtained by shooting from the UV and assuming decoupling, and plotted a combination of these to obtain the truly massless tachyon profile. A similar procedure was required for figure 11 below.
} 
started to diverge. This agrees with our expectation that the UV behavior, the behavior in the walking region, and in particular the phase structure is basically independent of the choices of IR behaviors of the potentials and the form of the tachyon action for large $T$.

We also show the UV and IR expansions of the various fields, given in eqs. (D.2), (D.9) of appendix D, and in eqs. (8.29), respectively, as thin lines where possible. These lines are often poorly visible since they overlap with the background. In the running region $(x=2)$ the full solution can be obtained to a good approximation by interpolating between the expansions. As $x$ increases, the region of validity of the UV expansions is pushed to smaller $\log r$, and neither UV nor IR expansions work in the walking region that grows as $x \rightarrow x_{c}$.

It is also illustrative to discuss the behavior of the system in terms of the $\beta$-functions. For this we recall that in the absence of the tachyon, potentials and $\beta$-functions are linked according to eqs. (8.18), and (8.19). By using this fact we can quantitatively estimate the validity of the tachyon decoupling in the UV and in the IR that was discussed above. First, recall that $V_{f}(\lambda, T)$ vanishes exponentially for large $T$. Therefore, in accordance with the discussion of section 6 , the behavior of $\lambda$ and $A$ is described by $V_{g}(\lambda)-x V_{f 0}(\lambda)\left(V_{g}(l)\right)$ in the UV (IR) where $T \rightarrow 0(T \rightarrow \infty)$. Eq. (8.19) gives directly the approximate $\beta$-function in the $\mathrm{UV}$, whereas in the IR we must replace $V_{\text {eff }}(\lambda) \rightarrow V_{g}(\lambda)$ as in the Yang-Mills case [71-73].

We show in figure 9 the $\beta$-functions corresponding to the UV (dashed blue curves) and IR (dotted magenta curves) potentials, obtained by solving eq. (8.19), and compare them to $d \lambda / d A$ evaluated along the RG flow of the numerical solution (red solid curves) for various values of $x$. First, notice that the $x$ dependence of the effective $\beta$-function $d \lambda / d A$ is as expected from figure 8: for $x=2$ it is qualitatively similar to the Yang-Mills $\beta$-function, and as $x$ approaches $x_{c}$ we find a typical quasiconformal behavior where the fixed point is almost reached at a finite value of the coupling.

As $\lambda \rightarrow 0$ the effective $\beta$-function $d \lambda / d A$ matches very well with the expectation from the tachyon decoupling (the dashed blue curves). Similarly, toward the IR $(\lambda \rightarrow \infty)$ the asymptotics of the red curves are similar to the magenta ones, which were obtained by taking $\lambda \rightarrow \infty$. However the convergence towards the decoupling limit (blue curves in the UV, and magenta ones in the IR) is slower in the IR than in the UV. The main reason for this is understood by studying figure 8 . Since we plot the $\beta$-functions as functions of the coupling $\lambda$, and $\lambda$ diverges in the IR much faster than the tachyon, the tachyon decoupling as $\lambda \rightarrow \infty$ takes place slowly. This is confirmed in figure 10 by plotting the factor $\exp \left(-a T^{2}\right)$ of the tachyon potential, which controls the tachyon decoupling, as a function of $\lambda$. Indeed this factor approaches the constant value of one quickly in the UV, whereas the convergence to zero in the IR is slower.

Notice the clear similarity in the $\lambda$ dependencies of this factor and the $\beta$-function $d \lambda / d A$ in figure 9 .

Finally we plot the effective $\gamma$-function

$$
\frac{\gamma}{T}=\frac{1}{T} \frac{d T}{d A}=\frac{d \log T}{d A}
$$

along the RG flow against $\lambda$ and $\log r$ in figure 11. When the quark mass is zero, $\gamma / T$ approaches -3 in the deep UV (see appendix D.1.1). In the UV region $\gamma / T$ is approximately 

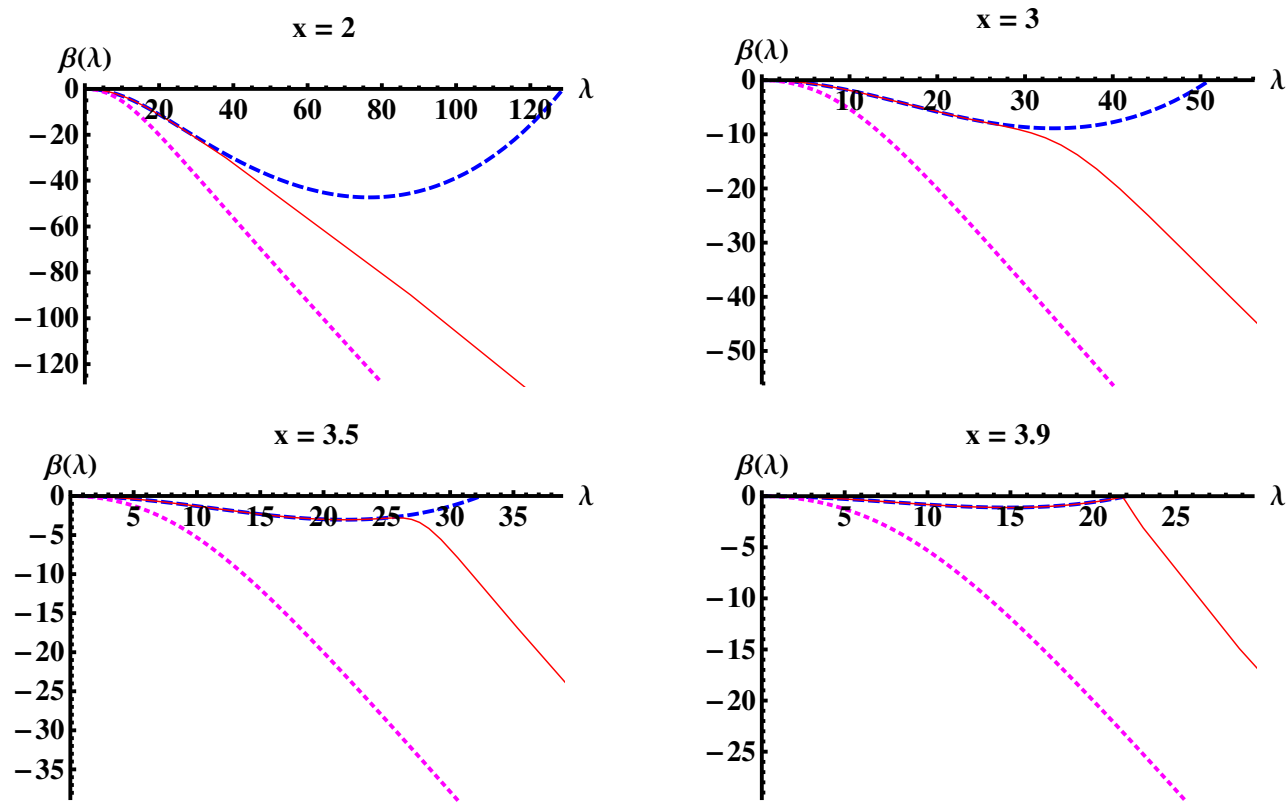

Figure 9. The $\beta$-functions for vanishing quark mass for various values of $x$ (see the labels). The red solid, blue dashed, and magenta dotted curves are the $\beta$-functions corresponding to the full numerical solution $(d \lambda / d A)$ along the $\mathrm{RG}$ flow, the potential $V_{\text {eff }}=V_{g}-x V_{f 0}$, and the potential $V_{g}$, respectively.

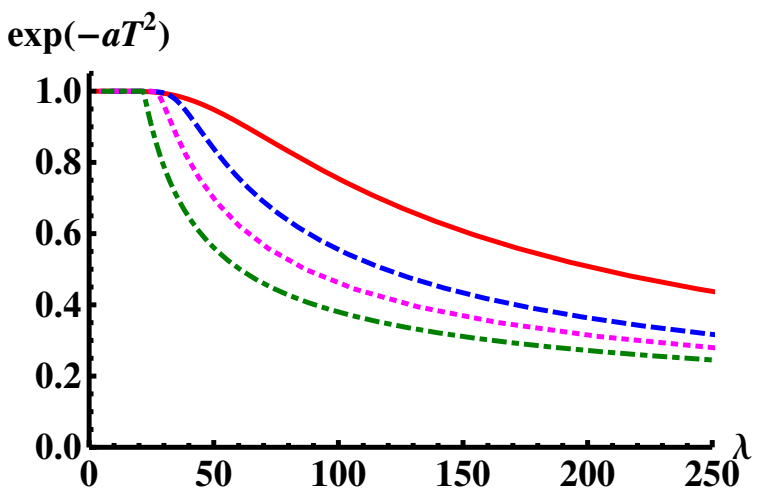

Figure 10. The factor $\exp \left(-a T^{2}\right)$ plotted against $\lambda$ along the RG flow for various values of $x$. The red solid, blue dashed, magenta dotted, and green dotdashed curves have $x=2,3,3.5$, and 3.9, respectively.

independent of $x$ and increases with $\lambda$ until it reaches -2 near the value $\lambda \simeq \lambda_{c}$ where the fixed point develops as $x \rightarrow x_{c}$. This is in line with the discussion of the preceding sections. In particular, when plotted as a function of $\log r$ we see that $\gamma / T$ is close to -2 in the walking region, corresponding to the saturation of the BF bound. We have checked that this behavior gets more pronounced as we choose values of $x$ even closer to $x_{c}$ so that a plateau near the value -2 develops in the left hand plot of figure 11 . 

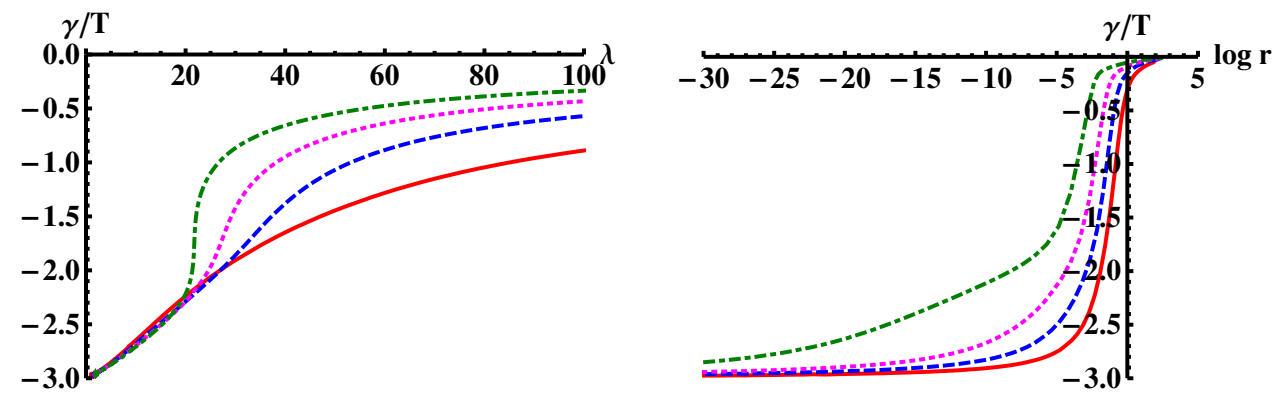

Figure 11. The $\gamma$-functions along the RG flow for vanishing quark mass as a function of $\lambda$ (left) and $\log r$ (right) for various values of $x$. The red solid, blue dashed, magenta dotted, and green dotdashed curves have $x=2,3,3.5$, and 3.9 , respectively.

\subsection{Backgrounds at generic quark masses}

We now discuss the solutions of figure 5 for generic quark masses. As pointed out above, the massive solutions (the contoured region left of the blue and dashed red lines in figure 5) exist for all values of $x$. Except for the modified UV asymptotics of the tachyon, no new classes of qualitatively different backgrounds with respect to the massless case are found.

However, adding a mass introduces a new scale to the system, which affects the background in a different way depending on its size and the value of $x .^{23}$ This effect may be illustrated by studying the ratio of the UV and IR scales of the background, which measures how close to the IR fixed point the system comes. We define the scales in term of the UV and IR expansions, i.e., $\Lambda_{\mathrm{UV}}=\Lambda$ in eqs. (D.2) and $\Lambda_{\mathrm{IR}}=1 / R$ in eqs. (8.29), which we have fixed to one.

The dependence of $\Lambda_{\mathrm{UV}} / \Lambda_{\mathrm{IR}}$ on the quark mass in UV units $m / \Lambda_{\mathrm{UV}}$ and on $x$ is depicted in figure 12, and meets the expectations from field theory. The left-hand plot shows the ratio as a function of $x$ for various choices for the quark mass. We see that there is a qualitative difference between the regions with $x<x_{c}$ and $x>x_{c}$. For $x<x_{c}$, chiral symmetry breaks spontaneously even for $m=0$, and there is some range of small masses where the background is essentially independent of $m$. This is best seen on the right hand plot, where $\Lambda_{\mathrm{UV}} / \Lambda_{\mathrm{IR}}$ levels for small masses for values of $x$ below the critical line (lowest curves).

When the mass grows large enough (essentially larger than the scale of the spontaneous symmetry breaking), it starts to fix the IR scale directly, and $\Lambda_{\mathrm{UV}} / \Lambda_{\mathrm{IR}}$ decreases. For $x>x_{c}$ there is no spontaneous chiral symmetry breaking, and the IR scale is determined smoothly by the value of the mass. From the log-log plot on the right we see that the dependence of $\Lambda_{\mathrm{UV}} / \Lambda_{\mathrm{IR}}$ on $m$ is a power-law. Naively one could expect that the IR scale is directly given by the quark mass, $m \sim \Lambda_{\mathrm{IR}}$, so that $\Lambda_{\mathrm{UV}} / \Lambda_{\mathrm{IR}} \propto\left(m / \Lambda_{\mathrm{UV}}\right)^{-1}$ (which is also the result one gets by approximating $T(r) / \ell=m r$ and using the arguments of appendix $F$ ). The nontrivial energy dependence of the quark mass modifies the power from -1 to larger values, as depicted in figure 4 (right).

\footnotetext{
${ }^{23}$ See $[119,120]$ for an analysis within a different framework.
} 

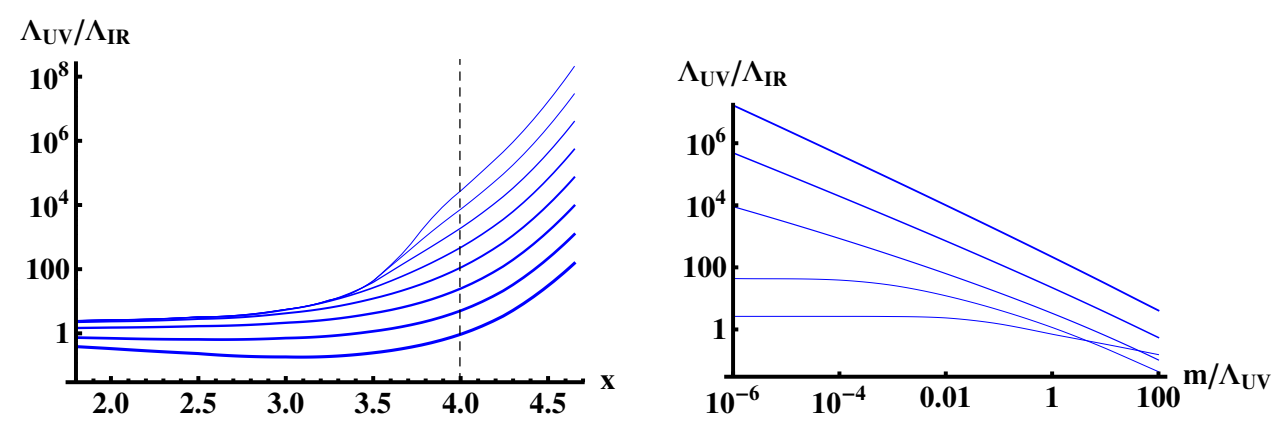

Figure 12. Left: The ratio of the UV $\left(\Lambda_{\mathrm{UV}}\right)$ and IR $\left(\Lambda_{\mathrm{IR}}\right)$ scales as a function of $x$ for $m / \Lambda_{\mathrm{UV}}=$ $10^{-6}, 10^{-5}, \ldots, 10$ from thin to thick lines (top to bottom). The vertical dashed line is at critical $x_{c} \simeq 3.9959$. Right: The ratio of the UV and IR scales as a function of $m / \Lambda_{\mathrm{UV}}$ for $x=2,3.5,3.9$, 4.25 , and 4.5 from thin to thick lines (bottom to top).

\section{The free energy}

We now analyze the free energy for zero quark mass. In this case we identified two distinct solutions, one with identically vanishing tachyon and the other with nontrivial tachyon background. We shall show that the latter one is energetically favorable in the region where it exists $\left(x<x_{c}\right)$. We start with the generic definition of free energy for our action.

The free energy is given by the on-shell Euclidean action plus counterterms (which we will not need in this article). From (5.5) the Euclidean action takes the form:

$$
\begin{aligned}
\mathcal{S}= & -\left(M^{3} N^{2}\right) \int d^{5} x\left[\sqrt{g}\left(R-\frac{4}{3} \frac{(\partial \lambda)^{2}}{\lambda^{2}}+V_{g}(\lambda)\right)\right. \\
& \left.-x V_{f}(\lambda, T) \sqrt{\operatorname{det}\left(g_{a b}+h(\lambda, T) \partial_{a} T \partial_{b} T\right)}\right] .
\end{aligned}
$$

By using the Einstein equations (5.7) we can eliminate $R$, which leads to

$$
\begin{aligned}
\mathcal{S}_{\mathrm{os}}= & -\left(M^{3} N^{2}\right) \int d^{5} x\left[-\frac{2}{3} e^{5 A} V_{g}(\lambda)+\frac{2}{3} x e^{5 A} V_{f}(\lambda, T) \sqrt{1+e^{-2 A} h(\lambda, T) T^{\prime 2}}\right. \\
& \left.-\frac{x}{3} \frac{e^{3 A} h(\lambda, T) T^{\prime 2}}{\sqrt{1+e^{-2 A} h(\lambda, T) T^{\prime 2}}}\right] .
\end{aligned}
$$

Next, one can solve $V_{g}$ from (5.8) and (5.9):

$$
\begin{aligned}
e^{5 A} V_{g}(\lambda)= & \left(3 A^{\prime} e^{3 A}\right)^{\prime}+\frac{x}{2} e^{5 A} V_{f}(\lambda, T) \sqrt{1+e^{-2 A} h(\lambda, T) T^{\prime 2}} \\
& +\frac{x}{2} e^{5 A} \frac{V_{f}(\lambda, T)}{\sqrt{1+e^{-2 A} h(\lambda, T) T^{\prime 2}}}
\end{aligned}
$$

and inserting this into (9.3) the on-shell action can be integrated:

$$
\mathcal{S}_{\mathrm{os}}=2 M^{3} N^{2} \int d^{5} x\left(A^{\prime} e^{3 A}\right)^{\prime}=2 M^{3} N^{2} V_{4}\left[A^{\prime} e^{3 A}\right]_{\epsilon}^{r_{0}}
$$


Here $\epsilon$ and $r_{0}$ are the UV and IR cutoffs, respectively. In all the backgrounds which we consider here the contribution from the IR vanishes, so we will drop that term. This is a matter of consistency of the models as we do not in general expect contribution from IR singularities or boundaries in well defined holographic models.

Following [71-73] the Gibbons-Hawking boundary term is given by

$$
\mathcal{S}_{\mathrm{GH}}=2 M^{3} N^{2} \int_{\partial M} d^{4} x \sqrt{h} K=8 M^{3} N^{2} V_{4} A^{\prime}(\epsilon) e^{3 A(\epsilon)},
$$

where we have used that [71-73] $K=4 e^{-A} A^{\prime}$. We obtain the following simple and general expression for the free energy of the system:

$$
\mathcal{E}=6 M^{3} N^{2} V_{4} A^{\prime}(\epsilon) e^{3 A(\epsilon)} .
$$

\subsection{The free energy difference of the $m=0$ backgrounds}

The free energy calculated above is obviously divergent as $\epsilon \rightarrow 0$, and needs to be regularized. However, different solutions with the same UV boundary conditions (the quark mass $m$ and the UV scale $\Lambda_{\mathrm{UV}}$ ) have the same divergent terms as well as counterterms and differ only through a finite term which can be extracted from the UV expansions. This is the case in particular for the two backgrounds having zero quark mass, one with vanishing tachyon and the other with a nontrivial tachyon solution. we now discuss in detail how the free energy difference between these two backgrounds can be obtained, by expanding all quantities as series at the UV singularity $r=0$. Since the leading UV free energy behaves as $1 / \epsilon^{4}$, corrections $\mathcal{O}\left(r^{4}\right)$ to the behavior of $A$ and $\lambda$ will possibly contribute to the finite terms.

As discussed above, in the UV the tachyon decouples from the equations of motion for $A$ and $\lambda$. For $m=0$, the leading corrections to these equations due to the tachyon are suppressed by $T^{2}$ or $e^{-2 A} T^{2}$, i.e., by $\mathcal{O}\left(r^{6}\right)$. Therefore the coupling to tachyon does not affect the free energy directly in the massless case, and we can set it to zero.

Let us take

$$
V_{\mathrm{eff}}(\lambda)=V_{g}(\lambda)-x V_{f}(\lambda, 0)=V_{g}(\lambda)-x V_{f 0}(\lambda)=\frac{12}{\ell^{2}}\left[1+V_{1} \lambda+V_{2} \lambda^{2}+\cdots\right] .
$$

The finite contribution to the free energy can be studied by writing

$$
\begin{aligned}
A(r) & =A_{0}(r)+r^{4} A_{1}(r)+\mathcal{O}\left(r^{6}\right) \\
\lambda(r) & =\lambda_{0}(r)+r^{4} \lambda_{1}(r)+\mathcal{O}\left(r^{6}\right)
\end{aligned}
$$

in close analogy to eqs. (3.9), where $A_{i}(r)$ and $\lambda_{i}(r)$ have now series expansions in $1 / \log r$ at $r \rightarrow 0$. The expansions of for $A_{0}$ and $\lambda_{0}$ are given in eqs. (D.2). Inserting these as well as suitable Ansätze for $A_{1}$ and $\lambda_{1}$ in the EoMs of $A$ and $\lambda$, and expanding up to $\mathcal{O}\left(r^{4}\right)$ we find

$$
\begin{aligned}
& A_{1}(r)=\hat{A}\left[1-\frac{19}{12 \log (r \Lambda)}+\cdots\right] \\
& \lambda_{1}(r)=\frac{\hat{A}}{V_{1}}\left[-5+\frac{445 V_{1}^{2}-320 V_{2}}{36 V_{1}^{2} \log (r \Lambda)}+\cdots\right]
\end{aligned}
$$


where $\Lambda$ is the same UV scale that appears in the expansions of $A_{0}$ and $\lambda_{0}$, and $\hat{A}$ is a free parameter. It is recognized as an integration constant of the EoMs that did not appear in the leading order $\left(\mathcal{O}\left(r^{0}\right)\right)$ UV expansions. Two solutions having the same $\Lambda$ and $m=0$, but different IR behavior, will have different $\hat{A}$ as its value is not fixed by the EoMs asymptotically in the UV. Inserting the results for $A_{1}$ and $\lambda_{1}$ in the expression (9.6) for the free energy, we find ${ }^{24}$

$$
\Delta \mathcal{E}=15 M^{3} N^{2} V_{4} \ell^{3} \Delta \hat{A},
$$

where $\Delta \mathcal{E}$ and $\Delta \hat{A}$ are the differences between the two solutions in free energy and the constant $\hat{A}$, respectively.

It is useful to also study the corresponding variation in the $\beta$-function. We will actually use the phase variable defined by

$$
X(r)=\frac{\lambda^{\prime}(r)}{3 \lambda(r) A(r)}=\frac{\beta}{3 \lambda}
$$

We may again write

$$
X(r)=X_{0}(r)+r^{4} X_{1}(r)+\mathcal{O}\left(r^{6}\right) .
$$

Substituting the expansions (9.8), (9.9) in the definition of $X$, we identify

$$
X_{1}(r)=\hat{A}\left[-\frac{15}{2} \log (r \Lambda)+\frac{5\left[80 V_{1}^{2}-64 V_{2}-\log [-\log (r \Lambda)]\left(23 V_{1}^{2}-64 V_{2}\right)\right]}{24 V_{1}^{2}}+\cdots\right] .
$$

By using the logarithmic expansion from above, the result can be expressed in terms of $\lambda$ :

$$
X(\lambda)=X_{0}(\lambda)+\frac{20 \hat{A}}{3 V_{1} \Lambda^{4}} \exp \left[\frac{\log \left(9 V_{1} / 8\right)\left(23 V_{1}^{2}-64 V_{2}\right)}{9 V_{1}^{2}}\right] e^{-\frac{32}{9 V_{1} \lambda}} \lambda^{\frac{14}{9}-\frac{64 V_{2}}{9 V_{1}^{2}}}[1+\mathcal{O}(\lambda)] .
$$

Finally, we recall that when the tachyon has decoupled, $X$ satisfies

$$
\lambda X^{\prime}(\lambda)=\left[8+\frac{3 \lambda}{X(\lambda)} \frac{d}{d \lambda} \log V_{\text {eff }}(\lambda)\right] \frac{X(\lambda)^{2}-1}{6} .
$$

Indeed it is easy to check that the result (9.14) is consistent with this equation, and that $\hat{A}$ is the integration constant which parametrizes all solutions of the differential equation for given potential $V$.

The remaining task is to extract the coefficients $\hat{A}$ from the chiral symmetry breaking (with nontrivial tachyon profile) and conserving backgrounds (with identically vanishing tachyon) which have zero quark mass, and then use the formula (9.10) to calculate the free energy difference. Extraction of the coefficients is done by studying the variation $X_{1}$ of the phase function, and details are given in appendix G.1.

We find that the chiral symmetry breaking solution is the energetically favorable one in the region where it exists, i.e., for $0<x<x_{c}$. We plot the free energy difference (setting $M^{3} N^{2} V_{4}=1$ ) in figure 13 (solid blue curve).

\footnotetext{
${ }^{24}$ In order to obtain the correct numerical factor 15 in eq. (9.10) one needs to follow carefully the regularization procedure detailed in [76]. Even though their analysis assumes a single scalar field it applies here without changes since the extra field, the tachyon, is negligibly small in the UV.
} 


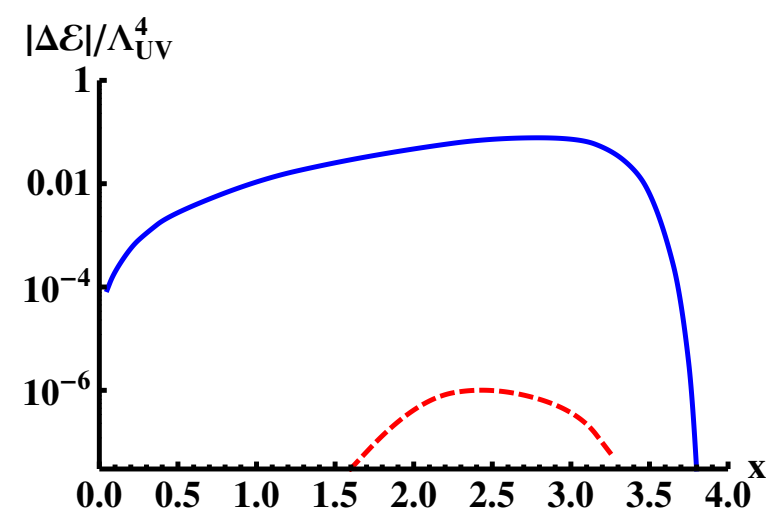

Figure 13. The free energy difference between the chiral symmetry conserving $(T \equiv 0)$ and breaking $(T \rightarrow \infty$ as $r \rightarrow \infty)$ solutions. Solid blue curve is the difference for the chiral symmetry breaking solution having a monotonic tachyon with no zeroes, and the dashed red curve is the difference for the solution having one zero.

Notice that $\Delta \mathcal{E}$ approaches zero both as $x \rightarrow 0$ and as $x \rightarrow x_{c}$. In the case $x \rightarrow 0$, we do expect that the effect vanishes as indeed the number of flavors, which controls the backreaction of the tachyon, vanishes. One expects linear dependence $\Delta \mathcal{E} \propto x$ : since the background configurations behave smoothly as $x \rightarrow 0$, the energy difference arises due to the explicit $x$ dependence in the action and due to its linear effect on the background. The case $x \rightarrow x_{c}$ will be discussed in the next section. For $x_{c}<x<5.5$ only one solution with zero quark mass exists (the one with $T \equiv 0$ ), as discussed in section 8 , so there is no need for comparison.

There are also solutions with zero quark mass where the tachyon has one or more zeroes (see appendix F). We have verified that these solutions have larger free energies than the one without a tachyon zero. The red dashed line in figure 13 is the free energy difference between the background with vanishing tachyon and the one with a nontrivial tachyon solution having one zero. The energy difference between the $T \equiv 0$ solution and the solutions having more than one tachyon zeroes is even smaller.

Therefore, for $x<x_{c}$ and $m=0$, the standard tachyon solution has the lowest free energy and the chirally symmetric one, $T=0$, has the largest free energy. All other undulating solutions have free energies that are between these two (and closest to the $T=0$ solution).

For $|m|>0$, the standard tachyon solution has the lowest free energy and the nonstandard ones, have higher free energy.

\section{Scaling below the conformal window}

\subsection{BKT scaling of the chiral condensate}

We shall now argue that the chiral condensate $\propto \sigma$ obeys the BKT [8] or Miransky [9] scaling behavior,

$$
\sigma \propto \exp \left(-\frac{c}{\sqrt{x_{c}-x}}\right)
$$


as $x$ approaches the critical value $x_{c}$ where the solution ceases to exist. This argument will be supported with numerical results in section 10.3. The ratio of the UV and the IR energy scales will show similar scaling. This behavior is known to arise both in DysonSchwinger [9, 38] and holographic approaches [7, 12-15, 88, 89]. Indeed, our analysis has many similarities with both Dyson-Schwinger and earlier holographic approaches, and in particular with the recent study [15] of a related model.

We shall not give a precise proof but only sketch how the scaling arises. It is enough to study the region near the UV where the tachyon is small $T(r) \ll 1$, so the tachyon decouples from the EoMs of $\lambda$ and $A$. We will neglect the logarithmic corrections to the tachyon (see appendix D.1.1) which play no role in the scaling argument.

In the deep UV, where the coupling is small $\lambda \ll 1$, the tachyon behaves as

$$
T(r) \sim \sigma r^{3} .
$$

As $r$ increases $T$ stays small, and $\lambda$ starts to approach the fixed point value $\lambda=\lambda_{*}$ which maximizes $V_{g}(\lambda)-x V_{f 0}(\lambda)$. The behavior of $\lambda$ and $A$ in this region is given by the $T=0$ asymptotics of section E.3:

$$
\begin{aligned}
& \lambda=\lambda_{*}+\mathcal{O}\left[\left(\frac{r}{r_{\mathrm{UV}}}\right)^{-\delta}\right] \\
& A=-\log \left(r-r_{0}\right)+A_{0}+\mathcal{O}\left[\left(\frac{r}{r_{\mathrm{UV}}}\right)^{-2 \delta}\right]
\end{aligned}
$$

where $\delta$ is a positive parameter defined in eq. (E.51). This approximation is valid for some intermediate region $r_{\mathrm{UV}} \ll r \lesssim r_{\mathrm{IR}}$, where at the scale $r_{\mathrm{IR}}$ the tachyon finally becomes $\mathcal{O}(1)$, and drives the system away from the IR fixed point. We choose $x$ near the critical value, $0<x_{c}-x \ll 1$. The tachyon IR mass was calculated above in section 8 :

$$
\Delta_{\mathrm{IR}}\left(4-\Delta_{\mathrm{IR}}\right)=-m_{\mathrm{IR}}^{2} \ell_{\mathrm{IR}}^{2}=G\left(\lambda_{*}, x\right),
$$

where

$$
G(\lambda, x) \equiv \frac{24 a(\lambda)}{h(\lambda)\left(V_{g}(\lambda)-x V_{f 0}(\lambda)\right)} .
$$

Keeping formally $x$ fixed while varying $\lambda_{*}$, the right hand side will become equal to four as $\lambda_{*}$ reaches a critical value $\lambda_{c}: G\left(\lambda_{c}, x\right)=4$. The scaling behavior will appear as $\lambda_{*} \rightarrow \lambda_{c}$ from above, and then also $x \rightarrow x_{c}$. We expand around this point:

$$
\Delta_{\mathrm{IR}}\left(4-\Delta_{\mathrm{IR}}\right)=G\left(\lambda_{*}, x\right)=4+\frac{\partial}{\partial \lambda} G\left(\lambda_{c}, x\right)\left(\lambda_{*}-\lambda_{c}\right)+\cdots \equiv 4+\kappa\left(\lambda_{*}-\lambda_{c}\right)+\cdots .
$$

For $0<\lambda_{*}-\lambda_{c} \ll 1$, we obtain

$$
\Delta_{\mathrm{IR}} \simeq 2 \pm i \sqrt{\kappa\left(\lambda_{*}-\lambda_{c}\right)} .
$$

The tachyon solution becomes

$$
T(r) \simeq C_{\mathrm{fp}}\left(\frac{r}{r_{\mathrm{UV}}}\right)^{2} \sin \left(\sqrt{\kappa\left(\lambda_{*}-\lambda_{c}\right)} \log \frac{r}{r_{\mathrm{UV}}}+\phi\right)
$$


When $\lambda$ moves away from the fixed point, while moving towards the UV, it will at some point become smaller than $\lambda_{c}$ so that the asymptotic solution (10.9) fails. However, as we shall see, the leading scaling originates from the region where $\lambda_{*}-\lambda \ll \lambda_{*}-\lambda_{c}$, and we need the solution for smaller $\lambda$ only to make contact with the deep UV behavior and the definition of $\sigma$. Even for $\lambda \lesssim \lambda_{c}, \kappa\left(\lambda_{c}-\lambda\right)$ is small for almost the whole range of $r$ with $r_{\mathrm{UV}}<r$. Therefore, within the this region, it is sufficient to neglect $\kappa\left(\lambda_{c}-\lambda\right)$ and approximate $T(r) \propto r^{2}$. Further, we introduce an intermediate scale $\hat{r}$ where $\lambda_{*}-\lambda \sim \lambda_{*}-\lambda_{c}$, and write the approximation as

$$
\begin{array}{ll}
T(r) \simeq \hat{C}\left(\frac{r}{r_{\mathrm{UV}}}\right)^{2} ; & r_{\mathrm{UV}} \ll r \lesssim \hat{r}, \quad \lambda \lesssim \lambda_{c} \\
T(r) \simeq C_{\mathrm{fp}}\left(\frac{r}{r_{\mathrm{UV}}}\right)^{2} \sin \left(\sqrt{\kappa\left(\lambda_{*}-\lambda_{c}\right)} \log \frac{r}{\hat{r}}+\hat{\phi}\right) ; & \hat{r} \lesssim r \ll r_{\mathrm{IR}}, \quad \lambda_{c} \lesssim \lambda<\lambda_{*}
\end{array}
$$

where we use $\hat{r}$ instead of $r_{\mathrm{UV}}$ as the reference value of the logarithm for later convenience.

For $r \gtrsim r_{\mathrm{IR}}$ there is no obvious way to write a good approximation for the tachyon solution. However, as we shall see, such an approximation is not necessary for finding the scaling behavior.

The scaling behavior can be found by matching the tachyon solutions in the different regions. First, we require that the solutions of eq. (10.2) and (10.10) join approximately continuously at $r \simeq r_{\mathrm{UV}}$. This gives

$$
\hat{C} \sim \sigma r_{\mathrm{UV}}^{3}
$$

Notice that $\sigma$ is not a free parameter here, but it will later be fixed by the matching procedure. Further requiring approximate continuity at $r \simeq \hat{r}$ we find $^{25}$

$$
C_{\mathrm{fp}} \sim \sigma r_{\mathrm{UV}}^{3} ; \quad \hat{\phi} \sim 1 .
$$

The remaining task is to match with the unknown IR behavior at $r \simeq r_{\mathrm{IR}}$. First, $r_{\mathrm{IR}}$ was defined as the scale where the tachyon becomes $\mathcal{O}(1)$ and drives the system away from the fixed point, so $T\left(r_{\mathrm{IR}}\right) \sim 1$, which fixes $r_{\mathrm{IR}}$ in terms of $\sigma$ :

$$
C_{\mathrm{fp}} \sim \sigma r_{\mathrm{UV}}^{3} \sim\left(\frac{r_{\mathrm{UV}}}{r_{\mathrm{IR}}}\right)^{2}
$$

Finally, we need to match the solution to $T^{\prime}\left(r_{\mathrm{IR}}\right)$, which is $\mathcal{O}\left(1 / r_{\mathrm{IR}}\right)$, since the tachyon EoM is apparently regular in this region. Notice that we must indeed fix this number to have a solution that asymptotes to the "good" singularity in the IR. The good IR asymptotics have one free parameter, the normalization of the tachyon in the IR. This is however already determined by requiring $T\left(r_{\mathrm{IR}}\right) \sim 1$. Therefore, the argument of the sine function in (10.11) is basically fixed to a given $\mathcal{O}(1)$ number at $r=r_{\mathrm{IR}}$, which gives the desired BKT scaling:

$$
\sqrt{\kappa\left(\lambda_{*}-\lambda_{c}\right)} \log \frac{r_{\mathrm{IR}}}{\hat{r}}=\mathcal{O}(1)
$$

\footnotetext{
${ }^{25}$ This matching involves a subtlety which does not affect the scaling (see appendix $\mathrm{H}$ ).
} 
so that

$$
\frac{r_{\mathrm{IR}}}{\hat{r}} \sim \exp \left(\frac{K}{\sqrt{\lambda_{*}-\lambda_{c}}}\right)
$$

with $K$ positive. Finally, we notice that the connection between $\hat{r}$ and $r_{\mathrm{UV}}$ can be obtained from (10.3) by using the definition of $\hat{r}$. This results in a power law:

$$
\frac{\hat{r}}{r_{\mathrm{UV}}} \sim\left(\lambda_{*}-\lambda_{c}\right)^{-\frac{1}{\delta}}
$$

which can be neglected as a subleading correction to the exponential scaling. Taking this into account

$$
\frac{r_{\mathrm{IR}}}{r_{\mathrm{UV}}} \sim \exp \left(\frac{K}{\sqrt{\lambda_{*}-\lambda_{c}}}\right) \sim \exp \left(\frac{\hat{K}}{\sqrt{x_{c}-x}}\right) .
$$

The inverse of this scaling result is expected to hold for any ratio of IR and UV energy scales independently of their precise definitions. By using eq. (10.14) we find the scaling results for $\sigma$ :

$$
\sigma \sim \frac{1}{r_{\mathrm{UV}}^{3}} \exp \left(-\frac{2 K}{\sqrt{\lambda_{*}-\lambda_{c}}}\right) \sim \frac{1}{r_{\mathrm{UV}}^{3}} \exp \left(-\frac{2 \hat{K}}{\sqrt{x_{c}-x}}\right) .
$$

Notice that $x_{c}$ and $\lambda_{c}$ were defined by $G\left(\lambda_{*}\left(x_{c}\right), x_{c}\right)=4$ and $G\left(\lambda_{c}, x\right)=4$, respectively, so that $\lambda_{*}=\lambda_{c}$ at $x=x_{c}$. Since $G$ is smooth in this region, $\lambda_{*}-\lambda_{c}$ could readily be replaced by $x_{c}-x$ in the results above (after rescaling $K$ ).

In the above discussion we neglected several subtleties. These issues are analyzed in appendix H. In particular, after more careful analysis, we are able to find explicit results for $K$ and $\hat{K}$ :

$$
K=\frac{\pi}{\sqrt{\kappa}}=\frac{\pi}{\sqrt{\frac{\partial}{\partial \lambda} G\left(\lambda_{c}, x\right)}} ; \quad \hat{K}=\frac{\pi}{\sqrt{-\left.\frac{d}{d x} G\left(\lambda_{*}(x), x\right)\right|_{x=x_{c}}}} .
$$

As a final remark, we stress that the above sketch was to a large extent independent of the details of the model. In particular, we did not need any information on the nonlinear terms in the tachyon EoM and on how the IR boundary conditions are fixed.

\subsection{Scaling of the free energy}

Let us then study the scaling of the free energy difference $\Delta \mathcal{E}$ between the solutions without and with chiral symmetry breaking (at zero quarks mass), which was studied numerically in section 9. Linearizing the EoMs for $A$ and $\lambda$ by writing

$$
\begin{aligned}
A(r) & =A_{0}(r)+r^{4} A_{1}(r)+\mathcal{O}\left(r^{6}\right) \\
\lambda(r) & =\lambda_{0}(r)+r^{4} \lambda_{1}(r)+\mathcal{O}\left(r^{6}\right),
\end{aligned}
$$

we related $\Delta \mathcal{E}$ with the variation of the leading coefficients of $A_{1}$ and $\lambda_{1}$ when expressed as a series in $\log r$ at $r=0$. The source of this variation is the difference in the tachyon solution, and it can be analyzed in the limit $x \rightarrow x_{c}$. The tachyon contributes corrections $\mathcal{O}\left(T^{2}\right)$ and $\left(e^{2 A}\left(T^{\prime}\right)^{2}\right)$ to the EoMs of $A$ and $\lambda$. For zero quark mass these contributions are $\mathcal{O}\left(r^{6}\right)$ in the deep UV and thus decoupled from the variations of eqs. (10.21). However, 
as we have pointed out, in the walking region $r_{\mathrm{UV}} \lesssim r \lesssim r_{\mathrm{IR}}$ the tachyon is $\mathcal{O}\left(r^{2}\right)$, and therefore the tachyon contributions $\mathcal{O}\left(r^{4}\right)$ do couple to $A_{1}$ and $\lambda_{1} \cdot{ }^{26}$ Consequently, we expect that, e.g.,

$$
r^{4} \Delta A_{1}(r) \sim T(r)^{2} ; \quad r_{\mathrm{UV}} \lesssim r \lesssim r_{\mathrm{IR}}
$$

where $\Delta A_{1}(r)$ denotes the difference in $A_{1}$ between the solutions with intact and broken chiral symmetry. We expect that to a good enough approximation, the size of $A_{1}$ equals the coefficient $\hat{A}$ defined in eq. (9.9) even in the walking region where the UV series does not converge. ${ }^{27}$ Therefore, we obtain

$$
\Delta \hat{A} \sim \sigma^{2} r_{\mathrm{UV}}^{2}
$$

and finally the scaling result for the free energy difference reads

$$
\frac{\Delta \mathcal{E}}{M^{3} N^{2} V_{4}} \sim \Delta \hat{A} \sim \sigma^{2} r_{\mathrm{UV}}^{2} \sim r_{\mathrm{UV}}^{-4} \exp \left(-\frac{4 K}{\sqrt{\lambda_{*}-\lambda_{c}}}\right) \sim r_{\mathrm{UV}}^{-4} \exp \left(-\frac{4 \hat{K}}{\sqrt{x_{c}-x}}\right) .
$$

\subsection{Comparison with numerical results}

We now compare the analytic results above to numerical solutions. In figure 14 we plot the tachyon (left) and the coupling $\lambda$ (right) as functions of $\log r$ in an extreme walking case with $x=3.992$ such that $x_{c}-x \simeq 0.004$. The numerical tachyon solution with zero quark mass (blue thick curve on the left) was obtained by gluing together the various solutions described in appendix G.2. We compare the solution to the analytic approximations of eqs. (10.2), (10.10) and (10.11), shown as thin green dotted, magenta dashed, and solid red curves, respectively. The parameters of these curves were chosen such that $\sigma$ has the extracted value (see appendix G.2), $r_{\mathrm{UV}}=1 / \Lambda_{\mathrm{UV}}$ with $\Lambda_{\mathrm{UV}}$ obtained by fitting $\lambda$ to its UV expansion, and $\hat{r}$ is the value where $\lambda$ reaches $\lambda_{c}$. The parameters $\lambda_{c}, \lambda_{*}$ and $\kappa$ were calculated directly from the potentials, and $\hat{\phi}$ was given an arbitrary small value. The agreement between the approximation and the full numerical solution is remarkably good.

We have also compared the expected scaling to the values of chiral condensate that were extracted from the background (see section 8) as detailed in appendix G.2. The results for various values of $x$ are the dots in figure 15. The solid line is a fit to the BKT scaling behavior, given by

$$
\log \frac{\sigma}{\Lambda^{3}}=8.6-\frac{6.8}{\sqrt{x_{c}-x}},
$$

which describes the data well. The analytic result of eq. (10.20) gives for the potentials used

$$
2 \hat{K}=6.10
$$

which agrees with the fitted result 6.8 within the precision of the fit. We have checked that using only a few of the data points with the highest $x$ brings the fitted valued of $\hat{K}$ closer to the analytical one.

\footnotetext{
${ }^{26}$ The next-to-leading terms of $A$ and $\lambda$ are still $\mathcal{O}\left(r^{4}\right)$ in this region since there are no sources which could change this.

${ }^{27}$ This can be verified by inserting the Ansätze (10.21) to the EoMs and studying the solutions similarly as done for $X_{1}$ in appendix G.1.
} 

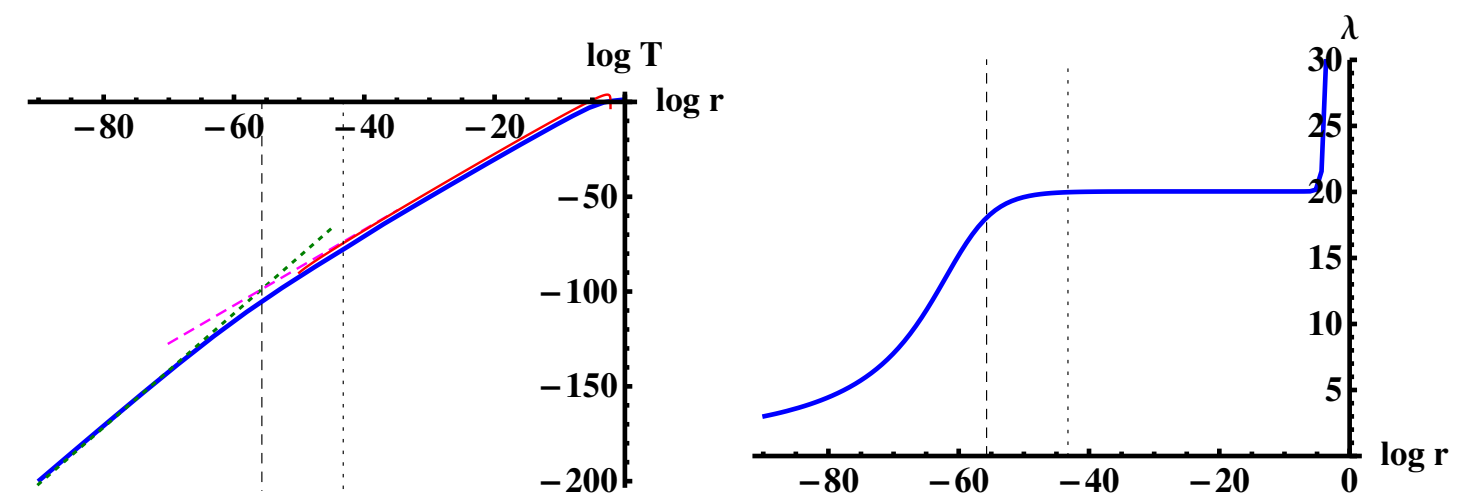

Figure 14. The tachyon $\log T$ (left) and the coupling $\lambda$ (right) as functions of $\log r$ for an extreme walking background with $x=3.992$. The thin lines on the left hand plot are the approximations used to derive the BKT scaling (see the text for explanation), and the vertical dashed (dotted) lines $\operatorname{mark} \log r_{\mathrm{UV}}(\log \hat{r})$.
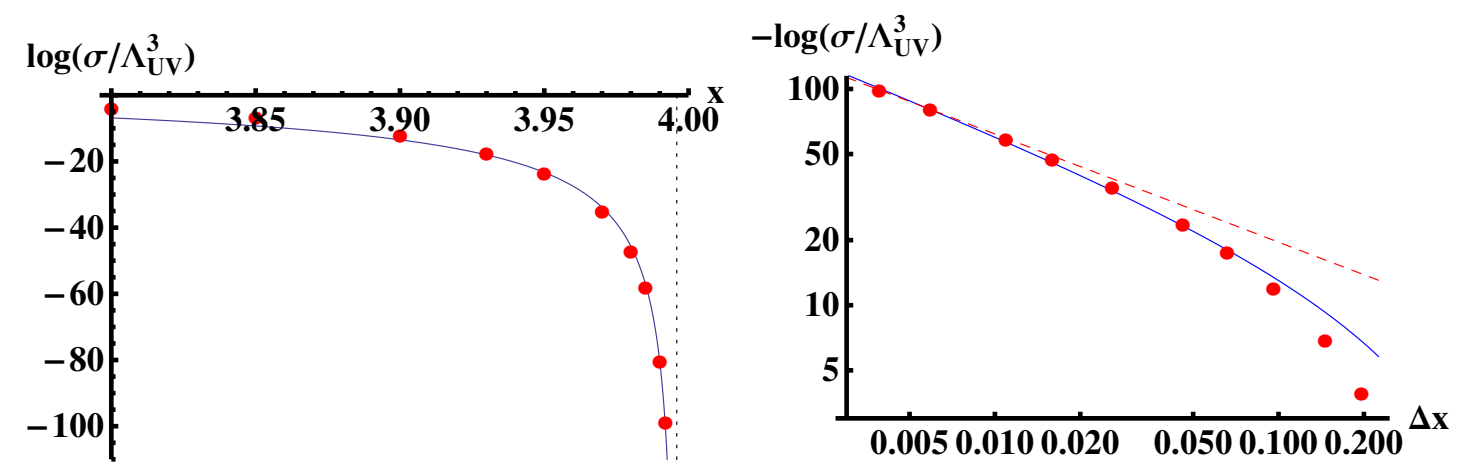

Figure 15. Left: $\log \left(\sigma / \Lambda^{3}\right)$ as a function of $x$ (dots), compared to a BKT scaling fit (solid line). The vertical dotted line lies at $x=x_{c}$. Right: the same curve on log-log scale, using $\Delta x=x_{c}-x$. To guide the eye we added the straight dashed line corresponding to the BKT scaling fit without a constant term, that was fixed to go through the data point with smallest $\Delta x$.

In figure 16 (left) we compare the ratio of the scales $\Lambda_{\mathrm{UV}}=\Lambda$ and $\Lambda_{\mathrm{IR}}=1 / R$ as defined by the UV and IR expansions of the background, respectively, to a BKT scaling fit with $\hat{K} \simeq 3.4$. We also checked that the scaling of eq. (10.14),

$$
\frac{\sigma}{\Lambda_{\mathrm{UV}}^{3}} \sim\left(\frac{r_{\mathrm{UV}}}{r_{\mathrm{IR}}}\right)^{2} \sim\left(\frac{\Lambda_{\mathrm{IR}}}{\Lambda_{\mathrm{UV}}}\right)^{2}
$$

is satisfied to a high precision on figure 16 (right).

\section{Conclusions}

We analyzed a novel class of holographic models (V-QCD), which reproduces the main features of QCD in the Veneziano limit of large $N_{f}$ and $N_{c}$ with $x=N_{f} / N_{c}$ fixed.

$\mathrm{V}-\mathrm{QCD}$ is on one hand based on a successful holographic model of Yang-Mills (YM) theory, and termed improved holographic QCD (IHQCD). IHQCD contains a dilaton 

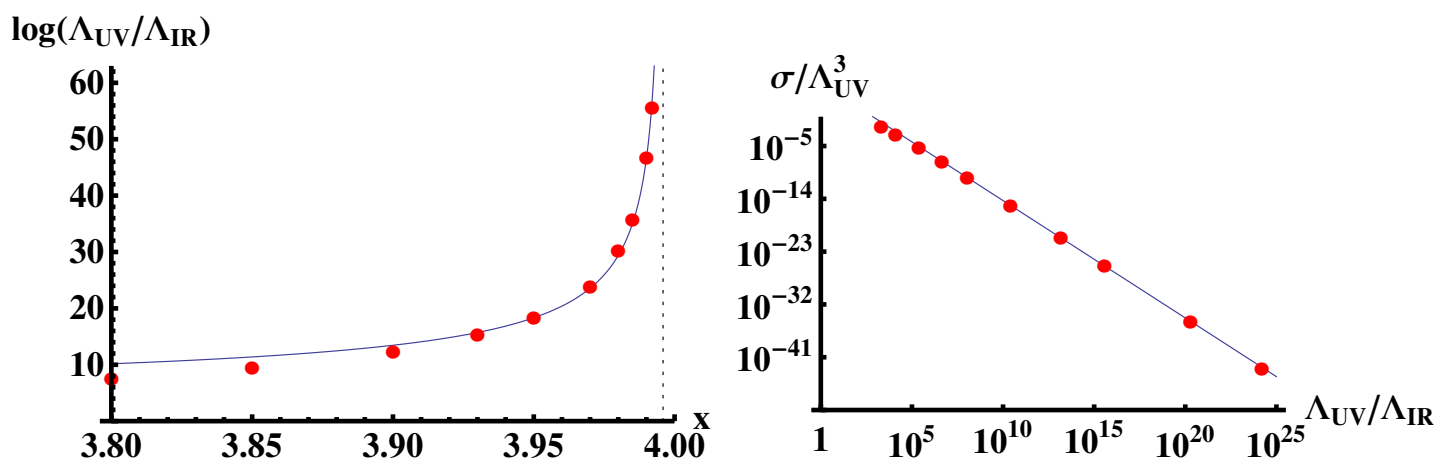

Figure 16. Left: $\log \left(\Lambda_{\mathrm{UV}} / \Lambda_{\mathrm{IR}}\right)$ as a function of $x$ (dots), compared to a BKT scaling fit (solid line). Right: $\sigma / \Lambda^{3}$ plotted against $\Lambda_{\mathrm{UV}} / \Lambda_{\mathrm{IR}}$ on log-log scale. The line is a fit with the expected power law, with power two, as shown in (10.27).

coupled to five-dimensional gravity background. Its characteristic feature is the holographic renormalization group flow of the YM coupling constant, identified as the exponential of the dilaton, as a function of the energy scale, identified roughly as the inverse of the bulk coordinate. On the other hand, the model builds on earlier work on including matter in holographic models via flavor branes in the quenched approximation, i.e., neglecting the backreaction of the brane on the dilaton and the background metric. In particular, we use the tachyon Dirac-Born-Infeld (DBI) action originally introduced by Sen.

Putting together these two frameworks, the dynamics in the Veneziano limit is modeled by a system of a dilaton and a tachyon coupled to five-dimensional gravity. The dilaton action is fixed as in IHQCD. For the tachyon we consider a generalized DBI action, where the dilaton dependence is parametrized in terms of a few potentials, which are a priori unknown. We may then constrain the unknown potentials, among other methods, by requiring that the UV physics implements that of (perturbative) QCD and that the solutions are regular in the IR.

The essential observation for uncovering the dynamics of the system, is the identification of the effective potential. It involves terms from both the dilaton and DBI actions, and takes the role of the dilaton potential of IHQCD. For large $x$, the perturbative Bank-Zaks IR fixed point can, and must be implemented trough the effective potential. Even further away from the Banks-Zaks region, the solutions can continue to flow to the fixed point in the IR. Interestingly, the fixed point can also be "screened" by the tachyon dynamics, such that the theory comes very close to it and the coupling almost freezes, but eventually starts running again in the deep IR. This kind of backgrounds are termed as the quasiconformal or "walking" ones.

In this article we did a detailed analysis of the backgrounds and the zero-temperature phase structure of V-QCD. Our main results are as follows:

- We generalize the holographic RG flow of IHQCD to include the evolutions of both the dilaton and the tachyon, which are controlled by the holographic $\beta$ - and $\gamma$-functions, respectively. Remarkably, for potentials that are analytic in the UV, the interplay of the dilaton and the tachyon automatically results in the anomalous 
dimension of the quark mass having physically reasonable UV asymptotics, i.e., a power series in the 't Hooft coupling.

- If we require that the UV expansions of the potentials capture the essential QCD physics, and choose potentials that join smoothly with their UV expansions, the VQCD phase diagram is always the physically relevant one. That is, for zero quark mass we find a phase transition at $x=x_{c}$. The conformal window, where the backgrounds have an IR fixed point, extends from $x=x_{c}$ up to the maximal value $x=11 / 2$ where asymptotic freedom is lost. Below $x=x_{c}$, chiral symmetry is broken, and the theory has similar behavior in the deep IR as the QCD we have observed in Nature.

- The edge of the conformal window is stabilized such that the dimension of the quark mass at the IR fixed point approaches two (the anomalous dimension approaches one) as the edge is approached. Under reasonable assumptions for the potentials, we find values of $x_{c}$ within a narrow band around the value $x_{c}=4$. High value of the anomalous dimension is of importance for applications to (walking) technicolor.

- Below $x_{c}$ but close to the edge of the conformal window, we find quasi-conformal or walking backgrounds.

- Backgrounds for (any) nonzero quark mass exist. In the conformal window the quark mass triggers chiral symmetry breaking, and below $x_{c}$ introducing the quark mass affects the scales of the theory in a physically reasonable manner.

- For $x>x_{c}$, the saddle point solution is always unique. At zero quark mass, the only regular tachyon solution is the trivial solution signaling unbroken chiral symmetry. At non-zero quark mass, the solution is still unique, but the tachyon is now non-trivial.

For $x<x_{c}$ there are $\infty+1$ "regular" saddle point solutions at zero quark mass. One of them is the trivial tachyon solution with unbroken chiral symmetry. The others are non-trivial tachyon solutions with a non-negative integer number of zeros realizing the Efimov minima. We call them Efimov saddle points. It is the solution with no zeros that is the lowest energy one, signaling chiral symmetry breaking.

At non-zero quark masses, there are still a finite number of saddle point solutions. The one with lowest energy is that with no zeros.

- Finally, as $x$ approaches $x_{c}$ from below for the backgrounds with zero quark mass, we show that the chiral condensate, as well as the length of energy range where the background stays close to the fixed point, obey the characteristic Miransky or BKT scaling law.

\section{Acknowledgments}

We thank D. Arean and C. Krishnan for contributions at an early stage of this project. We also thank D. D. Dietrich, U. Gürsoy, P. Hoyer, I. Iatrakis, N. Jokela, K. Kajantie, 
E. Keski-Vakkuri, D. Kutasov, M. Lippert, R. Meyer, T. Morita, V. Niarchos, A. Paredes, A. Parnachev and T. Ryttov for discussions and comments. This work was in part supported by the European grants FP7-REGPOT-2008-1: CreteHEPCosmo-228644 and PERG07-GA-2010-268246.

\section{A General solutions for $\beta$ and $\gamma$}

It is not difficult to construct numerically $\beta$ and $\gamma$ which solve the partial differential equations (7.5) and (7.6). We can use the fact that they were derived from a set of ordinary differential equations. More precisely, the equation have the form

$$
\begin{aligned}
& \beta \frac{\delta \beta}{\delta \lambda}+\gamma \frac{\delta \beta}{\delta T}=\mathcal{F}_{\beta}(\beta, \gamma) \\
& \beta \frac{\delta \gamma}{\delta \lambda}+\gamma \frac{\delta \gamma}{\delta T}=\mathcal{F}_{\gamma}(\beta, \gamma)
\end{aligned}
$$

where $\mathcal{F}_{\beta}$ and $\mathcal{F}_{\gamma}$ are independent of the partial derivatives of the $\beta$ - and $\gamma$-functions (and the dependence on potentials was left implicit).

Now we can apply a standard method for solving first order partial differential equations (PDEs), which in this case applies even for a pair of PDEs. We first look for curves along which the system reduces to ordinary DEs. Not surprisingly, such curves coincide with the holographic RG flow. That is, we can implicitly define such family of curves, by requiring that each curve $(\lambda(A), T(A))$, where $A$ parametrizes the curve, satisfies

$$
\lambda^{\prime}(A)=\beta(\lambda(A), T(A)) ; \quad T^{\prime}(A)=\gamma(\lambda(A), T(A)) .
$$

Then we notice that the differential operators in (A.1) and (A.2) become derivatives along the curves:

$$
\frac{d}{d A} \beta(\lambda(A), T(A))=\mathcal{F}_{\beta}(\beta(A), \gamma(A)) ; \quad \frac{d}{d A} \gamma(\lambda(A), T(A))=\mathcal{F}_{\gamma}(\beta(A), \gamma(A)) .
$$

Further, the equations along the curves are essentially in one-to-one correspondence with the system (5.8)-(5.11). Indeed, the original system of DEs can be formally recovered be eliminating the $\beta$ and $\gamma$ functions by using eqs. (A.3). Therefore, any solution of the original system satisfies the PDEs along the curve $(\lambda(r), T(r))$ that it defines.

As the final step of the method, we notice that the PDEs only depended on the derivatives of $\beta$ and $\gamma$ along the curves. The derivatives perpendicular to the curves can be freely chosen. Therefore, any (continuously parametrized) family of curves which satisfies (A.3) will define a solution to the PDEs in some region of the $(\lambda, T)$-plane. That is, the general solution to the PDEs is given by the planes that the solutions of eqs. (5.8)-(5.11) draw in $(\beta, \lambda, T)$ and $(\gamma, \lambda, T)$-spaces as the boundary conditions to the equations are varied in an arbitrary manner.

Remarkably, the amount of degrees of freedom of the solution matches with the expectation for a system of two first order PDEs, for which the solution should depend on two arbitrary functions. Since $\beta, \gamma, \lambda$, and $T$ are all invariant under the symmetry of (8.4), 
the number of integration constants in the system (5.8)-(5.11) which are relevant for the solution of PDEs is three.

A generic one-dimensional family of these parameters, and consequently a generic solution to the PDEs, can be defined by giving the dependence of two of these in terms of the third one which makes two arbitrary functions.

We have demonstrated how the boundary conditions of the PDEs are mapped to those of the original set of EoMs. Now the analysis of the solutions of the EoMs (see appendices D and E) suggests at least two natural ways to choose special one-parameter families of the boundary conditions, and to define special solutions to the PDEs.

1. Require that the solutions to EoMs end in the "good" IR singularity of section E.2.2 (with varying quark mass).

2. Require the more generic IR singularity of section E.2.1 and keep the quark mass fixed.

(In the second case, the "good" IR singularity is expected to arise as some limit of the more generic IR behavior so that it appears at a boundary of the region in $(\lambda, T)$-space where the $\beta$ and $\gamma$ functions are defined.)

\section{B A coordinate transformation}

It turns out that it is convenient to solve the system numerically using $A$ as a coordinate instead of $r$. Therefore, we present the system after this transformation:

$$
\begin{aligned}
12-\frac{6 \dot{q}}{q}+\frac{4 \dot{\lambda}^{2}}{3 \lambda^{2}} & =q^{2} V_{g}(\lambda)-q^{2} x V_{f}(\lambda, T) \sqrt{1+\frac{h(\lambda, T) \dot{T}^{2}}{q^{2}}} \\
12-\frac{4 \dot{\lambda}^{2}}{3 \lambda^{2}}= & q^{2} V_{g}(\lambda)-\frac{q^{2} x V_{f}(\lambda, T)}{\sqrt{1+q^{-2} h(\lambda, T) \dot{T}^{2}}} \\
\frac{\ddot{\lambda}}{\lambda}+\frac{4 \dot{\lambda}}{\lambda}-\frac{\dot{q}}{q} \frac{\dot{\lambda}}{\lambda}-\frac{\dot{\lambda}^{2}}{\lambda^{2}}= & \frac{3}{8} q^{2} \lambda\left(-\frac{d V_{g}}{d \lambda}+x \sqrt{1+q^{-2} h \dot{T}^{2}} \frac{\partial V_{g}}{\partial \lambda}+\frac{x \dot{T}^{2}}{2 q^{2} \sqrt{1+q^{-2} h \dot{T}^{2}}} \frac{\partial h}{\partial \lambda} V_{f}\right) \\
\ddot{T}+4 \dot{T}-\frac{\dot{q}}{q} \dot{T}= & -\frac{h \dot{T}^{3}}{q^{2}}\left[4+\dot{\lambda} \frac{\partial}{\partial \lambda} \log \left(\sqrt{h} V_{f}\right)\right]+\dot{T}^{2} \frac{\partial}{\partial T} \log \frac{V_{f}}{\sqrt{h}} \\
& -\dot{T} \dot{\lambda} \frac{\partial}{\partial \lambda} \log \left(h V_{f}\right)+\frac{q^{2}}{h} \frac{\partial}{\partial T} \log V_{f}
\end{aligned}
$$

where the dots are derivatives with respect to $A$ and we defined

$$
q(A)=e^{A} \frac{d r}{d A}
$$

\section{Examples of explicit potential choices}

We will construct explicit examples of potentials, which give physically reasonable backgrounds. We consider an Ansatz for $V_{f}$ of the form

$$
V_{f}(\lambda, T)=V_{f 0}(\lambda) e^{-a(\lambda) T^{2}}
$$


and assume that $h$ does not depend on $T$. First, we expect in the UV

$$
\begin{aligned}
V_{g}(\lambda) & =V_{0}+V_{1} \lambda+V_{2} \lambda^{2}+\cdots \\
V_{f}(\lambda, T=0) & =V_{f 0}(\lambda)=W_{0}+W_{1} \lambda+W_{2} \lambda^{2}+\cdots
\end{aligned}
$$

The potentials must also produce the good kind of IR singularity discussed in appendix E.2.2 with $P=1 / 2$ [71-73], which constrains the asymptotics of $V_{g}$ to

$$
V_{g}(\lambda) \sim \lambda^{4 / 3} \sqrt{\log \lambda} ; \quad \lambda \rightarrow \infty
$$

(assuming that $V_{f 0}$ plays no role in the IR for the chiral symmetry breaking solution as tachyon and/or $\lambda$ diverge so that $\exp \left(-a(\lambda) T^{2}\right)$ tends to zero).

In addition, the IR behavior of $V_{f 0}$ should be chosen such that the fixed point (maximum) of $V_{g}(\lambda)-x V_{f 0}(\lambda)$, which is guaranteed to be present at large $x \rightarrow 11 / 2$ if we fix the small $\lambda$ series of the potential appropriately, continues to exists up to sufficiently low $x$. This is most easily achieved if $V_{f 0}$ diverges faster than $V_{g}$ as $\lambda \rightarrow \infty$, so that the fixed point exists for all $x$. Another possibility, mentioned in section 6 , is that the fixed point exist only for $x \geq x_{*}$, where $x_{*}>0$ is relatively small. We have checked that choosing such potentials does not change any results at qualitative level, and do not discuss this choice further here. We also require that the potentials are analytic at $\lambda=0$. A simple Ansatz that meets these requirements and involves free coefficients up to two-loop order is

$$
\begin{aligned}
V_{g}(\lambda) & =V_{0}+V_{1} \lambda+V_{2} \frac{\lambda^{2}}{\left(1+\lambda / \lambda_{0}\right)^{2 / 3}} \sqrt{1+\log \left(1+\lambda / \lambda_{0}\right)} \\
V_{f 0}(\lambda) & =W_{0}+W_{1} \lambda+W_{2} \lambda^{2} .
\end{aligned}
$$

Notice that the AdS radius

$$
\ell=\sqrt{\frac{12}{V_{0}-x W_{0}}}
$$

must be well defined for all $x$ up to $x=11 / 2$, which sets an upper bound for $W_{0}$ for given $V_{0}$. We also expect $V_{f}$ to be positive at small $\lambda,{ }^{28}$ as can be seen from the probe calculation and analyzed in [67].

Therefore, we take

$$
0 \leq W_{0} \leq \frac{2}{11} V_{0}
$$

However, as discussed in appendix D.1.1, the sum of the anomalous dimensions of the quark mass and the chiral condensate is not equal to 4 if $W_{0}=0$ (then $\delta=1$ in appendix D.1.1). Therefore, we discard this option. Notice also that if we saturate the upper limit with $W_{0}=\frac{2}{11} V_{0}$, the AdS radius diverges in the Banks-Zaks limit $x \rightarrow 11 / 2$ unless we choose an $x$-dependent $V_{0}$. Notice also that (C.8) assumed a constant $W_{0}$. In the generic case of $x$-dependent $W_{0}$ we need to require instead that $0<W_{0}<V_{0} / x$ for all values of $x$ within the range $0<x<11 / 2$.

\footnotetext{
${ }^{28}$ Negative $W_{0}$ is also problematic since, at least for small $\left|W_{0}\right|$, it generates a zero of $V_{f}$, which causes the tachyon solution to become singular.
} 
In addition, we can fix the coefficients $V_{i}, W_{i}$ by mapping to the field theory $\beta$-functions as

$$
\begin{aligned}
V_{g}(\lambda) & =\frac{12}{\ell_{0}^{2}}\left[1+\frac{8}{9} b_{0}^{\mathrm{YM}} \lambda+\frac{23\left(b_{0}^{\mathrm{YM}}\right)^{2}-36 b_{1}^{\mathrm{YM}}}{81} \lambda^{2}+\cdots\right] \\
V_{g}(\lambda)-x V_{f 0}(\lambda) & =\frac{12}{\ell^{2}}\left[1+\frac{8}{9} b_{0} \lambda+\frac{23 b_{0}^{2}-36 b_{1}}{81} \lambda^{2}+\cdots\right] .
\end{aligned}
$$

Here the (scheme independent) QCD $\beta$-function in the Veneziano limit with vanishing quark masses up to two-loop order are

$$
b_{0}=\frac{2}{3} \frac{11-2 x}{(4 \pi)^{2}} ; \quad b_{1}=-\frac{2}{3} \frac{34-13 x}{(4 \pi)^{4}}
$$

and $b_{i}^{\mathrm{YM}}=\left.b_{i}\right|_{x=0}$. Setting $V_{0}=12$ and $W_{0}=12 / 11$, which lies in the middle of the allowed region of eq. (C.8), we obtain

$$
\begin{aligned}
V_{1} & =\frac{44}{9 \pi^{2}} & V_{2} & =\frac{4619}{3888 \pi^{4}} ; \\
W_{1} & =\frac{4(33-2 x)}{99 \pi^{2}} & W_{2} & =\frac{23473-2726 x+92 x^{2}}{42768 \pi^{4}} .
\end{aligned}
$$

Further, we choose $\lambda_{0}=8 \pi^{2}$ to prevent the higher order terms in the UV expansion of the potentials from growing unnaturally large.

In addition, we need to choose the functions $a(\lambda)$ and $h(\lambda)$ appropriately. As discussed in section 8 and in appendix D.1.1, we must have

$$
\frac{h(\lambda)}{a(\lambda)}=\frac{2 \ell^{2}}{3}\left(1+h_{1} \lambda+\cdots\right)
$$

Here the coefficient $h_{1}$ can be matched with the one-loop anomalous dimension of the quark mass, which reads in the Veneziano limit

$$
\gamma_{m}(\lambda)=\frac{3}{(4 \pi)^{2}} \lambda+\cdots
$$

By matching with eq. (D.10) from appendix, we obtain

$$
-\frac{3}{(4 \pi)^{2}}=\frac{9}{8}\left[\frac{4}{3} \frac{8}{9} b_{0}+\frac{4}{3} h_{1}\right]
$$

from which

$$
h_{1}=-\frac{115-16 x}{216 \pi^{2}} .
$$

The IR behavior of the functions $h(\lambda)$ and $a(\lambda)$ is linked to the tachyon behavior in the IR for the solution which breaks chiral symmetry. They are discussed in detail in appendix E.2.2, where essentially only two different cases, which are consistent with the good IR singularity, are found. These possibilities are produced by the following choices. 
I We can choose the function $a(\lambda)$ to be constant, and the function $h(\lambda)$ to have powerlaw IR asymptotics:

$$
h(\lambda)=\frac{1}{\left(1-\frac{3 h_{1}}{4} \lambda\right)^{4 / 3}} ; \quad a(\lambda)=a_{0}=\frac{3}{2 \ell^{2}}=\frac{3}{22}(11-x),
$$

which corresponds to the special case of $\rho=4 / 3$ and $\sigma=0$ in appendix E.2.2. In this case the tachyon diverges exponentially,

$$
T(r) \sim T_{0} \exp \left[\frac{813^{5 / 6}(115-16 x)^{4 / 3}(11-x)}{8129442^{1 / 6}} \frac{r}{R}\right]
$$

as $r \rightarrow \infty$. Here $R$ is the IR scale of the solutions which is defined by eqs. (E.18), (E.20) and (E.21). $T_{0}$ is the only free parameter.

II The other choice is practically a generalization of the first one. It has a simple form of $h(\lambda)$, but more complicated $a(\lambda)$ :

$$
h(\lambda)=\frac{1}{\left(1+\lambda / \lambda_{0}\right)^{4 / 3}} ; \quad a(\lambda)=\frac{3}{22}(11-x) \frac{1-h_{1} \lambda+h_{2} \lambda^{2}}{\left(1+\lambda / \lambda_{0}\right)^{4 / 3}},
$$

so that $\rho=4 / 3$ and $\sigma=2 / 3$ in appendix E.2.2. The extra term proportional to $h_{2}$ was added to make $\sigma$ positive. We choose its coefficient to be small, $h_{2}=1 / \lambda_{0}^{2}$. The tachyon behaves as

$$
T(r) \sim \frac{272^{3 / 4} 3^{1 / 4}}{\sqrt{4619}} \sqrt{\frac{r-r_{1}}{R}}
$$

for large $r$. Here $r_{1}$ is a free parameter.

We have checked that both scenarios lead to qualitatively similar results. In the numerical calculations we use, for definiteness, the choice I, unless stated otherwise.

\section{UV behavior}

In this appendix we shall discuss the UV behavior of the system (5.8)-(5.11) in general. This analysis should be compared to that carried out in $[71-73,76]$ in the absence of the tachyon backreaction. Recall that apart from the two degrees of freedom of the transformation (8.4), the solutions contain three integration constants. In the discussion below, the degrees of freedom refer to these three "nontrivial" constants.

We shall not discuss the most general behavior of the solutions, but make some physically motivated assumptions. In particular, we restrict ourselves to the potentials $V_{g}$ and $V_{f}$ which are bounded as $\lambda \rightarrow 0$, and which are smooth at any finite $\lambda$. In general we are interested two types of potentials: ones that start from a constant value at $\lambda=0$, are monotonic as $\lambda$ increases, and approach $+\infty$ as $\lambda \rightarrow \infty$, and ones that start at a constant value at $\lambda=0$, increase until they reach a maximum at some $\lambda=\lambda_{*}$, and thereafter monotonically decrease to $-\infty$ as $\lambda \rightarrow \infty$. This should be kept in mind while reading the analysis below, as some of the arguments below may fail for more generic potentials, even though no assumptions are listed explicitly. Further, we mostly restrict to effective $\beta$-functions $\beta_{\mathrm{eff}}=d \lambda / d A$ which are negative. 


\section{D.1 Generic behavior}

\section{D.1.1 The UV AdS boundary at $\lambda=0$}

Near the standard UV singularity, the tachyon must behave schematically as $T(r) \sim m r+$ $\sigma r^{3}$ whereas the other fields have a logarithmic dependence on $r$. Therefore the tachyon decouples asymptotically as $r \rightarrow 0$, and we may analyze its UV behavior by first solving $A$ and $\lambda$ with $T=0$ and then by analyzing the tachyon EoM for this background.

Asymptotic behavior of $\boldsymbol{A}$ and $\lambda$. We take

$$
V_{\mathrm{eff}}(\lambda)=V_{g}(\lambda)-x V_{f}(\lambda, 0)=\frac{12}{\ell^{2}}\left[1+V_{1} \lambda+V_{2} \lambda^{2}+\cdots\right] .
$$

Then the (leading) UV expansions of $A$ and $\lambda$ can be written as

$$
\begin{aligned}
A(r)= & -\log \frac{r}{\ell}+\frac{4}{9 \log (r \Lambda)} \\
& +\frac{\frac{1}{162}\left[95-\frac{64 V_{2}}{V_{1}^{2}}\right]+\frac{1}{81} \log [-\log (r \Lambda)]\left[-23+\frac{64 V_{2}}{V_{1}^{2}}\right]}{\log (r \Lambda)^{2}}+\mathcal{O}\left(\frac{1}{\log (r \Lambda)^{3}}\right) \\
V_{1} \lambda(r)= & -\frac{8}{9 \log (r \Lambda)}+\frac{\log [-\log (r \Lambda)]\left[\frac{46}{81}-\frac{128 V_{2}}{81 V_{1}^{2}}\right]}{\log [r \Lambda]^{2}}+\mathcal{O}\left(\frac{1}{\log (r \Lambda)^{3}}\right) .
\end{aligned}
$$

Notice that they contain no free parameters (in addition to $\Lambda$ ). In fact, after using the equations of motion there is one degree of freedom left in the coefficients of the above expansion, but as it turns out, this freedom can be eliminated by rescaling $\Lambda$. We have removed this extra parameter by requiring that the coefficient of the $1 /(\log r \Lambda)^{2}$ term in the expansion of $\lambda$ vanishes.

Tachyon UV asymptotics. We take

$$
V_{f}(\lambda, T)=e^{-a(\lambda) T^{2}} V_{f}(\lambda)
$$

and parametrize

$$
\begin{aligned}
V_{\mathrm{eff}}(\lambda) & =V_{g}(\lambda)-x V_{f}(\lambda, 0)=\frac{12}{\ell^{2}}\left[1+V_{1} \lambda+V_{2} \lambda^{2}+\cdots\right] \\
x V_{f}(\lambda) & =\lambda^{\delta}\left[W_{0}+W_{1} \lambda+W_{2} \lambda^{2}+\cdots\right] \\
\frac{h(\lambda)}{a(\lambda)} & =\frac{2 \ell^{2}}{3}\left[1+h_{1} \lambda+h_{2} \lambda^{2}+\cdots\right]
\end{aligned}
$$

where $\delta$ is a nonnegative integer. Here the leading coefficient of $h / a$ was already fixed in order to have the correct UV mass of the tachyon [91]. We further assume that

$$
h(\lambda)=\lambda^{\xi}(1+\mathcal{O}(\lambda)) .
$$

It is enough to study the linear terms in the tachyon EoM, which read

$$
\begin{aligned}
T^{\prime \prime}(r) & +\left[-3-\frac{\delta+\xi}{\log (r \Lambda)}+\mathcal{O}\left(\frac{1}{\log (r \Lambda)^{2}}\right)\right] \frac{T^{\prime}(r)}{r} \\
& +\left[3+\frac{8\left(h_{1}+V_{1}\right)}{3 V_{1} \log (r \Lambda)}+\mathcal{O}\left(\frac{1}{\log (r \Lambda)^{2}}\right)\right] \frac{T(r)}{r^{2}}=0 .
\end{aligned}
$$


From this, we would naively expect a solution of the form

$$
T(r) \sim m r(1+\mathcal{O}(1 / \log r))+\sigma r^{3}(1+\mathcal{O}(1 / \log r))
$$

i.e., the logarithmically suppressed corrections to the EoM show up as logarithmically suppressed corrections to the functions. However, this is not the case: an Ansatz for the solution which assumes this kind of corrections fails. The correct asymptotics reads

$$
\begin{aligned}
\frac{1}{\ell} T(r)= & m r(-\log (r \Lambda))^{\frac{4}{3}-\frac{\delta}{2}-\frac{\xi}{2}+\frac{4 h_{1}}{3 V_{1}}}\left[1+\frac{C_{1}+C_{2} \log (-\log (r \Lambda))}{\log (r \Lambda)}+\mathcal{O}\left(\frac{1}{\log (r \Lambda)^{2}}\right)\right](\mathrm{D} .9) \\
& +\sigma r^{3}(-\log (r \Lambda))^{-\frac{4}{3}+\frac{3 \delta}{2}+\frac{3 \xi}{2}-\frac{4 h_{1}}{3 V_{1}}}\left[1+\frac{D_{1}+D_{2} \log (-\log (r \Lambda))}{\log (r \Lambda)}+\mathcal{O}\left(\frac{1}{\log (r \Lambda)^{2}}\right)\right]
\end{aligned}
$$

where $C_{i}$ and $D_{i}$ are computable functions of $\delta, \xi, h_{1}, V_{1}, V_{2}, W_{1}$, and $W_{2}$.

The "surprising" logarithmic power corrections in eq. (D.9) can actually be identified as the nontrivial running of the quark mass and the condensate in the UV, which arises as their anomalous dimensions are different from zero. To make this explicit, we calculate the gamma function $T^{\prime} / A^{\prime}$ in the UV. For $m \neq 0$ it is dominated by the linear tachyon solution:

$$
\frac{\gamma}{T}=\frac{T^{\prime}}{T A^{\prime}}=-1-\frac{\frac{4}{3}-\frac{\delta+\xi}{2}+\frac{4 h_{1}}{3 V_{1}}}{\log (r \Lambda)}+\mathcal{O}\left(\frac{1}{\log (r \Lambda)^{2}}\right)
$$

whereas for $m=0$ we find

$$
\frac{\gamma}{T}=-3+\frac{\frac{4}{3}-\frac{3(\delta+\xi)}{2}+\frac{4 h_{1}}{3 V_{1}}}{\log (r \Lambda)}+\mathcal{O}\left(\frac{1}{\log (r \Lambda)^{2}}\right) .
$$

The next-to-leading terms in (D.10) and (D.11) are mapped to the one-loop anomalous dimensions of the quark mass and the chiral condensate in QCD, respectively. Since they should add up to zero, we must have $\delta+\xi=0$. The easiest way to satisfy this is to take $\delta=0=\xi$. In particular, the expression $h(\lambda)=\lambda^{-4 / 3}$ with $\xi=-4 / 3$, which was found in the probe limit [71-73], does not work, since $\delta$ was required to be an integer to ensure that the $\beta$-functions have power series with integer powers at $\lambda=0$.

Finally, it is easy to verify that the UV expansion presented here match with those of section 7.1 , which were derived by using the holographic beta functions.

\section{D.1.2 A bounce-back at finite $\lambda$}

A bounce-back may take place when the potential $V_{\text {eff }}(\lambda)=V_{g}(\lambda)-x V_{f}(\lambda, T=0)$ has a maximum at some $\lambda=\lambda_{*}$ signaling the presence of an fixed point, and $V^{\prime}(\lambda)<0$ for $\lambda>\lambda_{*}$. If the tachyon is sufficiently small, the effective $\beta$-function $d \lambda / d A$ hits zero, and becomes positive when the system is evolved towards the UV. Therefore the coupling has a finite minimum $\left(>\lambda_{*}\right)$ and the above "standard" UV asymptotics at $\lambda \rightarrow 0$ is not reached. All fields are analytic at the point where $\lambda^{\prime}=0$. The bounce-back behavior is found in the white regions of figure 5. Examples of the $\beta$ and $\gamma$-functions evaluated along the RG flow for the bounce-back scenario are shown as the dotted curves in figure 17 in appendix F. 


\section{D.2 Special case: UV fixed point at finite $\lambda$}

We have identified one special UV asymptotics, which is found as a limiting case between the two generic behaviors discussed above (the blue curve of figure 5). In this case the asymptotic solution is expected to depend on two integration constants. The solution terminates as the $\beta$-function $d \lambda / d A$ approaches zero at the maximum $\lambda_{*}$ of the effective potential $V_{\text {eff }}(\lambda)=V_{g}(\lambda)-x V_{f}(\lambda, T=0)$. This happens at fixed value of $r=r_{*}$ where $A$ also diverges and $\lambda$ approaches $\lambda_{*}$ from above. Examples of the $\beta$ and $\gamma$-functions evaluated along the RG flow with this UV fixed point are shown as the thick blue curves of the middle and right columns of figure 17 in appendix F.

Depending on the value of the tachyon mass at the fixed point (see section 8.3), the asymptotics may be written in two different forms. Let us recall the definition of the dimension $\Delta$ at the fixed point:

$$
\Delta(4-\Delta)=\frac{24 a\left(\lambda_{*}\right)}{h\left(\lambda_{*}\right) V_{\mathrm{eff}}\left(\lambda_{*}\right)} .
$$

When $x>x_{c}$ the right hand side of the definition is smaller than 4 so that there are two real roots $\Delta=\Delta_{ \pm}$. The geometry approaches the AdS one near the fixed point (which we place at the origin, $r_{*}=0$, by using translation symmetry),

$$
\begin{aligned}
A(r) & =-\log r+\log \ell_{*}+A_{0}(\Lambda r)^{2 \Delta_{-}}+\cdots \\
\lambda(r) & =\lambda_{*}+\lambda_{0}(\Lambda r)^{2 \Delta_{-}}+\cdots \\
T(r) & =T_{0}(\Lambda r)^{\Delta_{-}}+\cdots
\end{aligned}
$$

where $\Delta_{-}$is the smaller root, $\ell_{*}^{2}=12 / V_{\text {eff }}\left(\lambda_{*}\right)$, and the constants $A_{0}, \lambda_{0}$, and $T_{0}$ satisfy two constraints which can be solved from the EoMs (5.8)-(5.11). There are two free parameters which can be taken to be the coefficients of the tachyon solutions with the dimensions $\Delta_{ \pm}$. The solution associated to $\Delta_{+}$will appear at the next-to-leading order only if we choose the coefficient $T_{0}$ of the above solution to vanish.

When $x<x_{c}$ we have two complex roots $\Delta_{ \pm} \equiv 2 \pm i k$. Now the asymptotics reads

$$
\begin{aligned}
A(r) & =-\log r+\log \ell_{*}+(\Lambda r)^{4}\left[A_{1}+\hat{A}_{1} \sin \left(2 k \log (\Lambda r)+\phi_{A}\right)\right]+\cdots \\
\lambda(r) & =\lambda_{*}+(\Lambda r)^{4}\left[\lambda_{1}+\hat{\lambda}_{1} \sin \left(2 k \log (\Lambda r)+\phi_{\lambda}\right)\right]+\cdots \\
T(r) & =T_{0}(\Lambda r)^{2} \sin \left(k \log (\Lambda r)+\phi_{T}\right)+\cdots
\end{aligned}
$$

The coefficients in the next-to-leading terms for $\lambda$ and $A$ can be solved from the EoMs (5.8)-(5.11) by inserting the tachyon asymptotics. The free parameters are $T_{0}$ and $\phi_{T}$ in this case.

\section{E IR behavior}

In this appendix we discuss the IR behavior of the system (5.8)-(5.11) in general. As above, we restrict to simple potentials and to cases where the $\beta$-function $d \lambda / d A$ takes negative 
values in the vicinity of the IR singularity. The IR structure is much richer than the UV one, mostly because there are much less obvious constraints on the potentials.

We shall discuss here both generic and special IR asymptotics. Of these, the most interesting ones for us will be the the special ones that depend only on one free parameter (excluding trivial reparametrization symmetries). They can be identified as the "good" or acceptable or repulsive, IR singularities, [71-73, 76, 121]. Examples of such singularities are identified below in section E.2.3 partially based on the analysis of section E.2.2.

\section{E.1 Generic cases}

There are two generic IR asymptotics which in fact do not involve divergences of any of the fields. Therefore, they also appear independently of the details of the potentials to a large extent. They are not acceptable asymptotics.

\section{E.1.1 Divergence of the derivative of the tachyon}

A typical, generic IR behavior is similar to what was found in the probe limit in [71-73], where the tachyon is regular but its derivative diverges. Indeed, the Ansatz

$$
\begin{aligned}
T(r) & =T_{*}+T_{1} \sqrt{r_{*}-r}+T_{2}\left(r_{*}-r\right)+\cdots \\
A(r) & =A_{*}+A_{1}\left(r_{*}-r\right)+A_{2}\left(r_{*}-r\right)^{3 / 2}+\cdots \\
\lambda(r) & =\lambda_{*}+\lambda_{1}\left(r_{*}-r\right)+\lambda_{2}\left(r_{*}-r\right)^{3 / 2}+\cdots
\end{aligned}
$$

solves the equations of motion (5.8)-(5.11) in general. We do not present the complicated constraint equations which follow for the constants in the expansions, but it is not difficult to check that the solution has three independent integration constants and is therefore generic. Since none of the fields diverges at $r=r_{*}$, it is natural to take all the potentials to be analytic at the point of expansion, and the solution is expected to exist to a large extent independently of the choices for them.

Notice also that there is no real singularity at $r=r_{*}$ : one can make a coordinate transformation such that all fields are analytic in the vicinity of this point. A natural choice that realizes this is to use $T$ as the coordinate. Of course, this leads to all fields being double-valued functions of $r$, with the two branches having the same absolute value of $T_{1}$ but opposite signs. If this is allowed, it is not hard to find analytic solutions which, for example, start at a UV singularity at $\lambda=0$, bounce back at a point where the tachyon derivative diverges, and return to another singularity at $\lambda=0$.

\section{E.1.2 A bounce-back as $d \lambda / d A \rightarrow 0$}

There is also a three-dimensional space of solutions where the coupling reaches a maximum value, and then starts to decrease with decreasing $A$ so that the $\beta$-function $d \lambda / d A$ becomes positive. All fields are analytic in $r$ at the point where the $\beta$-function is zero. We have not checked how the solutions continue to evolve in the region of positive $\beta$-function. 


\section{E.2 Special IR singularities}

In addition to the generic IR behaviors discussed above, we have identified several special IR singularities, where the fields $A$ and $\lambda$ diverge. Note that above, the divergence of the tachyon derivative was proportional to the parameter $T_{1}$, which could take both positive and negative values. Solutions with arbitrary small $\left|T_{1}\right|$ also exist, as well as the limit $T_{1} \rightarrow 0$ where the solution, which in general ends in the divergence of $A$ and $\lambda$ rather than $T$. Therefore we expect that spaces of generic solutions with positive and negative $T_{1}$, respectively, will be separated by a subspace of solutions with singularity in the IR. The behavior of the system in this case depends strongly on the asymptotics of the potentials. However, the tachyon often decouples asymptotically from $\lambda$ and $A$, in particular if the tachyon diverges in the IR, which is the expected behavior for physically relevant singularities $[91,101,102]$. Here we shall assume that the decoupling takes place, since completely general classification of the singularities seems daunting.

As the tachyon decouples, the classification of singularities for $A$ and $\lambda$ follows earlier studies $[71-73,76]$. We shall review the results here for clarity. Assuming that the tachyon tends to $T_{0}$ in the IR, the effective potential that drives the metric and the coupling in the IR is

$$
V_{\mathrm{IR}}(\lambda)=V_{g}(\lambda)-x V_{f}\left(\lambda, T_{0}\right)
$$

where $T_{0}$ can be infinite in which case $V_{\mathrm{IR}}(\lambda)=V_{g}(\lambda)$. We parametrize

$$
V_{\mathrm{IR}}(\lambda)=\lambda^{2 Q}(\log \lambda)^{P}\left(V_{0}+\frac{V_{1}}{\log \lambda}+\frac{V_{2}}{(\log \lambda)^{2}}+\cdots\right)
$$

as $\lambda \rightarrow \infty$. The equations of motion are eqs. (5.8) and (5.9) with $T(r) \equiv T_{0}$ so that $T^{\prime}(r)=0$. There are two types of singularities (see [76]): "generic" ones where

$$
X=\frac{1}{3 \lambda} \beta=\frac{1}{3 \lambda} \frac{d \lambda}{d A} \rightarrow-1
$$

in the IR, and "special" ones where $X \rightarrow-\frac{3}{4} Q$.

\section{E.2.1 Generic metric singularity}

The generic singularities exist for $Q \leq 4 / 3$ and depend on one free parameter. The system is solved by the Ansatz

$$
\begin{aligned}
A & =\frac{1}{3} \log \delta r-\log R+A_{1} \delta r^{(8-6 Q) / 3}(-\log \delta r)^{P}+\cdots \\
\lambda & =\frac{1}{\delta r}\left(\lambda_{0}+\lambda_{1} \delta r^{(8-6 Q) / 3}(-\log \delta r)^{P}+\cdots\right)
\end{aligned}
$$

where

$$
\delta r=\left(r_{*}-r\right) / R
$$

is the "conformally invariant" distance from the singularity, $\lambda_{0}$ is the free parameter, and the dropped terms are suppressed by $1 / \log \delta r$. Plugging this in the equations of motion,

$$
\begin{aligned}
A_{1} & =\frac{3 V_{0} \lambda_{0}^{2 Q}}{88-114 Q+36 Q^{2}} \\
\lambda_{1} & =-\frac{27(2 Q-1) V_{0} \lambda_{0}^{2 Q+1}}{16\left(44-57 Q+18 Q^{2}\right)} .
\end{aligned}
$$


For $Q=4 / 3$ the singularity exists if $P<1$. In this case the asymptotics reads

$$
\begin{aligned}
A & =\frac{1}{3} \log \delta r-\log R+\frac{V_{0} \lambda_{0}^{8 / 3}}{3(1+P)}(-\log \delta r)^{P+1}+\cdots \\
\lambda & =\frac{1}{\delta r}\left(\lambda_{0}-\frac{5 V_{0} \lambda_{0}^{11 / 3}}{8(1+P)}(-\log \delta r)^{P+1}+\cdots\right) .
\end{aligned}
$$

\section{E.2.2 Special metric singularity}

This kind of singularity exist for $0<Q<4 / 3$ so that the asymptotic value $-4 Q / 3$ of $X$ lies between zero and one. We need to require that the potential is asymptotically positive, $V_{0}>0$. The solution does not involve any integration constants in addition to the ones linked to the reparametrization symmetry, which suggest that when combined with a proper tachyon solution, acceptable IR asymptotics can be identified.

If $2 / 3<Q<4 / 3$ we find a singularity at finite value $r_{*}$ of $r$ :

$$
\begin{aligned}
A= & \frac{1}{9 Q^{2} / 4-1} \log \delta r-\log R+\mathcal{O}(1 / \log \delta r) \\
\lambda= & -\frac{Q}{Q^{2}-4 / 9} \log \delta r+\frac{1}{2 Q}\left[2 \log 2+(1-2 P) \log 3+(P-2) \log \left(9 Q^{2}-4\right)\right. \\
& \left.+\log \left(16-9 Q^{2}\right)-P \log Q-P \log (-\log \delta r)-\log V_{0}\right]+\mathcal{O}(1 / \log \delta r)
\end{aligned}
$$

where again $\delta r=\left(r_{*}-r\right) / R$. Here $R$ and $r_{*}$ are the integration constants which reflect the reparametrization symmetry, but no other free parameters appear.

If $0<Q<2 / 3$ similar formulas hold for $r \rightarrow \infty$ :

$$
\begin{aligned}
A= & -\frac{1}{1-9 Q^{2} / 4} \log \hat{r}-\log R+\mathcal{O}(1 / \log \hat{r}) \\
\lambda= & \frac{Q}{4 / 9-Q^{2}} \log \hat{r}+\frac{1}{2 Q}\left[2 \log 2+(1-2 P) \log 3+(P-2) \log \left(4-9 Q^{2}\right)\right. \\
& \left.+\log \left(16-9 Q^{2}\right)-P \log Q-P \log (\log \hat{r})-\log V_{0}\right]+\mathcal{O}(1 / \log \hat{r})
\end{aligned}
$$

where now $\hat{r}=\left(r-r_{0}\right) / R$.

If $Q=2 / 3$, and $P<1$, there is a singularity at $r=\infty$. The asymptotic solution reads

$$
\begin{aligned}
A= & -\left(\frac{r-r_{0}}{R}\right)^{\alpha}+A_{0}-\frac{1}{2} \frac{P}{1-P} \log \frac{R}{r-r_{0}}+\frac{5}{6}+\frac{P}{4}+\frac{1}{2} P \log \frac{3}{2}+\frac{2 V_{1}}{3 P V_{0}} \\
& +\frac{-52 P^{2} V_{0}^{2}+4 P^{3} V_{0}^{2}+27 P^{4} V_{0}^{2}+64 V_{1}^{2}-64 P V_{1}^{2}+128 P V_{0} V_{2}}{288 P(1+P) V_{0}^{2}}\left(\frac{R}{r-r_{0}}\right)^{\alpha}+\cdots \\
\log \lambda= & +\frac{3}{2}\left(\frac{r-r_{0}}{R}\right)^{\alpha}-\frac{5}{4}-\frac{3 P}{8}-\frac{V_{1}}{P V_{0}} \\
& +\frac{-20 P^{2} V_{0}^{2}-40 P^{3} V_{0}^{2}+9 P^{4} V_{0}^{2}-64 V_{1}^{2}+64 P V_{1}^{2}-128 P V_{0} V_{2}}{192 P(1+P) V_{0}^{2}}\left(\frac{R}{r-r_{0}}\right)^{\alpha}+\cdots
\end{aligned}
$$

where

$$
\begin{aligned}
\alpha & =\frac{1}{1-P} \\
R & =\frac{2^{P} 3^{1-P}}{(1-P) e^{A_{0}} \sqrt{V_{0}}}
\end{aligned}
$$


As pointed out in [71-73], this special case produces a good match with the IR physics of QCD, in particular if we choose $P=1 / 2$. We will confirm below that the potentials of the tachyon action can be chosen such that the tachyon asymptotics also meets all requirements known to us, and the produced singularity is of the "good" kind. We will use these singularities in our analysis, and the above formulas will be used to fix the IR boundary conditions for the numerical solutions.

If $Q=2 / 3$, and $P>1$, we find a singularity at finite value $r=r_{*}$ of the coordinate. The asymptotics transforms to

$$
\begin{aligned}
A= & -\left(\frac{R}{r_{*}-r}\right)^{\bar{\alpha}}+A_{0}-\frac{1}{2} \frac{P}{1-P} \log \frac{R}{r_{*}-r}+\frac{5}{6}+\frac{P}{4}+\frac{1}{2} P \log \frac{3}{2}+\frac{2 V_{1}}{3 P V_{0}} \\
& +\frac{-52 P^{2} V_{0}^{2}+4 P^{3} V_{0}^{2}+27 P^{4} V_{0}^{2}+64 V_{1}^{2}-64 P V_{1}^{2}+128 P V_{0} V_{2}}{288 P(1+P) V_{0}^{2}}\left(\frac{r_{*}-r}{R}\right)^{\bar{\alpha}}+\cdots \\
\log \lambda= & +\frac{3}{2}\left(\frac{R}{r_{*}-r}\right)^{\bar{\alpha}}-\frac{5}{4}-\frac{3 P}{8}-\frac{V_{1}}{P V_{0}} \\
& +\frac{-20 P^{2} V_{0}^{2}-40 P^{3} V_{0}^{2}+9 P^{4} V_{0}^{2}-64 V_{1}^{2}+64 P V_{1}^{2}-128 P V_{0} V_{2}}{192 P(1+P) V_{0}^{2}}\left(\frac{r_{*}-r}{R}\right)^{\bar{\alpha}}+\cdots
\end{aligned}
$$

where $\bar{\alpha}=1 /(P-1)$ and $A_{0}$ is related to $R$ as in eq. (E.21).

Finally, for $Q=2 / 3$ and $P=1$ the metric factor $A$ diverges exponentially as $r \rightarrow \infty$,

$$
\begin{aligned}
A= & -\exp \left(\frac{r-r_{0}}{R}\right)-\log R+\frac{r-r_{0}}{2 R}+\frac{1}{2} \log 6-\frac{1}{2} \log V_{0}+\frac{13}{12}+\frac{2 V_{1}}{3 V_{0}} \\
& +\frac{128 V_{2}-21 V_{0}}{576 V_{0}} \exp \left(-\frac{r-r_{0}}{R}\right)+\cdots \\
\log \lambda= & +\frac{3}{2} \exp \left(\frac{r-r_{0}}{R}\right)-\frac{V_{1}}{V_{0}}-\frac{13}{8}+\frac{-51 V_{0}-128 V_{2}}{384 V_{0}} \exp \left(-\frac{r-r_{0}}{R}\right)+\cdots .
\end{aligned}
$$

\section{E.2.3 Tachyon behavior}

To complete the analysis, one should insert each of the above asymptotics to the tachyon EoM and check what the tachyon asymptotics is for various choices of the potentials $V_{f}$ and $h$, and start looking for the "good" kind of singularities. Once the potentials are fixed, one can check if a solution, which is consistent with the assumption that the tachyon decouples, indeed exists. If it does, it can depend on one or two additional parameters. For the good, fully repulsive singularities the number of free parameters (excluding those related to the reparametrization symmetry) is equal to one, i.e., we must have a special metric singularity combined with a one-parameter tachyon asymptotics. In addition we should require that the tachyon diverges in the IR, since that kind of solutions have bulk flavor anomalies similar to those of QCD [91, 101, 102].

Here we shall restrict to the special metric IR singularity with $Q=2 / 3$ and $P<1$, since it is expected to include the most interesting cases due to additional constraints from confinement and excitation spectra [71-73]. We parametrize

$$
V_{f}(\lambda, T)=V_{f 0}(\lambda) \exp \left(-a(\lambda) T^{2}\right) ; \quad h=h(\lambda) .
$$


With this parametrization, the tachyon EoM reads

$$
T^{\prime \prime}+F_{1} T^{\prime}+F_{2} T+F_{3} T^{\prime 3}+F_{4} T^{\prime 2} T+F_{5} T^{\prime} T^{2}+F_{6} T^{\prime 3} T^{2}=0
$$

where

$$
\begin{array}{llrl}
F_{1} & =3 A^{\prime}+\lambda^{\prime} \frac{d}{d \lambda} \log \left(h(\lambda) V_{f 0}(\lambda)\right) ; & F_{2} & =\frac{2 a(\lambda) e^{2 A}}{h(\lambda)} ; \\
F_{3} & =h(\lambda) e^{-2 A}\left[4 A^{\prime}+\lambda^{\prime} \frac{d}{d \lambda} \log \left(\sqrt{h(\lambda)} V_{f 0}(\lambda)\right)\right] ; & F_{4} & =2 a(\lambda) ; \\
F_{5} & =-\lambda^{\prime} \frac{d a(\lambda)}{d \lambda} ; & F_{6} & =-e^{-2 A} h(\lambda) \lambda^{\prime} \frac{d a(\lambda)}{d \lambda}
\end{array}
$$

and the primes are derivatives with respect to $r$. Notice that the last two terms vanish if $a(\lambda)$ is constant.

We consider generic power-law asymptotics

$$
h(\lambda) \sim h_{0} \lambda^{-\rho} ; \quad a(\lambda) \sim a_{0} \lambda^{\sigma} ; \quad V_{f 0}(\lambda) \sim W_{0} \lambda^{\tau}
$$

of the potentials at large $\lambda$, and introduce a shorthand notation for the asymptotic behavior in (E.18)-(E.20):

$$
\begin{aligned}
A & =-\left(\frac{r-r_{0}}{R}\right)^{\alpha}+\frac{\alpha-1}{2} \log \frac{r-r_{0}}{R}+A_{c}+\mathcal{O}\left(\frac{r-r_{0}}{R}\right)^{-\alpha} \\
\log \lambda & =\frac{3}{2}\left(\frac{r-r_{0}}{R}\right)^{\alpha}+\lambda_{c}+\mathcal{O}\left(\frac{r-r_{0}}{R}\right)^{-\alpha}
\end{aligned}
$$

where we set $r_{0}=0$. Then the leading behavior of the coefficients $F_{i}$ at large $r$ is

$$
\begin{aligned}
& F_{1} \sim-\frac{3 \alpha(2+\rho-\tau)}{2 r}\left(\frac{r}{R}\right)^{\alpha} \\
& F_{2} \sim \frac{2 a_{0} e^{2 A_{c}+\rho \lambda_{c}+\sigma \lambda_{c}}}{h_{0}}\left(\frac{r}{R}\right)^{\alpha-1} \exp \left[\left(\frac{3}{2} \rho+\frac{3}{2} \sigma-2\right)\left(\frac{r}{R}\right)^{\alpha}\right] \\
& F_{3} \sim-\frac{h_{0} \alpha(16-6 \tau+3 \rho) e^{-2 A_{c}-\rho \lambda_{c}}}{4 R} \exp \left[\left(2-\frac{3}{2} \rho\right)\left(\frac{r}{R}\right)^{\alpha}\right] \\
& F_{4} \sim 2 a_{0} e^{\sigma \lambda_{c}} \exp \left[\frac{3 \sigma}{2}\left(\frac{r}{R}\right)^{\alpha}\right] \\
& F_{5} \sim-\frac{3 a_{0} \alpha \sigma e^{\sigma \lambda_{c}}}{2 r}\left(\frac{r}{R}\right)^{\alpha} \exp \left[\frac{3}{2} \sigma\left(\frac{r}{R}\right)^{\alpha}\right] \\
& F_{6} \sim-\frac{3 a_{0} h_{0} \alpha \sigma e^{-2 A_{c}-\rho \lambda_{c}+\sigma \lambda_{c}}}{2 R} \exp \left[\left(2+\frac{3}{2} \sigma-\frac{3}{2} \rho\right)\left(\frac{r}{R}\right)^{\alpha}\right] .
\end{aligned}
$$

For $\sigma=0$ the leading terms of $F_{5}$ and $F_{6}$, given above, become zero. If $a(\lambda)$ is constant for all $\lambda$, these terms actually vanish identically. If $a(\lambda)$ only asymptotes to a constant value, the leading behavior of $F_{5}$ and $F_{6}$ is determined by the next-to-leading term in the asymptotics of $a(\lambda)$. These will contribute in some particular cases, as we discuss below. 
- $\sigma>0$ and $\rho<4 / 3$. The tachyon EoM (E.27) is dominated by the terms $\propto T, T^{\prime} T^{2}$, which have the coefficients $F_{2}$ and $F_{5}$, respectively. Solving the tachyon from these terms gives the asymptotics

$$
T(r) \sim T_{0}-\frac{4 R e^{2 A_{c}+\rho \lambda_{c}}}{3 \alpha \sigma T_{0}} \int_{r}^{\infty} d \hat{r} \exp \left[\left(\frac{3}{2} \rho-2\right)\left(\frac{\hat{r}}{R}\right)^{\alpha}\right] .
$$

Substituting this back to the full equation of motion, we see that with the above constraints for $\sigma$ and $\rho$ it is indeed a solution. Further, the factor $\exp \left(-a(\lambda) T^{2}\right)$ vanishes double-exponentially, which confirms the decoupling of the tachyon from the other fields. In addition to the trivial reparametrization symmetry, the only free parameter of the asymptotic solution is $T_{0}$, which suggest that the singularity is of the "good" kind. However, tachyon solutions that are regular in the IR have bulk flavor anomalies which differ from those of QCD [91, 101, 102]. Therefore, we discard this option.

- $\sigma>0$ and $\rho=4 / 3$. The same terms continue to dominate, but the asymptotic changes. We find instead

$$
T(r) \sim 2 \sqrt{\frac{2 R}{3 \alpha \sigma h_{0}}} e^{A_{c}+\frac{2}{3} \lambda_{c}} \sqrt{r-r_{1}}
$$

where $r_{1}$ is a free parameter. One can again check that this is indeed a solution, and that the tachyon decouples from $A$ and $\lambda$. The terms proportional to $T^{\prime 2} T$ and $T^{\prime 3} T^{2}$ are suppressed only by $r^{-\alpha}$, but taking them into account results in a trivial factor multiplying the equation of motion, so that the solution in eq. (E.41) is unchanged for any value of $\alpha$. The asymptotics has only one free parameter, and the tachyon diverges as $r \rightarrow \infty$, so this solution is acceptable.

- $\sigma \leq 0$ and $\rho<4 / 3-\sigma$. We find two different cases. The asymptotics is qualitatively similar to (E.40), but arises in a slightly different way. The leading terms are those proportional to $T^{\prime}$ and $T$, corresponding to coefficients $F_{1}$ and $F_{2}$, respectively, as well as the double derivative term $T^{\prime \prime}$. This term being leading, one might expect that the asymptotics contains two free parameters, and is thus not of the good kind. This is indeed the case for $\tau>2+\sigma$. For $\tau \leq 2+\sigma$ only one parameter family of the asymptotic solutions is consistent with the other terms being subleading. However, since the tachyon becomes constant in the IR, we have the aforementioned problem with flavor anomalies, and hence we shall discard this solution in any case.

- $\sigma=0, \rho=4 / 3$, and $\tau<10 / 3$. The leading terms are typically those proportional to $T^{\prime 3}$ and $T^{\prime 2} T$. However, if $a(\lambda)$ is not constant, there is an extra constraint from the next-to-leading term in the expansion of $a(\lambda)$. If, for example, $a(\lambda)=$ $a_{0}+a_{1} / \lambda+\cdots$, we need to require $\alpha>1$. Assuming that all constraints are met, we find the exponential behavior

$$
T(r) \sim T_{0} e^{C r}
$$


where $T_{0}$ is a free parameter and

$$
C=\frac{4 e^{A_{c}+4 \lambda_{c} / 3} R a_{0}}{(10-3 \tau) \alpha h_{0}} .
$$

This special case is the asymptotics that was discussed in the probe limit in [71-73], and is acceptable. By inserting the expressions for $A_{c}$ and $\lambda_{c}$ from eqs. (E.18), (E.20), and (E.21), the coefficient simplifies to

$$
C=\frac{2^{2+P} 3^{2-P} a_{0}}{(10-3 \tau)(1-P) h_{0} V_{0} R} .
$$

- $\sigma=0, \rho=4 / 3$, and $\tau>10 / 3$. The leading terms are proportional to $T^{\prime}$ and $T$, which results in the tachyon vanishing asymptotically. In this case the factor $\exp \left(-a(\lambda) T^{2}\right)$ goes to one rather zero, which suggest that the correct physical picture, as discussed in the main text, cannot be achieved, even though the tachyon apparently decouples in the IR.

- $\sigma<0$ and $\rho=4 / 3-\sigma$. The leading terms are again proportional to $T^{\prime}$ and $T$. For $\tau<10 / 3-\sigma / 2$, the solution is exponentially increasing,

$$
T(r) \sim e^{C r}
$$

with

$$
C=\frac{4 e^{A_{c}+4 \lambda_{c} / 3} R a_{0}}{(10-3 \tau-3 \delta) \alpha h_{0}} .
$$

The factor $\exp \left(-a(\lambda) T^{2}\right)$ vanishes in the IR limit if $\alpha<1$, which is the expected behavior, so the solution is acceptable. For $\alpha>1$ or $\tau>10 / 3-\sigma / 2$ the factor goes to one instead, and it seems that the correct physical picture cannot be obtained. The borderline case $\alpha=1$ has either behavior depending on the values of other parameters.

In all the remaining cases, in particular for large $\rho$, the asymptotic solution of eq. (E.27) oscillates with increasing frequency as $r \rightarrow \infty$. Therefore, the tachyon is apparently not decoupled from the other fields, and the singularity discussed in the subsection does not exist.

In summary, we found good solutions only if $\rho=4 / 3$ and $\sigma \geq 0$, or $\rho=4 / 3-\sigma$ and $\sigma<0$. The latter case is included for $0<\alpha<1$, and the former case we found some extra constraints at the endpoint $\sigma=0$, which are detailed above. In the numerics we shall fix $P=1 / 2$ so that $\alpha=2$, and use potentials with $\rho=4 / 3$ and $\sigma \geq 0$, see appendix C.

\section{E.3 Singularity at the IR fixed point with $T \equiv 0$}

As discussed in the main text, some of the solutions with identically vanishing tachyon are expected to correspond to field theories where the chiral symmetry is conserved. Potentials for Yang-Mills theory were discussed extensively in [71-73, 76]. Therefore, we will only consider the case of potentials $V_{\text {eff }}(\lambda)=V_{g}(\lambda)-x V_{f}(\lambda, 0)$ which have a (single) maximum at some $\lambda=\lambda_{*}$, interpreted as an infrared fixed point. 
In the absence of the tachyon the space of solutions is one-dimensional. We identify two distinct one-parameter families of solutions: one where the solution bounces back to smaller couplings as the $\beta$-function $d \lambda / d A$ goes to zero (a special case of the bounce-back solutions discussed above in section E.1.2) and another where the $\beta$-function asymptotes as $d \lambda / d A \sim-3 \lambda$ (a special case of the solutions discussed in section E.2.1). These families are separated by a single solution where the $\beta$-function terminates at zero at the maximum of the potential $\lambda=\lambda_{*}$.

The limiting solution has a singularity at $r=\infty$ where $A$ diverges and $\lambda$ approaches $\lambda_{*}$ from below. We expand the potential around $\lambda=\lambda_{*}$ as

$$
V_{\text {eff }}(\lambda)=V_{0}+V_{2}\left(\lambda-\lambda_{*}\right)^{2}+\cdots
$$

where $V_{2}$ is negative. The equations of motion are solved by

$$
\begin{aligned}
& \lambda=\lambda_{*}-\left(\frac{r-r_{0}}{R}\right)^{-\delta}+\cdots \\
& A=-\log \left(r-r_{0}\right)+A_{0}+A_{1}\left(\frac{r-r_{0}}{R}\right)^{-2 \delta}+\cdots
\end{aligned}
$$

where $A_{0}$ and $\delta$ are related to the IR AdS radius and the derivative of the $\beta$-function, respectively, by

$$
\begin{gathered}
\ell_{\mathrm{IR}}^{2}=e^{2 A_{0}}=\frac{12}{V_{0}} \\
\lim _{r \rightarrow \infty} \frac{1}{\lambda-\lambda_{*}} \frac{\lambda^{\prime}(r)}{A^{\prime}(r)}=\delta=\sqrt{4-\frac{9 V_{2} \lambda_{*}^{2}}{V_{0}}}-2,
\end{gathered}
$$

and we also find

$$
A_{1}=-\frac{2 \delta}{9(2 \delta-1) \lambda_{*}^{2}} .
$$

\section{E.3.1 Generalization to $T \ll 1$}

A generalization of the $T \equiv 0$ case, which is of high interest to us, is where the tachyon mass and the condensate are very small in the UV, such that the tachyon remains small $(|T| \ll 1)$ even as $\lambda$ approaches the fixed point $\left(\lambda_{*}-\lambda \ll 1\right)$. However, for any nonzero tachyon profile, the tachyon will eventually become $\mathcal{O}(1)$ at some high $r=r_{\mathrm{IR}}$, and drive the flow away from the fixed point. Setting $r_{0}=0$, the above formulas (E.48) and (E.49) then hold in the limit $R \ll r \ll r_{\text {IR }}$ (with the understanding that depending on the value of $\delta$, the next-to-leading terms may be affected by the tachyon solution). This approximation is useful in the quasiconformal, or "walking" region of backgrounds for $x$ below, but close to the edge of the conformal window at $x_{c}$. Notice that $R$ is the scale where the coupling starts to deviate significantly from its fixed point value $\lambda_{*}$, and may be therefore identified as the UV scale of the theory. Therefore we shall denote $R=r_{\mathrm{UV}}$ in this case.

The tachyon profile can also be derived in this region. Inserting the solutions of eqs. (E.48) and (E.49) into the tachyon EoM (5.11), and taking $|T| \ll 1$, we get

$$
T^{\prime \prime}(r)+\frac{3}{r} T^{\prime}(r)+\frac{2 a\left(\lambda_{*}\right) \ell_{\mathrm{IR}}^{2}}{h\left(\lambda_{*}\right) r^{2}} T(r)=0 .
$$


There are two kinds of solutions depending on the value of the tachyon mass term. As in section 8.3 we can define the dimension $\Delta$

$$
\Delta(4-\Delta)=\frac{2 a\left(\lambda_{*}\right) \ell_{\mathrm{IR}}^{2}}{h\left(\lambda_{*}\right)}=\frac{24 a\left(\lambda_{*}\right)}{h\left(\lambda_{*}\right) V_{\mathrm{eff}}\left(\lambda_{*}\right)} .
$$

If the combination on the right hand side is less than 4 , which corresponds to $x>x_{c}$ we find two real roots $\Delta_{ \pm}$. In this case the tachyon solution is

$$
T(r) \sim T_{0}\left(\frac{r}{r_{\mathrm{IR}}}\right)^{\Delta_{-}}
$$

for $r_{\mathrm{IR}} \ll r \ll r_{\mathrm{IR}}$, where $\Delta_{-}$is the smaller root (unless we tuned the boundary conditions such that the quark mass is very small, in which case the solution with $\Delta_{-} \rightarrow \Delta_{+}$needs to be included).

For $x<x_{c}$ we have two complex roots $\Delta_{ \pm}=2 \pm i k$ instead, and the tachyon behaves as

$$
T(r) \sim T_{0}\left(\frac{r}{r_{\mathrm{IR}}}\right)^{2} \sin \left(k \log \frac{r}{r_{\mathrm{IR}}}+\phi\right) .
$$

This oscillating solution is the root of the rich structure of backgrounds found for $x<x_{c}$ in section 8 , which are also discussed below in appendix F.

\section{F Structure of the background as a function of $x$ and $T_{0}$}

In this appendix we will explain in detail how the phase structure of the background solutions seen in figure 5, and in particular the region with nearly conformal behavior, arises.

The structure in the plots is linked to the transition of the system from the UV region, where the tachyon is small and the background is characterized by the potential $V_{\text {eff }}(\lambda)=$ $V_{g}(\lambda)-x V_{f 0}(\lambda)$, to the IR region, where the tachyon is large, and the background is characterized by $V_{g}(\lambda)$. First, recall that $V_{\text {eff }}(\lambda)$ has a maximum at some $\lambda=\lambda_{*}$ which depends on $x$. For $x \rightarrow 0$ we find from eqs. (8.24) and (8.26) that $\lambda_{*} \rightarrow \infty$, whereas for $x \rightarrow 11 / 2$ we obtain $\lambda_{*} \rightarrow 0$. This maximum suggests a presence of an IR fixed point of the $\beta$-function for the coupling $\lambda$.

However, for a nontrivial tachyon profile the fixed point is not reached. When approaching $\lambda=\lambda_{*}$ from the UV, the solution is driven away from the fixed point as soon as the tachyon becomes large, $T \sim \mathcal{O}(1)$, and the system enters the region where all EoMs are nontrivially coupled. The point where this happens, is controlled by the normalization of the tachyon in the IR, i.e., the value of $T_{0}$ (Assuming potentials of scenario I). The larger $T_{0}$, the smaller is the value of $\lambda$ where the tachyon decouples.

The solid blue curve in figure 5 is the critical value of $T_{0}$ where the tachyon decouples at $\lambda=\lambda_{*}$. Actually, precisely at this curve the UV asymptotics is that of section D.2, and the solution ends at the fixed point. If $T_{0}$ is smaller than the critical value, the tachyon will become small $\ll 1$ during the flow toward the UV when we still have $\lambda>\lambda_{*}$, so that the $\beta$-function corresponding to the effective potential $V_{\text {eff }}$ is positive. As the tachyon decouples, the $\beta$-function flow approaches that defined by $V_{\text {eff }}$. Therefore, to the the region 
with "standard" UV behavior is not reached at all, but the solution bounces back at a finite value of the coupling (see also figure 17 below) where the $\beta$-function (evaluated along the $\mathrm{RG}$ flow) crosses zero. The strong dependence of the blue curve on $x$ is explained by the dependence of $\lambda_{*}$ on $x$ : for example as $x \rightarrow 0$, the fixed point moves to large values of $\lambda_{*}$, and the critical tachyon IR value $T_{0}$ required for avoiding the bounce-back approaches zero.

The argument above is not rigorous, as it involves the location of the decoupling of the tachyon which is not defined precisely. However, one should notice that as the blue curve is approached from above, the system is on the verge of reaching an IR fixed point so that the coupling freezes, i.e., it evolves very slowly for a large range of $r$. Meanwhile, the tachyon grows relatively fast with $r$. Therefore, the value of $\lambda$ where the tachyon decoupling takes place becomes more and more precisely defined as the blue curve is approached from above. This can also be seen in the numerical examples below and in section 8 .

To understand how the red dashed curve arises, we need to study the tachyon solutions in the UV region. Below the red curve, the tachyon solution develops a zero, as is required by the negative value of the quark mass. This zero actually appears in the region, where (the absolute value of) the tachyon is still small. If we continue on the plot toward lower values of $T_{0}$, the quark mass becomes again zero, and then positive extremely close to the solid blue curve. This happens as the tachyon develops a second zero in the UV. We can continue further, and find solutions with an arbitrary number $n$ of zeroes (which are very hard to construct numerically). See figure 6 (left) in the main text for the qualitative behavior of the quark mass as the blue curve is approached.

The oscillating behavior of the tachyon in the UV is linked to the violation of the BF bound: when $\Delta_{\mathrm{IR}}\left(4-\Delta_{\mathrm{IR}}\right)$ is smaller than 4 , the solutions for $\Delta_{\mathrm{IR}}$ are complex, which results in the oscillations of the tachyon solution. We plotted the squared mass of the tachyon at the IR fixed point in figure 2, where the solid thick blue curve corresponds to the present choice of parameters.

As we approach the solid blue curve from within the contoured region in figure 5, the background can be approximated near the fixed point as discussed above in section E.3.1. By the definition of eq. (8.17), the BF bound is violated at the fixed point for $x<x_{c}$. Therefore, as we approach the blue curve from above in this region and the system is about to develop a fixed point, the tachyon necessarily oscillates as soon as values of $\lambda$ close enough to $\lambda_{*}$ are reached. In this case, the tachyon is well approximated by the solution of eq. (E.56),

$$
T(r) \sim T_{0}\left(\frac{r}{r_{\mathrm{IR}}}\right)^{2} \sin \left(k \log \frac{r}{r_{\mathrm{IR}}}+\phi\right),
$$

where $k$ is fixed by the potentials,

$$
k=\sqrt{\frac{24 a\left(\lambda_{*}\right)}{h\left(\lambda_{*}\right) V_{\mathrm{eff}}\left(\lambda_{*}\right)}-4},
$$

but $\phi$ and $T_{0}$ are free parameters, which will be fixed by the boundary conditions. For $x>x_{c}$, the $\mathrm{BF}$ bound is not violated at the fixed point, and therefore no oscillations are 
expected, and the tachyon dependence is as in eq. (E.55). The (uppermost) curve of zero quark mass (the red dashed one in the plots) essentially limits the region of oscillating tachyon: for example on the left hand plot above this curve quark mass is positive (no tachyon zeroes) and below it the mass is negative (one tachyon zero), see the mass dependence in figure 6 . Notice that the red curve must therefore join the blue curve at $x=x_{c}$.

As we approach the fixed point keeping $x$ fixed, the range $r_{\mathrm{UV}} \ll r \ll r_{\text {IR }}$ where eq. (F.1) holds grows without limit. ${ }^{29}$ Here $r_{\mathrm{UV}}$ is the scale where the coupling starts to deviate significantly from its fixed point value $\lambda_{*}$ as we follow the flow toward the UV. According to eq. (F.1), the number of encountered tachyon zeroes is

$$
\pi n \sim k \log \frac{r_{\mathrm{IR}}}{r_{\mathrm{UV}}} .
$$

We may take one step further, and find the scaling of the quark mass and the chiral condensate as $r_{\mathrm{IR}} / r_{\mathrm{UV}} \rightarrow \infty$ and $n \rightarrow \infty$. This can be done by matching the "intermediate" tachyon solution (F.1) with the UV (and IR) solutions (see section 10 and appendix $\mathrm{H}$ where we do the matching procedure more carefully for $x \rightarrow x_{c}$ ). The tachyon is supposed to become large at $r \sim r_{\mathrm{IR}}$, so $T_{0} \sim 1$. On the other hand at $r \sim r_{\mathrm{UV}}$ we enter the standard UV region, where roughly $T \sim m r+\sigma r^{3}$. Matching in the UV gives the typical sizes for $m$ and $\sigma$ for large $n$ :

$$
m r_{\mathrm{UV}} \sim \sigma r_{\mathrm{UV}}^{3} \sim\left(\frac{r_{\mathrm{UV}}}{r_{\mathrm{IR}}}\right)^{2} \sim \exp \left(-\frac{2 \pi n}{k}\right) .
$$

In particular, the maximal masses for which solutions with $n$ tachyon nodes exist, or in other words the sizes of the bumps in figure 6 (left), numbered from right to left, must obey this scaling law. As the mass scale vanishes exponentially for $n \rightarrow \infty$, for any fixed $m \neq 0$ there are only finite number of backgrounds as $n$ is limited from above, whereas for $m=0$ we find an infinite tower of solutions.

At this point is appropriate to remind the reader that the solution with no tachyon nodes $(n=0)$ always has smaller free energy than the solutions with $n>0$ (see section 9). Also, the $n=0$ solution is not found in the scaling region where the system is close to having a fixed point in general. For $m=0$ this solution (red dashed curve of figure 5 enters the scaling region (which is close to the solid blue curve in the same figure) only in the limit $x \rightarrow x_{c}$, which will be discussed in detail in section 10 .

We conclude with one more observation on the location of the curve of the vanishing quark mass. The discussion above was relying on the dimension $\Delta_{\text {IR }}$ at the fixed point, and

\footnotetext{
${ }^{29}$ We cannot prove analytically that the scaling region is accessible, because this would require a description of the solution in the IR region where tachyon is not decoupled. However, we may solve the EoMs starting from the UV with arbitrary small quark masses and vevs, which are guaranteed to enter the oscillating region, and have arbitrary many tachyon zeroes. The tricky issue is, if we can tune the solutions such that it ends in the good IR singularity after the tachyon finally grows large and the oscillations end in the IR. Due to the oscillating nature of the solution, it is plausible that the good IR singularity is found after any number of oscillations $n$, if it is found, e.g., for small $n$. In the end, this question is settled by the numerics, which supports our expectations, and solutions with regular IR behavior are indeed found for any $n$.
} 
therefore we could argue how the tachyon behaves in the limit where the background comes arbitrarily close to the fixed point, i.e., as we approach the blue solid curve from above in figure 5. However, we can also present rough qualitative arguments on the behavior of the curve further away from the solid blue curve. Because the tachyon is in any case small in the interesting region, we can read directly from the linearized tachyon EoM whether it oscillates or not. The EoM reads

$$
T^{\prime \prime}(r)+\left[3 A^{\prime}+\lambda^{\prime} \frac{d}{d \lambda} \log \left(h(\lambda) V_{f 0}(\lambda)\right)\right] T^{\prime}(r)+\frac{2 a(\lambda) e^{2 A}}{h(\lambda)} T(r) \simeq 0 .
$$

Recall that the tachyon is decoupled in this region and evolves independently of the other fields. Assuming decoupling, the coefficients are essentially independent of $T_{0}$ and can be solved directly from the potential $V_{\text {eff }}(\lambda)=V_{g}(\lambda)-x V_{f 0}(\lambda)$. A is quite well approximated by $A \simeq-\log r,{ }^{30}$ the second term in the square brackets of the coefficient of $T^{\prime}$ is small, and therefore the essential term is, as in the case of the fixed point, the ratio $a(\lambda) / h(\lambda)$, which increases with $\lambda$. As $\lambda$ grows, at some critical $\lambda_{c}$ the ratio becomes large enough, and the tachyon starts to oscillate. In terms of the $\gamma$-function, this means that $\gamma / T$ reaches the value of approximately -2 . The oscillations do not take place if the tachyon grows large already for $\lambda<\lambda_{c}$ so that nonlinear terms contribute in eq. (F.5). Therefore, for the limiting solutions, the tachyon becomes $\mathcal{O}(1)$ roughly at $\lambda=\lambda_{c}$. As the start of the oscillations means that the quark mass goes to zero, this mechanism also fixes the location of the curve with zero quark mass (red dashed curve in figure 5).

In summary, the solid blue curve of figure 5 is stabilized by the tachyon growing large at $\lambda=\lambda_{*}$, whereas the dashed red curve is stabilized by the tachyon growing large at $\lambda=\lambda_{c}$. The two curves meet when $\lambda_{c}=\lambda_{*}$, which gives an alternative way to formulate the definition of $x_{c}$.

It is interesting to compare the picture in our model to that arising in the DysonSchwinger approach in the rainbow approximation (see, e.g., $[116,117]$ ). Also in this framework it is useful to define two values of the coupling, corresponding to $\lambda_{*}$ and $\lambda_{c}$ above. The definitions are similar as here: $\lambda_{*}$ is the zero of the $\beta$-function, and $\lambda_{c}$ is the value of $\lambda$ where the anomalous dimension of the chiral condensate reaches unity (so that $\Delta$ of section 8.3 equals two).

Indeed the latter definition corresponds to saturating the BF bound in the present approach, which in the vicinity of the IR fixed point means the start of the tachyon oscillations, matching with our definition of $\lambda_{c}$. Similarly as in our model, $\lambda_{c}=\lambda_{*}$ at the edge of the conformal window. A similar description of the conformal transition was also found in the holographic model of [15].

\section{F.1 Numerical analysis}

We illustrate the analysis above by studying the background numerically near the red dashed and blue solid curves of figure 5 .

\footnotetext{
${ }^{30}$ Explicit expressions can be derived in some approximation schemes, e.g., as series in the limit of small $\beta$-function.
} 

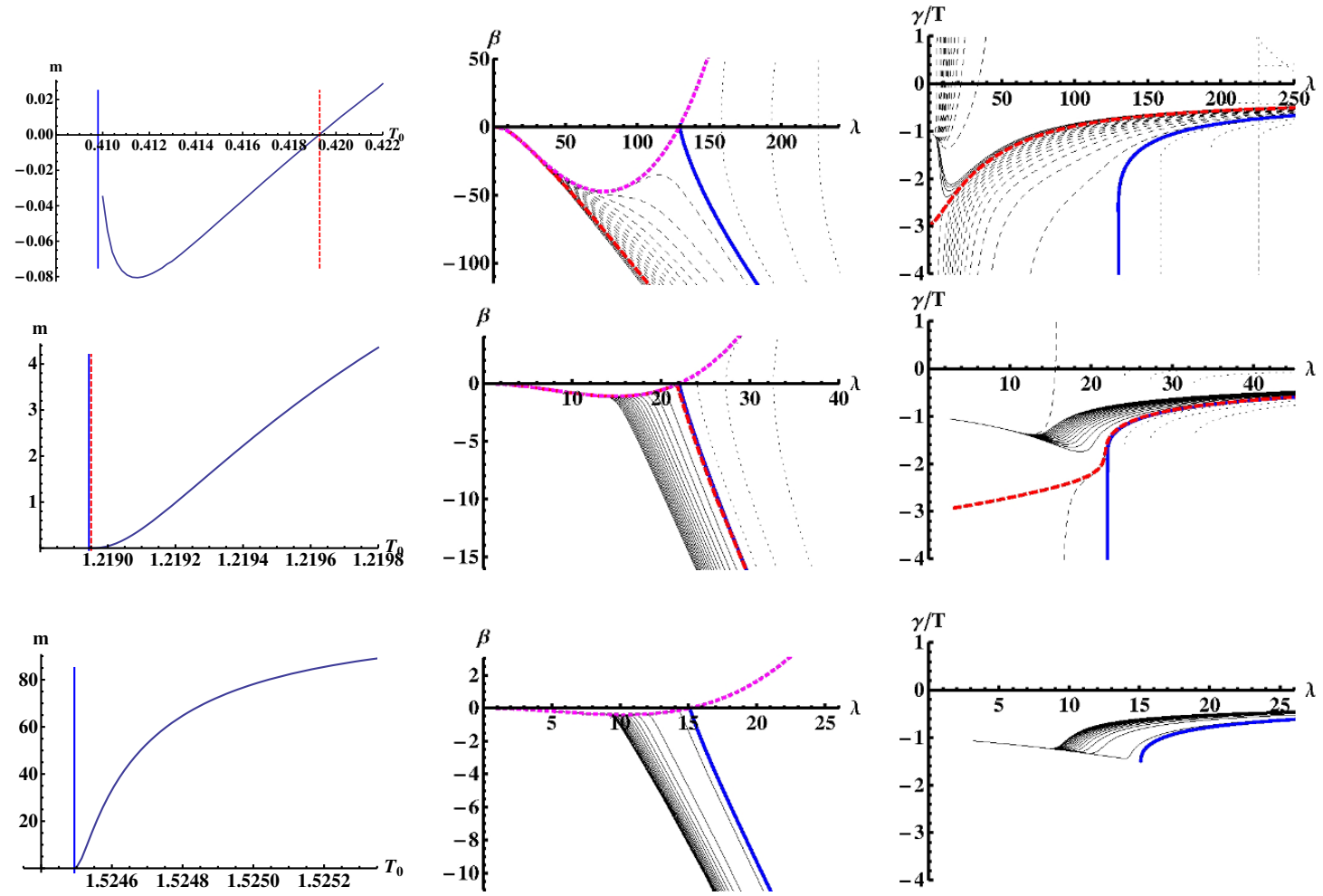

Figure 17. The dependence of the background on $T_{0}$ near the red and blue lines of figure 5 . Top row: $x=2$. Middle row: $x=3.9$. Bottom row: $x=4.25$. Left column: the quark mass as a function of $T_{0}$. The vertical blue solid and dashed red lines mark the solutions terminating at an UV fixed point, and having $m=0$, respectively, as in figure 5. Middle column: $\beta$-functions $d \lambda / d A$ as $T_{0}$ is varied over the range of the mass plots (left) with constant steps for $T_{0}$, shown as thin black curves of various kinds. Dotted, dashed and solid curves are $\beta$-functions for backgrounds with a bounce-back towards the IR, $m<0$, and $m>0$, respectively. The limiting cases between these behaviors are the given by the thick blue solid curves, which terminate at an UV fixed point, and the thick red dashed ones, which have $m=0$. The magenta dotted curve is the $\beta$-function when the tachyon is completely decoupled, solved from eq. (8.19). Right column: $\gamma$-functions $1 / T \times d T / d A$ as $T_{0}$ is varied. The lines are marked as for the $\beta$-functions.

We choose as reference values $x=2,3.9$ and 4.25 , which have qualitatively different behavior. For zero mass, they correspond to field theories with running, walking and IR conformal behavior of the coupling constant. Figure 17 shows, for the above values of $x$, the quarks mass in IR units (left column), the variation of the $\beta$-function (middle column), and the variation of the $\gamma$-function (right column) as we scan over a range of $T_{0}$ which includes the blue and red dashed curves of figure 5 .

For $x=2$ (top row) the quark mass (left column) is negative for a wide range of $T_{0}$ between the zero mass solution (dashed red vertical line) and the critical value of $T_{0}$ where the UV behavior of the solution changes such that the mass is no longer defined (solid blue vertical line). The mass curve oscillates as we approach the vertical blue line of critical $T_{0}$ as depicted in figure 6 , but the oscillations lie too close to line to be resolved at the 
used scale of $T_{0}$. As $x$ is increased to 3.9 (middle row), the zero mass solution is driven very close to the critical $T_{0}$. For $x=4.25$ (bottom row), the solutions with negative quark mass, and in particular the one with zero mass, have disappeared.

In the the middle column we plot as the thin black curves the $\beta$-function as $T_{0}$ is varied over the range of the left hand plots with a constant step size. The blue thick curve is the limiting $\beta$-function that always terminates at an UV fixed point and corresponds to the blue curve of figure 5. For $x=2$ all $\beta$-functions with positive quark mass (black thin solid curves) are running, including the zero mass solution (red dashed thick curve), whereas all walking solutions have negative quark mass (thin dashed curves). ${ }^{31}$ The running curves are the lowest ones and all of them basically overlap. The dotted thin lines are the $\beta$-functions with bounce-back behavior in the UV, which occur for values of $T_{0}$ that are smaller than the critical one. Increasing $x$ to 3.9 (middle row), the zero mass solution moves into the walking region, and approaches the critical one marked with thick blue curve. As $x$ is further increased (bottom row), the zero mass solution disappears by joining the blue curve.

Similar, but slightly more complicated behavior is seen for the $\gamma$-functions (right column). The dashing and coloring have the same meaning as for the $\beta$-functions. Notice that $\gamma / T$ asymptotes to -3 as $\lambda \rightarrow 0$ for the zero mass solution (red thick dashed curve) whereas for the solutions with a finite mass it asymptotes to -1 , as expected. The solutions with negative quark mass (thin dashed curves) have a zero of the tachyon, which shows up as a pole in $\gamma / T$. The main change in the plots as $x$ increases from 2 (top) to 4.25 (bottom) is the movement of the solution with the UV fixed point (blue thick curve) towards smaller $\lambda$, as all solutions it "crosses" change drastically. Otherwise the $\gamma$-functions (i.e., those solutions left of the blue curve) are roughly independent of $x$.

Finally, we comment on the $m \rightarrow 0$ limit and discuss also figure 12 in this same limit. For $x<x_{c}$ the background converges smoothly to the $m=0$ one with chiral symmetry breaking in this limit, as also seen from the $\beta$ - and $\gamma$-functions on figure 17 (top and middle rows). Taking $m \rightarrow 0$ for $x<x_{c}$ in figure 12 defines the enveloping curve of the fixed mass curves, which diverges for $x=x_{c}$. (We shall discuss the $m=0$ case in more detail in section 10 below.) For $x>x_{c}$, the scale ratio $\Lambda_{\mathrm{UV}} / \Lambda_{\mathrm{IR}}$ diverges for $m \rightarrow 0$ (see figure 12), and we can consider two different limits. If we keep $\Lambda_{\mathrm{UV}}$ fixed as $m \rightarrow 0$, the IR scale is driven to infinity and the background converges pointwise to the one with identically vanishing tachyon and an IR fixed point, discussed in section 8.5.1. If we instead keep the IR scale fixed, the background converges towards the one having an UV fixed point of section D.2 (see the $\beta$ - and $\gamma$-functions shown with the thick blue line on the bottom row of figure 17). Notice that the backgrounds with vanishing tachyon and $x<x_{c}$ are not connected to the backgrounds having a finite quark mass by any limiting procedure, which is in line with our expectation that they are unphysical. Indeed we shall show in section 9 that these solutions have larger free energy than the ones with nontrivial tachyon and chiral symmetry breaking.

\footnotetext{
${ }^{31}$ As mentioned above, there are also positive quark mass solutions which basically overlap with the thick blue curve. Our resolution is not enough to resolve these.
} 


\section{G Extracting UV coefficients from numerical solutions}

\section{G.1 Extracting free energy differences}

As pointed out in section 8 , we need to check numerically which one of the two solutions with vanishing quark mass, the one with chiral symmetry breaking or without, minimizes the free energy. In order to do this we need to extract $\hat{A}$ (for fixed $\Lambda$ ) defined in eq. (9.9) for both solutions and then calculate the energy difference through eq. (9.10).

The corrections involving $\hat{A}$ are highly suppressed $\sim r^{4}$ in the region which is under perturbative control $(\log (r \Lambda)$ is small). Extracting $\hat{A}$ directly from the numerical solutions of $A$ and $\lambda$ is practically impossible, since it is difficult to require the two solutions to have the same $\Lambda$ to a high enough precision. Therefore we study variations in $X$, which is invariant in scalings of $r$. In principle we could match the numerically extracted variation of $X$ to the correction term in eq. (9.14). This is doable (except for values of $x$ very close to the critical one $x_{c} \simeq 3.9959$ ), but a large uncertainty in the value of $\hat{A}$ still remains. Therefore we proceed as follows. We substitute $X_{0}+X_{1}$ in the equation (8.19), where $X_{1}$ is treated as a small perturbation. From the linearized equation, we can solve $X_{1}$ exactly:

$$
X_{1}(\lambda)=X_{C} \exp \left[\int_{1}^{\lambda} \frac{16 V(\hat{\lambda}) X_{0}(\hat{\lambda})^{3} / \hat{\lambda}+3 V^{\prime}(\hat{\lambda})+3 X_{0}(\hat{\lambda})^{2} V^{\prime}(\hat{\lambda})}{6 V(\hat{\lambda}) X_{0}(\hat{\lambda})^{2}} d \hat{\lambda}\right]
$$

where $X_{C}$ is a constant. So, if $X_{0}$ is known, this equation gives $X_{1}$ in eq. (9.12) to all orders in $\log (r \Lambda)$. Since the UV expansion of $X_{0}$ is also known, we can use that to expand $X_{1}$ and to calculate the relation between the constants $\hat{A}$ and $X_{C}$ by using eq. (9.13). A straightforward calculation gives

$$
\begin{aligned}
\hat{A}= & \frac{3 V_{1} \Lambda^{4} X_{C}}{20} \exp \left[-\frac{\log \left(9 V_{1} / 8\right)\left(23 V_{1}^{2}-64 V_{2}\right)}{9 V_{1}^{2}}\right] \\
& \times \exp \left\{-\int_{0}^{1}\left[\frac{16 V(\hat{\lambda}) X_{0}(\hat{\lambda})^{3} / \hat{\lambda}+3 V^{\prime}(\hat{\lambda})+3 X_{0}(\hat{\lambda})^{2} V^{\prime}(\hat{\lambda})}{6 V(\hat{\lambda}) X_{0}(\hat{\lambda})^{2}}\right.\right. \\
& \left.\left.-\left(\frac{32}{9 V_{1} \hat{\lambda}^{2}}+\frac{14}{9 \hat{\lambda}}-\frac{64 V_{2}}{9 V_{1}^{2} \hat{\lambda}}\right)\right] d \hat{\lambda}+\frac{32}{9 V_{1}}\right\}
\end{aligned}
$$

To obtain the free energy difference between two solutions, we calculate numerically the variation of $X$ and match with eq. (G.1), where we use either of the two solutions to evaluate $X_{0}$ and the integral numerically. Since $X_{1}$ given by eq. (G.1) is a good approximation already at small $r\left(\log (r \Lambda)\right.$ does not need to be small) we can obtain the difference $\Delta X_{C}$ between the solutions to a good precision. The value is then used to calculate numerically $\Delta \hat{A}$ and $\Delta \mathcal{E}$ through equations (9.10) and (G.2).

\section{G.2 Extracting the chiral condensate at $m=0$}

Let us then discuss how the value of the chiral condensate can be extracted from a numerical solution to the differential equations. Recall that our method for constructing the backgrounds requires tuning the normalization of the tachyon in the IR $\left(T_{0}\right)$ such that the 
quark mass vanishes. This procedure is however limited by the numerical precision of the solution, and therefore exactly zero quark mass cannot be obtained. As it turns out, this makes direct extraction of the condensate value difficult, since the approximately linear term $\sim m r$ of the tachyon dominates over the cubic solution $\sim \sigma r^{3}$ in the deep UV. This is particularly problematic as $x$ approaches $x_{c}$, since the ratio of the UV and IR energy scales grows, pushing the asymptotic UV region to smaller $r$ while the condensate value $\propto \sigma$ decreases.

Therefore, we employ a subtraction procedure. In the UV, the tachyon solution (with the mass $m_{1}$ as small as can be achieved) can be written as

$$
T_{1}(r)=m_{1} T_{m}(r)+\sigma_{1} T_{\sigma}(r) .
$$

The UV expansions of the two (approximately) linearly independent solutions $T_{m} \simeq$ $\ell r(-\log (\Lambda r))^{C}$ and $T_{\sigma}(r) \simeq \ell r^{3}(-\log (\Lambda r))^{-C}$ are given in appendix D.1.1. We will remove the remaining mass term by subtracting another solution

$$
T_{2}(r)=m_{2} T_{m}(r)+\sigma_{2} T_{\sigma}(r)
$$

which is calculated at the same value of $x$ but for different $T_{0}$ such that $m_{2}$ is about the same order as $m_{1}$. Since the mass terms dominate at very small $r$, it is easy to extract $m_{2} / m_{1}$ to a high precision, and construct ${ }^{32}$

$$
T_{\delta}(r)=T_{1}(r)-\frac{m_{1}}{m_{2}} T_{2}(r)=\left(\sigma_{1}-\frac{m_{1} \sigma_{2}}{m_{2}}\right) T_{\sigma}(r) .
$$

If $\sigma$ is analytic at $m=0$,

$$
\sigma_{i}=\sigma^{(0)}+\sigma^{(1)} m_{i}+\sigma^{(2)} m_{i}^{2}+\cdots
$$

we obtain

$$
T_{\delta}(r)=\left[\sigma^{(0)}\left(1-\frac{m_{1}}{m_{2}}\right)+\sigma^{(2)} m_{1}\left(m_{1}-m_{2}\right)+\cdots\right] T_{\sigma}(r) .
$$

If the masses $m_{i}$ are sufficiently small, the quadratic correction $\propto \sigma^{(2)}$ as well as higher order terms can be neglected, and the difference $T_{\delta}$ is proportional to $\sigma^{(0)}$ to a good precision. The reliability of the subtraction procedure can be tested by varying, say, $m_{2}$ and checking that this does not affect the result.

Now $\sigma^{(0)}$ can extracted from $T_{\delta}$ in a straightforward manner. However the result still has quite large error bars in particular for $x$ close to $x_{c}$, because of numerical uncertainty in the deep UV. We do an additional trick to remove this problem. The extracted $T_{\delta}$ extends close enough to the UV singularity to ensure that the tachyon is decoupled from $A$ and $\lambda$. On the other hand, we can obtain a decoupled tachyon solution with zero quark mass by solving first the background functions $A$ and $\lambda$ with $T \equiv 0$, inserting these in the tachyon EoM, and solving that by shooting from the UV. We can basically extend this solution as

\footnotetext{
${ }^{32}$ There is a subtlety here: the UV scales $\Lambda$ which appear in eq. (D.9) will be different for the two solutions. This needs to be fixed, e.g., by scaling one of the solutions such that the corresponding solutions for $\lambda$ match as $\lambda \rightarrow 0$.
} 
close to the UV singularity as we want. Matching the two solutions in the region where both are reliable, we can extend $T_{\delta}$ easily up to $r \sim 10^{-100}$ in the UV.

Finally, to extract $\sigma^{(0)}$ we consider the expansion of the tachyon at small $\lambda$ :

$$
\begin{gathered}
\log T_{\delta}(\lambda)-\log \left(1-\frac{m_{1}}{m_{2}}\right)-\log \ell \simeq \log \sigma^{(0)}+\log T_{\sigma}(\lambda)-\log \ell \\
=\log \frac{\sigma^{(0)}}{\Lambda^{3}}-\frac{8}{3 V_{1} \lambda}+\left(\frac{4}{3} \frac{h_{1}}{V_{1}}+\frac{39 V_{1}^{2}-64 V_{2}}{12 V_{1}^{2}}\right) \log \frac{9 V_{1} \lambda}{8}+\cdots,
\end{gathered}
$$

which is obtained by using eqs. (D.9) and (D.2). The coefficients $V_{i}$ and $h_{1}$ were defined in eqs. (D.4), and $\Lambda=\Lambda_{\mathrm{UV}}$ is the scale of the UV expansions. We match this expansion with the extended $T_{\delta}$, and extrapolate to $\lambda=0$ to obtain $\log \left(\sigma^{(0)} / \Lambda^{3}\right)$.

\section{H Details on BKT scaling}

In this appendix we discuss some technical details on the BKT scaling. First, there is a subtlety with matching the approximation (10.11) with the tachyon solution in the IR (for which we were unable to write analytic approximations). Since the approximation contains a periodic function, the boundary conditions at $r \simeq r_{\text {IR }}$ will not fix the tachyon uniquely: one can let the tachyon oscillate first, and do the matching only after the oscillations have created $n$ zeroes, where $n=0,1,2, \ldots$ Therefore, in eq. (10.15) we should actually have (including the solutions where the tachyon changes sign)

$$
\sqrt{\kappa\left(\lambda_{*}-\lambda_{c}\right)} \log \frac{r_{\mathrm{IR}}}{\hat{r}} \sim \pi n+\mathcal{O}(1) .
$$

An analogous result was found in [15] in a case that was analytically more tractable. As in their case, we expect that the solution with no nodes $(n=0)$ is the one that minimizes the free energy, as we already verified numerically in section 9 .

One can actually do the matching procedure even more precisely, and in particular derive results for $K$ and $\hat{K}$. We need to study the approximation (10.11) more closely: actually it can be matched with the other solutions only in the vicinity of the zeroes of the sine function. At $r=r_{\mathrm{IR}}$ we have

$$
\begin{aligned}
T\left(r_{\mathrm{IR}}\right) \simeq & C_{\mathrm{fp}}\left(\frac{r_{\mathrm{IR}}}{r_{\mathrm{UV}}}\right)^{2} \sin \left(\sqrt{\kappa\left(\lambda_{*}-\lambda_{c}\right)} \log \frac{r_{\mathrm{IR}}}{\hat{r}}+\hat{\phi}\right) \\
T^{\prime}\left(r_{\mathrm{IR}}\right) \simeq & C_{\mathrm{fp}} \frac{r_{\mathrm{IR}}}{r_{\mathrm{UV}}^{2}}\left[2 \sin \left(\sqrt{\kappa\left(\lambda_{*}-\lambda_{c}\right)} \log \frac{r_{\mathrm{IR}}}{\hat{r}}+\hat{\phi}\right)\right. \\
& \left.+\sqrt{\kappa\left(\lambda_{*}-\lambda_{c}\right)} \cos \left(\sqrt{\kappa\left(\lambda_{*}-\lambda_{c}\right)} \log \frac{r_{\mathrm{IR}}}{\hat{r}}+\hat{\phi}\right)\right] .
\end{aligned}
$$

It appears that the cosine factor in the derivative can be neglected due to the small factor $\sqrt{\kappa\left(\lambda_{*}-\lambda_{c}\right)}$ multiplying it. However, in this case the ratio $T\left(r_{\mathrm{IR}}\right) / T^{\prime}\left(r_{\mathrm{IR}}\right)=1 / 2 r_{\mathrm{IR}}$ is fixed so we cannot match both the function and its derivative with the (nontrivial) IR part of the tachyon solution. Therefore, we indeed need to be close to a zero of the sine,

$$
\sin \left(\sqrt{\kappa\left(\lambda_{*}-\lambda_{c}\right)} \log \frac{r_{\mathrm{IR}}}{\hat{r}}+\hat{\phi}\right)=\mathcal{O}\left(\sqrt{\lambda_{*}-\lambda_{c}}\right),
$$

which brings in the required dependence of the solution on both $C_{\mathrm{fp}}$ and $\phi$. 
Matching the solution (10.11) towards the UV is only possible close to its node, as well. ${ }^{33}$ This gives

$$
\hat{\phi}=\mathcal{O}\left(\sqrt{\lambda_{*}-\lambda_{c}}\right) .
$$

Consequently, the argument of the sine in the approximation (10.11) must change by a positive multiple of $\pi$ as we move from the UV to the IR, so that eq. (H.1) is actually written as

$$
\sqrt{\kappa\left(\lambda_{*}-\lambda_{c}\right)} \log \frac{r_{\mathrm{IR}}}{\hat{r}} \simeq \pi(n+1) .
$$

where $n$ is the number of the zeroes of the tachyon solution. ${ }^{34}$ Notice that eqs. (10.13) and (10.14) also receive extra factors from the refined scaling argument. We have now

$$
C_{\mathrm{fp}} \sim \sigma r_{\mathrm{UV}}^{3} \frac{1}{\sqrt{\lambda_{*}-\lambda_{c}}} \sim\left(\frac{r_{\mathrm{UV}}}{r_{\mathrm{IR}}}\right)^{2} \frac{1}{\sqrt{\lambda_{*}-\lambda_{c}}}
$$

instead of eq. (10.14). The additional square root factors cancel in the result for $\sigma$, and they could in any case be neglected as a subleading correction to the exponential scaling.

In order to write down the results for $K$ and $\hat{K}$ we recall that the definitions of $\lambda_{c}(x)$ and $x_{c}$ read

$$
G\left(\lambda_{c}(x), x\right)=4 ; \quad G\left(\lambda_{*}\left(x_{c}\right), x_{c}\right)=4,
$$

respectively, where $\lambda_{*}(x)$ was defined by $V_{g}^{\prime}\left(\lambda_{*}(x)\right)-x V_{f 0}^{\prime}\left(\lambda_{*}(x)\right)=0$. Near the critical point we have the two expansions

$$
G\left(\lambda_{*}, x\right) \simeq 4+\frac{\partial}{\partial \lambda} G\left(\lambda_{c}, x\right)\left(\lambda_{*}-\lambda_{c}\right) \simeq 4+\left.\frac{d}{d x} G\left(\lambda_{*}(x), x\right)\right|_{x=x_{c}}\left(x-x_{c}\right)
$$

where $\kappa=\frac{\partial}{\partial \lambda} G\left(\lambda_{c}, x\right)$. By using eq. (H.5) we finally see that

$$
\frac{r_{\mathrm{IR}}}{r_{\mathrm{UV}}} \sim \exp \left[\frac{K(n+1)}{\sqrt{\lambda_{*}-\lambda_{c}}}\right] \sim \exp \left[\frac{\hat{K}(n+1)}{\sqrt{x_{c}-x}}\right]
$$

where

$$
K=\frac{\pi}{\sqrt{\kappa}}=\frac{\pi}{\sqrt{\frac{\partial}{\partial \lambda} G\left(\lambda_{c}, x\right)}} ; \quad \hat{K}=\frac{\pi}{\sqrt{-\left.\frac{d}{d x} G\left(\lambda_{*}(x), x\right)\right|_{x=x_{c}}}},
$$

and setting $n=0$ gives the result for the solution which has no tachyon zeroes and lowest free energy.

\footnotetext{
${ }^{33}$ Proving this turns out to be more tricky than in the IR, since the solutions (10.10) and (10.11) have the same power behavior, and therefore they apparently join smoothly for large values of the sine function. However, this is a fake effect due to the roughness of the approximation (10.10): we have seen that in the UV the power law of the tachyon actually changes from 2 to 3 within a range of $\log r$ which is small with respect to $1 / \sqrt{\lambda_{*}-\lambda_{c}}$. Therefore the same argument as in the IR applies, and the sine function needs to be $\mathcal{O}\left(\sqrt{\lambda_{*}-\lambda_{c}}\right)$ in order the matching to work.

${ }^{34}$ Our arguments do not exclude zeroes of the tachyon solution near the endpoints of the validity of the approximation (10.11), i.e., near $r=\hat{r}$ and $r=r_{\mathrm{IR}}$. However it is reasonable to expect that the approximation joins the UV and IR solutions in a smooth manner, so that no extra nodes appear, which can also be verified numerically.
} 
Finally, let us notice the free energy scaling result for the solutions having several tachyon zeroes. Following the arguments of section (10.2), we see that the energy difference between the solution with vanishing quark mass and the solution with $n$ tachyon zeroes scales as

$$
\frac{\Delta \mathcal{E}}{M^{3} N^{2} V_{4}} \sim r_{\mathrm{UV}}^{-4} \exp \left[-\frac{4 K(n+1)}{\sqrt{\lambda_{*}-\lambda_{c}}}\right] \sim r_{\mathrm{UV}}^{-4} \exp \left[-\frac{4 \hat{K}(n+1)}{\sqrt{x_{c}-x}}\right] .
$$

Since we have numerically checked that $\Delta \mathcal{E}$ is positive, this result verifies that the $n=0$ solution has the lowest free energy for $x \rightarrow x_{c}$.

Open Access. This article is distributed under the terms of the Creative Commons Attribution License which permits any use, distribution and reproduction in any medium, provided the original author(s) and source are credited.

\section{References}

[1] G. 't Hooft, A planar diagram theory for strong interactions, Nucl. Phys. B 72 (1974) 461 [INSPIRE].

[2] G. Veneziano, U(1) without instantons, Nucl. Phys. B 159 (1979) 213 [INSPIRE].

[3] T. Banks and A. Zaks, On the phase structure of vector-like gauge theories with massless fermions, Nucl. Phys. B 196 (1982) 189 [INSPIRE].

[4] M.G. Alford, K. Rajagopal and F. Wilczek, QCD at finite baryon density: nucleon droplets and color superconductivity, Phys. Lett. B 422 (1998) 247 [hep-ph/9711395] [INSPIRE].

[5] M.G. Alford, K. Rajagopal and F. Wilczek, Color flavor locking and chiral symmetry breaking in high density QCD, Nucl. Phys. B 537 (1999) 443 [hep-ph/9804403] [InSPIRE].

[6] V. Miransky and K. Yamawaki, Conformal phase transition in gauge theories, Phys. Rev. D 55 (1997) 5051 [Erratum ibid. D 56 (1997) 3768] [hep-th/9611142] [INSPIRE].

[7] D.B. Kaplan, J.-W. Lee, D.T. Son and M.A. Stephanov, Conformality lost, Phys. Rev. D 80 (2009) 125005 [arXiv:0905.4752] [INSPIRE].

[8] J.M. Kosterlitz, The Critical properties of the two-dimensional x y model, J. Phys. C 7 (1974) 1046.

[9] V. Miransky, Dynamics of spontaneous chiral symmetry breaking and continuum limit in quantum electrodynamics, Nuovo Cim. A 90 (1985) 149 [INSPIRE].

[10] K. Jensen, A. Karch, D.T. Son and E.G. Thompson, Holographic Berezinskii-Kosterlitz-Thouless transitions, Phys. Rev. Lett. 105 (2010) 041601 [arXiv: 1002.3159] [INSPIRE].

[11] K. Jensen, More holographic Berezinskii-Kosterlitz-Thouless transitions, Phys. Rev. D 82 (2010) 046005 [arXiv:1006.3066] [INSPIRE].

[12] N. Iqbal, H. Liu, M. Mezei and Q. Si, Quantum phase transitions in holographic models of magnetism and superconductors, Phys. Rev. D 82 (2010) 045002 [arXiv:1003.0010] [INSPIRE]. 
[13] N. Evans, A. Gebauer, K.-Y. Kim and M. Magou, Phase diagram of the D3/D5 system in a magnetic field and a BKT transition, Phys. Lett. B 698 (2011) 91 [arXiv:1003.2694] [INSPIRE].

[14] S.S. Pal, Quantum phase transition in a Dp-Dq system, Phys. Rev. D 82 (2010) 086013 [arXiv: 1006.2444] [INSPIRE].

[15] D. Kutasov, J. Lin and A. Parnachev, Conformal phase transitions at weak and strong coupling, Nucl. Phys. B 858 (2012) 155 [arXiv:1107.2324] [INSPIRE].

[16] N. Iqbal, H. Liu and M. Mezei, Quantum phase transitions in semi-local quantum liquids, arXiv: 1108.0425 [INSPIRE].

[17] N. Iqbal, H. Liu and M. Mezei, Lectures on holographic non-Fermi liquids and quantum phase transitions, arXiv:1110.3814 [INSPIRE].

[18] T. Appelquist, K.D. Lane and U. Mahanta, On the Ladder approximation for spontaneous chiral symmetry breaking, Phys. Rev. Lett. 61 (1988) 1553 [INSPIRE].

[19] A.G. Cohen and H. Georgi, Walking beyond the rainbow, Nucl. Phys. B 314 (1989) 7 [INSPIRE].

[20] E. Gardi and G. Grunberg, The conformal window in QCD and supersymmetric QCD, JHEP 03 (1999) 024 [hep-th/9810192] [INSPIRE].

[21] T. Appelquist, A.G. Cohen and M. Schmaltz, A new constraint on strongly coupled gauge theories, Phys. Rev. D 60 (1999) 045003 [hep-th/9901109] [INSPIRE].

[22] F. Sannino and K. Tuominen, Orientifold theory dynamics and symmetry breaking, Phys. Rev. D 71 (2005) 051901 [hep-ph/0405209] [INSPIRE].

[23] H. Gies and J. Jaeckel, Chiral phase structure of QCD with many flavors, Eur. Phys. J. C 46 (2006) 433 [hep-ph/0507171] [INSPIRE].

[24] T.A. Ryttov and F. Sannino, Supersymmetry inspired QCD $\beta$-function, Phys. Rev. D 78 (2008) 065001 [arXiv:0711.3745] [InSPIRE].

[25] E. Poppitz and M. Ünsal, Conformality or confinement: (IR)relevance of topological excitations, JHEP 09 (2009) 050 [arXiv: 0906.5156] [INSPIRE].

[26] A. Armoni, The conformal window from the worldline formalism, Nucl. Phys. B 826 (2010) 328 [arXiv:0907.4091] [INSPIRE].

[27] M.T. Frandsen, T. Pickup and M. Teper, Delineating the conformal window, Phys. Lett. B 695 (2011) 231 [arXiv:1007.1614] [INSPIRE].

[28] O. Antipin and K. Tuominen, Constraints on conformal windows from holographic duals, Mod. Phys. Lett. A 26 (2011) 2227 [arXiv:0912.0674] [INSPIRE].

[29] T. Appelquist, G.T. Fleming and E.T. Neil, Lattice study of conformal behavior in SU(3) Yang-Mills theories, Phys. Rev. D 79 (2009) 076010 [arXiv:0901.3766] [InSPIRE].

[30] M. Hayakawa, K.-I. Ishikawa, Y. Osaki, S. Takeda, S. Uno and N. Yamada, Running coupling constant of ten-flavor QCD with the Schródinger functional method, Phys. Rev. D 83 (2011) 074509 [arXiv:1011.2577] [InSPIRE].

[31] Z. Fodor et al., Twelve massless flavors and three colors below the conformal window, Phys. Lett. B 703 (2011) 348 [arXiv:1104.3124] [INSPIRE].

[32] B. Holdom, Techniodor, Phys. Lett. B 150 (1985) 301 [INSPIRE]. 
[33] K. Yamawaki, M. Bando and K.-i. Matumoto, Scale invariant technicolor model and a technidilaton, Phys. Rev. Lett. 56 (1986) 1335 [INSPIRE].

[34] T.W. Appelquist, D. Karabali and L. Wijewardhana, Chiral hierarchies and the flavor changing neutral current problem in technicolor, Phys. Rev. Lett. 57 (1986) 957 [INSPIRE].

[35] K.D. Lane, An introduction to technicolor, in Building Blocks of Creation, From Microfermis to Megaparsecs, Proceedings of the 1993 Theoretical Advanced Study Institute in Elementary Particle Physics, Boulder U.S.A. (1993), World Scientific, Singapore (1994), pg. 32 [hep-ph/9401324] [INSPIRE].

[36] C.T. Hill and E.H. Simmons, Strong dynamics and electroweak symmetry breaking, Phys. Rept. 381 (2003) 235 [Erratum ibid. 390 (2004) 553-554] [hep-ph/0203079] [INSPIRE].

[37] F. Sannino, Conformal dynamics for TeV physics and cosmology, Acta Phys. Polon. B 40 (2009) 3533 [arXiv: 0911.0931] [INSPIRE].

[38] B. Holdom, Raising condensates beyond the ladder, Phys. Lett. B 213 (1988) 365 [InSPIRE].

[39] H.S. Fukano and F. Sannino, Conformal window of gauge theories with four-fermion interactions and ideal walking, Phys. Rev. D 82 (2010) 035021 [arXiv:1005.3340] [INSPIRE].

[40] M.E. Peskin and T. Takeuchi, Estimation of oblique electroweak corrections, Phys. Rev. D 46 (1992) 381 [inSPIRE].

[41] F. Sannino, Mass deformed exact S-parameter in conformal theories, Phys. Rev. D 82 (2010) 081701 [arXiv:1006.0207] [inSPIRE].

[42] F. Sannino, Magnetic S-parameter, Phys. Rev. Lett. 105 (2010) 232002 [arXiv:1007.0254] [INSPIRE].

[43] S. Di Chiara, C. Pica and F. Sannino, Flavor dependence of the S-parameter, Phys. Lett. B $\mathbf{7 0 0}$ (2011) 229 [arXiv: 1008.1267] [INSPIRE].

[44] T. Appelquist and F. Sannino, The physical spectrum of conformal $\mathrm{SU}(N)$ gauge theories, Phys. Rev. D 59 (1999) 067702 [hep-ph/9806409] [INSPIRE].

[45] J. Hirn and V. Sanz, A negative S parameter from holographic technicolor, Phys. Rev. Lett. 97 (2006) 121803 [hep-ph/0606086] [INSPIRE].

[46] J. Hirn and V. Sanz, The fifth dimension as an analogue computer for strong interactions at the LHC, JHEP 03 (2007) 100 [hep-ph/0612239] [INSPIRE].

[47] D.K. Hong and H.-U. Yee, Holographic estimate of oblique corrections for technicolor, Phys. Rev. D 74 (2006) 015011 [hep-ph/0602177] [INSPIRE].

[48] M. Piai, Precision electro-weak parameters from $A d S_{5}$, localized kinetic terms and anomalous dimensions, hep-ph/0608241 [INSPIRE].

[49] C.D. Carone, J. Erlich and J.A. Tan, Holographic bosonic technicolor, Phys. Rev. D 75 (2007) 075005 [hep-ph/0612242] [INSPIRE].

[50] M. Fabbrichesi, M. Piai and L. Vecchi, Dynamical electro-weak symmetry breaking from deformed AdS: vector mesons and effective couplings, Phys. Rev. D 78 (2008) 045009 [arXiv: 0804.0124] [INSPIRE].

[51] K. Haba, S. Matsuzaki and K. Yamawaki, S parameter in the holographic walking/conformal technicolor, Prog. Theor. Phys. 120 (2008) 691 [arXiv:0804.3668] [INSPIRE]. 
[52] J. Hirn, A. Martin and V. Sanz, Describing viable technicolor scenarios, Phys. Rev. D 78 (2008) 075026 [arXiv:0807.2465] [INSPIRE].

[53] D.D. Dietrich and C. Kouvaris, Constraining vectors and axial-vectors in walking technicolour by a holographic principle, Phys. Rev. D 78 (2008) 055005 [arXiv:0805.1503] [INSPIRE].

[54] D.D. Dietrich and C. Kouvaris, Generalised bottom-up holography and walking technicolour, Phys. Rev. D 79 (2009) 075004 [arXiv:0809.1324] [INSPIRE].

[55] D.D. Dietrich, M. Järvinen and C. Kouvaris, Mixing in the axial sector in bottom-up holography for walking technicolour, JHEP 07 (2010) 023 [arXiv:0908.4357] [INSPIRE].

[56] J. Erlich, E. Katz, D.T. Son and M.A. Stephanov, QCD and a holographic model of hadrons, Phys. Rev. Lett. 95 (2005) 261602 [hep-ph/0501128] [INSPIRE].

[57] L. Da Rold and A. Pomarol, Chiral symmetry breaking from five dimensional spaces, Nucl. Phys. B 721 (2005) 79 [hep-ph/0501218] [INSPIRE].

[58] C. Núñez, I. Papadimitriou and M. Piai, Walking dynamics from string duals, Int. J. Mod. Phys. A 25 (2010) 2837 [arXiv:0812.3655] [InSPIRE].

[59] S. Kumar, D. Mateos, A. Paredes and M. Piai, Towards holographic walking from $N=4$ super Yang-Mills, JHEP 05 (2011) 008 [arXiv: 1012.4678] [INSPIRE].

[60] L. Anguelova, P. Suranyi and L. Wijewardhana, Holographic walking technicolor from D-branes, Nucl. Phys. B 852 (2011) 39 [arXiv:1105.4185] [INSPIRE].

[61] N. Seiberg, Exact results on the space of vacua of four-dimensional SUSY gauge theories, Phys. Rev. D 49 (1994) 6857 [hep-th/9402044] [INSPIRE].

[62] N. Seiberg, Electric-magnetic duality in supersymmetric nonAbelian gauge theories, Nucl. Phys. B 435 (1995) 129 [hep-th/9411149] [InSPIRE].

[63] K.A. Intriligator and N. Seiberg, Lectures on supersymmetric gauge theories and electric-magnetic duality, Nucl. Phys. Proc. Suppl. 45BC (1996) 1 [hep-th/9509066] [INSPIRE].

[64] M.J. Strassler, Millenial messages for QCD from the superworld and from the string, in At the frontier of particle physics. Vol. 3: Handbook of QCD, M. Shifman eds., World Scientific, Singapore (2003), pg. 1859. hep-th/0309140 [INSPIRE].

[65] Z. Komargodski, Vector mesons and an interpretation of Seiberg duality, JHEP 02 (2011) 019 [arXiv: 1010.4105] [INSPIRE].

[66] I.R. Klebanov and J.M. Maldacena, Superconformal gauge theories and non-critical superstrings, Int. J. Mod. Phys. A 19 (2004) 5003 [hep-th/0409133] [INSPIRE].

[67] F. Bigazzi, R. Casero, A. Cotrone, E. Kiritsis and A. Paredes, Non-critical holography and four-dimensional CFT's with fundamentals, JHEP 10 (2005) 012 [hep-th/0505140] [INSPIRE].

[68] E. Kiritsis, C. Kounnas and D. Lüst, A large class of new gravitational and axionic backgrounds for four-dimensional superstrings, Int. J. Mod. Phys. A 9 (1994) 1361 [hep-th/9308124] [INSPIRE].

[69] A. Barranco, E. Pallante and J.G. Russo, $N=1$ SQCD-like theories with $N_{f}$ massive flavors from AdS/CFT and $\beta$-functions, JHEP 09 (2011) 086 [arXiv:1107.4002] [INSPIRE]. 
[70] J. Polchinski and M.J. Strassler, Hard scattering and gauge/string duality, Phys. Rev. Lett. 88 (2002) 031601 [hep-th/0109174] [INSPIRE].

[71] U. Gürsoy and E. Kiritsis, Exploring improved holographic theories for QCD: Part I, JHEP 02 (2008) 032 [arXiv:0707.1324] [INSPIRE].

[72] U. Gürsoy, E. Kiritsis and F. Nitti, Exploring improved holographic theories for QCD: Part II, JHEP 02 (2008) 019 [arXiv:0707.1349] [InSPIRE].

[73] E. Kiritsis, Dissecting the string theory dual of QCD, Fortsch. Phys. 57 (2009) 396 [arXiv:0901.1772] [INSPIRE].

[74] U. Gürsoy, E. Kiritsis, L. Mazzanti, G. Michalogiorgakis and F. Nitti, Improved holographic QCD, Lect. Notes Phys. 828 (2011) 79 [arXiv:1006.5461] [INSPIRE].

[75] U. Gürsoy, E. Kiritsis, L. Mazzanti and F. Nitti, Deconfinement and gluon plasma dynamics in improved holographic QCD, Phys. Rev. Lett. 101 (2008) 181601 [arXiv:0804.0899] [INSPIRE].

[76] U. Gürsoy, E. Kiritsis, L. Mazzanti and F. Nitti, Holography and thermodynamics of 5D dilaton-gravity, JHEP 05 (2009) 033 [arXiv:0812.0792] [INSPIRE].

[77] U. Gürsoy, E. Kiritsis, L. Mazzanti and F. Nitti, Improved holographic Yang-Mills at finite temperature: comparison with data, Nucl. Phys. B 820 (2009) 148 [arXiv:0903.2859] [INSPIRE].

[78] M. Panero, Thermodynamics of the QCD plasma and the large-N limit, Phys. Rev. Lett. 103 (2009) 232001 [arXiv:0907.3719] [INSPIRE].

[79] J. Alanen, K. Kajantie and V. Suur-Uski, Spatial string tension of finite temperature QCD matter in gauge/gravity duality, Phys. Rev. D 80 (2009) 075017 [arXiv:0905.2032] [INSPIRE].

[80] J. Alanen, K. Kajantie and V. Suur-Uski, A gauge/gravity duality model for gauge theory thermodynamics, Phys. Rev. D 80 (2009) 126008 [arXiv:0911.2114] [INSPIRE].

[81] S.S. Gubser and A. Nellore, Mimicking the QCD equation of state with a dual black hole, Phys. Rev. D 78 (2008) 086007 [arXiv:0804.0434] [INSPIRE].

[82] O. DeWolfe, S.S. Gubser and C. Rosen, A holographic critical point, Phys. Rev. D 83 (2011) 086005 [arXiv:1012.1864] [INSPIRE].

[83] U. Gürsoy, E. Kiritsis, G. Michalogiorgakis and F. Nitti, Thermal transport and drag force in improved holographic QCD, JHEP 12 (2009) 056 [arXiv:0906.1890] [INSPIRE].

[84] U. Gürsoy, E. Kiritsis, L. Mazzanti and F. Nitti, Langevin diffusion of heavy quarks in non-conformal holographic backgrounds, JHEP 12 (2010) 088 [arXiv:1006.3261] [INSPIRE].

[85] E. Kiritsis, L. Mazzanti and F. Nitti, Dressed spectral densities for heavy quark diffusion in holographic plasmas, arXiv:1111.1008 [INSPIRE].

[86] M. Järvinen and F. Sannino, Holographic conformal window - a bottom up approach, JHEP 05 (2010) 041 [arXiv:0911.2462] [InSPIRE].

[87] J. Alanen and K. Kajantie, Thermodynamics of a field theory with infrared fixed point from gauge/gravity duality, Phys. Rev. D 81 (2010) 046003 [arXiv: 0912.4128] [INSPIRE].

[88] J. Alanen, K. Kajantie and K. Tuominen, Thermodynamics of quasi conformal theories from gauge/gravity duality, Phys. Rev. D 82 (2010) 055024 [arXiv:1003.5499] [INSPIRE]. 
[89] J. Alanen, T. Alho, K. Kajantie and K. Tuominen, Mass spectrum and thermodynamics of quasi-conformal gauge theories from gauge/gravity duality, Phys. Rev. D 84 (2011) 086007 [arXiv:1107.3362] [INSPIRE].

[90] A. Karch, E. Katz, D.T. Son and M.A. Stephanov, Linear confinement and AdS/QCD, Phys. Rev. D 74 (2006) 015005 [hep-ph/0602229] [INSPIRE].

[91] R. Casero, E. Kiritsis and A. Paredes, Chiral symmetry breaking as open string tachyon condensation, Nucl. Phys. B 787 (2007) 98 [hep-th/0702155] [INSPIRE].

[92] A. Sen, Tachyon dynamics in open string theory, Int. J. Mod. Phys. A 20 (2005) 5513 [hep-th/0410103] [INSPIRE].

[93] A. Sen, Dirac- Born-Infeld action on the tachyon kink and vortex, Phys. Rev. D 68 (2003) 066008 [hep-th/0303057] [INSPIRE].

[94] C. Kennedy and A. Wilkins, Ramond-Ramond couplings on brane-antibrane systems, Phys. Lett. B 464 (1999) 206 [hep-th/9905195] [INSPIRE].

[95] P. Kraus and F. Larsen, Boundary string field theory of the DD system, Phys. Rev. D 63 (2001) 106004 [hep-th/0012198] [INSPIRE].

[96] T. Takayanagi, S. Terashima and T. Uesugi, Brane-antibrane action from boundary string field theory, JHEP 03 (2001) 019 [hep-th/0012210] [INSPIRE].

[97] O. Bergman, S. Seki and J. Sonnenschein, Quark mass and condensate in HQCD, JHEP 12 (2007) 037 [arXiv:0708.2839] [InSPIRE].

[98] A. Dhar and P. Nag, Sakai-Sugimoto model, tachyon condensation and chiral symmetry breaking, JHEP 01 (2008) 055 [arXiv:0708.3233] [INSPIRE].

[99] A. Dhar and P. Nag, Tachyon condensation and quark mass in modified Sakai-Sugimoto model, Phys. Rev. D 78 (2008) 066021 [arXiv:0804.4807] [INSPIRE].

[100] N. Jokela, M. Järvinen and S. Nowling, Winding effects on brane/anti-brane pairs, JHEP 07 (2009) 085 [arXiv:0901.0281] [INSPIRE].

[101] I. Iatrakis, E. Kiritsis and A. Paredes, An AdS/QCD model from Sen's tachyon action, Phys. Rev. D 81 (2010) 115004 [arXiv:1003.2377] [InSPIRE].

[102] I. Iatrakis, E. Kiritsis and A. Paredes, An AdS/QCD model from tachyon condensation: II, JHEP 11 (2010) 123 [arXiv:1010.1364] [INSPIRE].

[103] S. Kuperstein and J. Sonnenschein, Non-critical, near extremal AdS $S_{6}$ background as a holographic laboratory of four dimensional YM theory, JHEP 11 (2004) 026 [hep-th/0411009] [INSPIRE].

[104] D. Arean, I. Iatrakis, M. Järvinen and E. Kiritsis, Meson spectra in V-QCD, work in progress.

[105] K. Yamawaki, M. Bando and K.-i. Matumoto, Scale invariant technicolor model and a technidilaton, Phys. Rev. Lett. 56 (1986) 1335 [INSPIRE].

[106] T. Alho, M. Järvinen, K. Kajantie, E. Kiritsis and K. Tuominen, Finite temperature phase diagram of $V$-QCD, work in progress.

[107] C. Núñez, A. Paredes and A.V. Ramallo, Unquenched flavor in the gauge/gravity correspondence, Adv. High Energy Phys. 2010 (2010) 196714 [arXiv: 1002.1088] [INSPIRE]. 
[108] C. Charmousis, B. Gouteraux, B. Kim, E. Kiritsis and R. Meyer, Effective holographic theories for low-temperature condensed matter systems, JHEP 11 (2010) 151 [arXiv: 1005.4690] [INSPIRE].

[109] B. Gouteraux and E. Kiritsis, Generalized holographic quantum criticality at finite density, JHEP 12 (2011) 036 [arXiv:1107.2116] [INSPIRE].

[110] E. Kiritsis and F. Nitti, On massless $4 D$ gravitons from asymptotically Ad $S_{5}$ space-times, Nucl. Phys. B 772 (2007) 67 [hep-th/0611344] [INSPIRE].

[111] C.J. Morningstar and M.J. Peardon, The glueball spectrum from an anisotropic lattice study, Phys. Rev. D 60 (1999) 034509 [hep-lat/9901004] [INSPIRE].

[112] Y. Chen et al., Glueball spectrum and matrix elements on anisotropic lattices, Phys. Rev. D 73 (2006) 014516 [hep-lat/0510074] [INSPIRE].

[113] I. Papadimitriou, Holographic renormalization of general dilaton-axion gravity, JHEP 08 (2011) 119 [arXiv:1106.4826] [INSPIRE].

[114] J. Erdmenger, A. Gorsky, P.N. Kopnin, A. Krikun and A.V. Zayakin, Low-energy theorems from holography, JHEP 03 (2011) 044 [arXiv:1101.1586] [INSPIRE].

[115] P. Breitenlohner and D.Z. Freedman, Positive energy in anti-de Sitter backgrounds and gauged extended supergravity, Phys. Lett. B 115 (1982) 197 [INSPIRE].

[116] T. Appelquist, K.D. Lane and U. Mahanta, On the ladder approximation for spontaneous chiral symmetry breaking, Phys. Rev. Lett. 61 (1988) 1553 [INSPIRE].

[117] D.D. Dietrich and F. Sannino, Conformal window of $\mathrm{SU}(N)$ gauge theories with fermions in higher dimensional representations, Phys. Rev. D 75 (2007) 085018 [hep-ph/0611341] [INSPIRE].

[118] C. Pica and F. Sannino, $\beta$-function and anomalous dimensions, Phys. Rev. D 83 (2011) 116001 [arXiv:1011.3832] [INSPIRE].

[119] D.D. Dietrich, A mass-dependent $\beta$-function, Phys. Rev. D 80 (2009) 065032 [arXiv:0908.1364] [INSPIRE].

[120] D.D. Dietrich, Quasiconformality and mass, Phys. Rev. D 82 (2010) 065007 [arXiv: 1005.1324] [INSPIRE].

[121] S.S. Gubser, Curvature singularities: the good, the bad and the naked, Adv. Theor. Math. Phys. 4 (2000) 679 [hep-th/0002160] [INSPIRE]. 